\title{
Characterization of inner mitochondrial membrane architecture
}

\author{
Dissertation \\ for the award of the degree \\ "Doctor rerum naturalium" (Dr. rer. nat.) \\ Division of Mathematics and Natural Sciences \\ of the Georg-August-Universität Göttingen
}

submitted by

Mariam Barbot

from

Tbilisi, Georgia

Göttingen, 2016 



\title{
Members of the Thesis Committee:
}

\author{
Prof. Dr. Michael Meinecke (first reviewer)
}

Department of Cellular Biochemistry, University Medical Center Göttingen

Prof. Dr. Stefan Jakobs (second reviewer)

Department of NanoBiophotonics, Max Planck Institute for Biophysical Chemistry, Göttingen

Prof. Dr. Tobias Moser

Institute of Auditory Neuroscience and InnerEarLab, University Medical Center Göttingen

\section{Additional Reviewers:}

Prof. Dr. Ralph Kehlenbach

Department of Molecular Biology, University Medical Center Göttingen

Prof. Dr. Dörthe Katschinski

Department Cardiovascular Physiology, University Medical Center Göttingen

Prof. Dr. Claudia Steinem

Institute of Organic and Biomolecular Chemistry, University of Göttingen

Date of the oral examination: 2.06.2016 



\section{Affidavit}

I hereby declare that this thesis has been written independently and with no other sources and aids than quoted.

Mariam Barbot

Göttingen, 30 March 2016 

- to my parents -

- to Yann - 



\section{List of Publications}

A part of this thesis has been previously published or is in revision in the following articles:

Barbot, M., Jans, D. C., Schulz, C., Denkert, N., Kroppen, B., Hoppert, M., Jakobs, S., and Meinecke, M. (2015). Mic10 oligomerizes to bend mitochondrial inner membranes at crisate junctions. Cell Metab, 21(5):756-763.

Barbot, M. and Meinecke, M. (2016). Reconstitution of mitochondrial inner membrane remodeling. In revision.

Gleisner M., Mey, I., Barbot, M., Dreker, C., Meinecke, M., and Steinem, C. (2014). Driving a planar model system into the 3(rd) dimension: generation and control of curved pore-spanning membrane arrays. Soft Matter., 10(33):6228-6236. ^ $^{*}$

${ }^{*}$ results of this publication are not discussed in this thesis 



\section{Abstract}

The inner membrane of mitochondria is extensively folded and displays a complex architecture. Cristae junctions are highly curved tubular openings that separate the cristae membrane from the inner boundary membrane. They play a central role in many vital cellular processes (e.g. apoptosis) by compartmentalizing the inner membrane into morphologically and functionally distinct regions. The mitochondrial contact site and cristae organizing system (MICOS) is a conserved multi-subunit protein complex, which is found to be enriched at cristae junctions. The complex is believed to be necessary for maintaining the physiological cristae membrane morphology by stabilizing cristae junctions.

In this thesis, the role of the MICOS core subunit, Mic10, in the formation and maintenance of cristae junctions was investigated. An in vitro bottom up approach, in combination with in organello studies, was used to address the direct role and molecular mechanism by which Mic10 sculpts the inner mitochondrial membrane at cristae junctions. The reconstitution of recombinantly expressed and purified Saccharomyces cerevisiae Mic10 into artificial model membranes led to drastic membrane morphology changes. Membrane topology studies revealed that Mic10 contains two transmembrane domains that span the inner mitochondrial membrane in a hairpin-like structure. The ability of Mic10 to sculpt membranes depends on the protein's homooligomerization through highly conserved glycine-rich motifs present in both transmembrane domains. The disruption of Mic10 oligomerization by mutating glycine residues failed to induce curvature in model membranes and resulted in abnormal inner membrane architecture when expressed in yeast cells.

Collectively, these findings demonstrate that membrane sculpting by Mic10 is essential for both cristae junction formation and stabilization. 



\section{Table of Contents}

Abstract ix

List of Abbreviations $\quad$ xv

1 Introduction 1

1.1 Characteristic shapes of organellar membranes . . . . . . . . . 1

1.2 Role of mitochondria in biological processes . . . . . . . . . . 2

1.3 Mitochondria are dynamic organelles . . . . . . . . . . . . 3

1.4 Mitochondrial membrane composition and ultrastructure . . . . . . . . 4

1.4.1 Proteins underlying the inner mitochondrial membrane morphol-

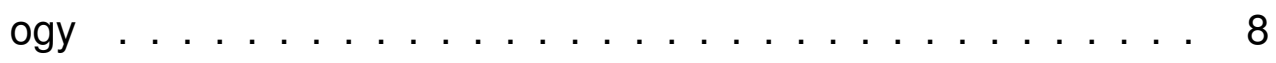

1.4 .1 .1 ATP synthase $\ldots \ldots \ldots \ldots \ldots \ldots$

1.4 .1 .2 Mgm1/OPA1 $\ldots \ldots \ldots \ldots \ldots$

1.4 .1 .3 MICOS complex . . . . . . . . . . . . 13

$\begin{array}{lll}2 \text { Aim of this Work } & 21\end{array}$

3 Materials and Methods 23

3.1 Materials . . . . . . . . . . . . . . . . . 23

3.1 .1 Chemicals and lipids $\ldots \ldots \ldots \ldots$

3.1 .2 Antibodies. . . . . . . . . . . . . . . . . 26

3.1 .3 Microorganisms . . . . . . . . . . . . . . . 27

3.1 .4 Plasmids . . . . . . . . . . . . . . . . . . . . . . . 29

3.1 .5 Instruments and software $\ldots \ldots \ldots . \ldots . \ldots 30$

3.2 Methods . . . . . . . . . . . . . . . . . . . . . . 32

$3.2 .1 \quad$ Cultivation and application of biological material $\ldots \ldots . .32$

3.2 .1 .1 E.coli growth . . . . . . . . . . . . . . . . . 32

3.2.1.2 Preparation and transformation of chemically competent E.coli cells . . . . . . . . . . . . . . . . . 33 
3.2.1.3 Preparation of E.coli whole cell lysate . . . . . . . . 33

3.2 .1 .4 S. cerevisiae growth . . . . . . . . . . . . . . . . . . . 34

3.2.1.5 Preparation and transformation of chemically competent $S$. cerevisiae cells . . . . . . . . . . . . . . . 34

3.2.1.6 Isolation of mitochondria form S. cerevisiae cells . . . . 35

3.2 .2 Molecular biology . . . . . . . . . . . . . . . . . . . 36

3.2.2.1 Plasmid DNA isolation from E.coli cells . . . . . . . . 36

3.2.2.2 Polymerase chain reaction (PCR) . . . . . . . . . 36

3.2 .2 .3 Cloning . . . . . . . . . . . . . . . . . 37

3.2 .2 .4 DNA sequencing. . . . . . . . . . . . . . . . . 37

3.2 .3 Protein biochemistry . . . . . . . . . . . . . . . . . . . 37

3.2 .3 .1 SDS-PAGE . . . . . . . . . . . . . . . . . . . 37

3.2 .3 .2 BN-PAGE . . . . . . . . . . . . . . . . . . . . 38

3.2.3.3 Coomassie Brilliant Blue staining . . . . . . . . 38

3.2 .3 .4 Western blot . . . . . . . . . . . . . . . . . . . . . . . 39

3.2 .3 .5 Immunodetection . . . . . . . . . . . . . . . . 39

3.2.3.6 Recombinant protein affinity-tag purification from E.coli 40

3.2 .3 .7 Size-exclusion chromatography $\ldots \ldots \ldots \ldots$. . . . 41

3.2 .3 .8 Protein precipitation by TCA . . . . . . . . . . . . . 42

3.2.3.9 Protein refolding in various mild detergents . . . . . . . 42

3.2 .3 .10 Protein labeling . . . . . . . . . . . . . . 43

3.2.3.11 Protein membrane topology mapping . . . . . . . . 4 43

3.2.3.12 Mic10 co-immunoprecipitation . . . . . . . . . . . . 44

3.2 .4 Handling of artificial lipid membranes . . . . . . . . . . . 44

3.2 .4 .1 Liposome preparation . . . . . . . . . . . . . . . . . 44

3.2 .4 .2 Proteo-liposome preparation . . . . . . . . . . . . 45

3.2 .4 .3 Flotation assay . . . . . . . . . . . . . . . . . . 46

3.2 .4 .4 Sodium carbonate extraction . . . . . . . . . . . . . 46

3.2 .4 .5 DLS measuments . . . . . . . . . . . . . . . 46

3.2 .4 .6 Preparation of GUVs . . . . . . . . . . . . . 46

3.2.4.7 FRET measurements . . . . . . . . . . . . . . . 47

3.2 .5 Visualization techniques . . . . . . . . . . . . . . . . 47

$3.2 .5 .1 \quad$ EM microscopy of LUVs. . . . . . . . . . . . . . . . 47

3.2.5.2 Electron microscopy of yeast cells . . . . . . . . . . 48 
4.1 Mic10 induces high degrees of membrane curvature in model membranes . . . . . . . . . . . . . . . . . . . 49

4.1 .1 Purification of recombinantly expressed Mic10 . . . . . . . . . . 49

4.1.2 Mic10 can be successfully integrated into model membranes . . 52

4.1 .3 Mic10 changes the morphology of model membranes . . . . . . 54

4.2 Mic10 membrane topology mapping . . . . . . . . . . . . . . . . . 59

4.2 .1 Trials to obtain Mic10 structure . . . . . . . . . . . . . . . . . . . 59

4.2.2 Mic10 spans the mitochondrial inner membrane in a hairpin topology . . . . . . . . . . . . . . . . 6 61

4.3 Mic10 forms homo-oligomers through glycine-rich motifs . . . . . . . . . 64

4.3 .1 Mic10 forms homo-oligomers . . . . . . . . . . . . . . . . . 64

4.3.2 Mic10 molecules are in close proximity in model membranes . . 65

4.3.3 Glycine-rich motifs are important for Mic10 oligomerization . . . 66

4.4 Oligomerization of Mic10 is a prerequisite for membrane bending in vitro 68

4.5 Mic10 oligomerization is essential for normal cristae junction formation in vivo . . . . . . . . . . . . . . . . . . . . . 69

4.5.1 Mic10 oligomerization disruption is not lethal . . . . . . . . . . 72

5 Discussion 73

5.1 Mic10 tubulates round liposomes . . . . . . . . . . . . . . . . . . 73

5.2 Mic10 membrane curving mechanism . . . . . . . . . . . . . . . . 77

5.3 Possible role of other MICOS subunits in cristae sculpting . . . . . . . . 79

5.4 Model of cristae membrane reconstitution . . . . . . . . . . . . . 80

6 Summary and Conclusion $\quad 85$

\begin{tabular}{|lr}
\hline List of Figures & 87
\end{tabular}

\begin{tabular}{ll}
\hline List of Tables & 89
\end{tabular}

\begin{tabular}{ll}
\hline References & 91
\end{tabular}

\begin{tabular}{ll}
\hline Acknowledgments & 109
\end{tabular} 



\section{List of Abbreviations}

${ }^{\circ} \mathrm{C} \quad$... Degree celsius

ADP ... Adenosindiphosphate

APOO ... Apolipoprotein O

APOOL ... Apolipoprotein O-like

ATP ... Adenosintriphosphate

BN-PAGE ... Blue native polyacrylamid gel electrophoresis

BSA ... Bovine serum albumine

$\mathrm{CHCH} \quad \ldots$ Coiled-coil helix coiled-coil helix

CJ ... Cristae junctions

CL ... Cardiolipin

DDM $\ldots$ n-Dodecyl $\beta$-D-maltoside

DLS ... Dynamic light scattering

DM $\ldots$ n-Decyl- $\beta$-D-maltopyranoside

DMSO ... Dimethylsulfoxide

DMPC ... 1,2-dimyristoyl-sn-glycero-3-phosphocholine

Dnase I ... Deoxyribonuclease I

DTT ... Dithiothreitol

ECL ... Enhanced chemiluminescence 
EDTA ... Ethylenediaminetetraacetic acid

EM ... Electron microscope or electron microscopy

ER ... Endoplasmatic reticulum

FRET ... Förster (fluorescence) resonance energy transfer

g ... Gram

GST ... Glutathione S-transferase

GuHCl ... Guanidin hydrochloride

GUV ... Giant unilamellar vesicle

h ... Hour

HEPES ... 4-(2-hydroxyethyl)-1-piperazineethanesulfonic acid

HRP ... Horseradish peroxidase

IBM ... Inner boundary membrane

IgG $\quad$... Immunoglobulin $G$

IM ... Inner (mitochondrial) membrane

IMS ... Intermembrane space

IPTG ... Isopropyl $\beta$-D-1-thiogalactopyranoside

ITO ... Indium tin oxide

L ... Liter

LB ... Lysogeny broth

LUV ... Large unilamellar vesicle

$\mathrm{mA}$... Milliampere

Mega 9 ... Acyl-N-methylglucamine 9 
MES ... 2-(N-morpholino)ethanesulfonic acid

mg ... Milligram

Mgm1 ... Mitochondrial genome maintenance

MIA ... Mitochondrial IMS import and assembly machinery

MICOS ... Mitochondrial contact site and cristae organizing system

$\min$... Minute

$\mathrm{mL} \quad$... Milliliter

$\mathrm{mM} \quad$... Millimolar

MOPS ... 3-(N-morpholino)propanesulfonic acid

MPa ... Megapascal

ng ... Nanogram

nm ... Nanometer

NMR ... Nuclear magnetic resonance

OD ... Optical density

OM ... Outer (mitochondrial) membrane

OPA1 ... Optic atrophy 1

PA ... Phosphatidic acid

PC ... Phosphatidylcholine

PCR ... Polymerase chain reaction

PE ... Phosphatidylethanolamine

PEG ... Polyethylene glycol

PI ... Phosphatidylinositol 
PK ... Proteinase $\mathrm{K}$

PMSF ... Phenylmethylsulfonyl fluoride

PS ... Phosphatidylserin

psi ... Pound-force per square inch

PVDF ... Polyvinylidendifluoride membrane

Rhodamine PE ... L- $\alpha$-PE-N-(lissamine rhodamine B sulfonyl) (Ammonium Salt)

rpm ... Rounds per minute

SAM ... Sorting and assembly machinery

SD ... Selective glucose

SDS-PAGE ... Sodium dodecyl sulfate polyacrylamide gel electrophoresis

SEM ... Sucrose, EDTA, MOPS

SG $\quad .$. Selective glycerol

SLac ... Selective lactate

SUV ... Small unilamellar vesicle

TBS ... Tris-buffered saline

TBS-T ... Tris-buffered saline with Tween

TCA ... Trichloroacetic acid

TEMED ... Tetramethylethylenediamine

TIM ... Translocase of the inner membrane

TOM ... Translocase of the outer membrane

$\mu \mathrm{L} \quad \ldots$ Microliter

Ugo1 ... Mitochondrial fusion and transport protein 
V ... Volt

VDAC ... Voltage-dependent anion channel

w/w ... Wet weight

WT ... Wildtype

xg ... Times gravitation

YPAD ... Yeast extract, peptone, adenin hemisulfate, glucose 



\section{Introduction}

\subsection{Characteristic shapes of organellar membranes}

Cells are the basic biological units of living organisms. They are enclosed and protected from the extracellular environment by membranes. Numerous important biochemical processes such as lipid metabolism occur on biological membranes. In addition, gradients are generated across the membranes, for example, proton gradient to produce cellular ATP.

To increase the efficiency of these processes, eukaryotic cells established organelles thus increasing the membrane surface and creating micro-environments to spatially and temporally separate different metabolic activities. The membranebound organelles have characteristic shapes. For example, peroxisomes and lysosomes are rather spherical, whereas endoplasmic reticulum and Golgi apparatus form more complex networks of sheet-like and tubular membrane structures and mitochondria appear as interconnected tubular networks (Figure 1.1). Among these organelles, mitochondria are probably the most structurally diverse and complex, as they possess two membranes with a highly convoluted inner membrane. In addition, the shape of cellular membranes can undergo changes during processes such as endocytosis, where highly curved vesicles can bud from the essentially flat plasma membrane (Figure 1.1). Generation of these peculiar membrane shapes characterized by different degrees of membrane curvature is challenging for eukaryotic cell, as it is an energetically demanding process. Different membrane proteins provide the energy needed to generate and sustain this curvature (Zimmerberg and Kozlov, 2006; Shibata et al., 2009).

Here, the mechanism of mitochondrial inner membrane morphology generation and maintenance will be discussed. As most work in this field has concerned Saccharomyces cerevisiae, I will focus mainly on the processes occurring in this organism, unless otherwise indicated. 


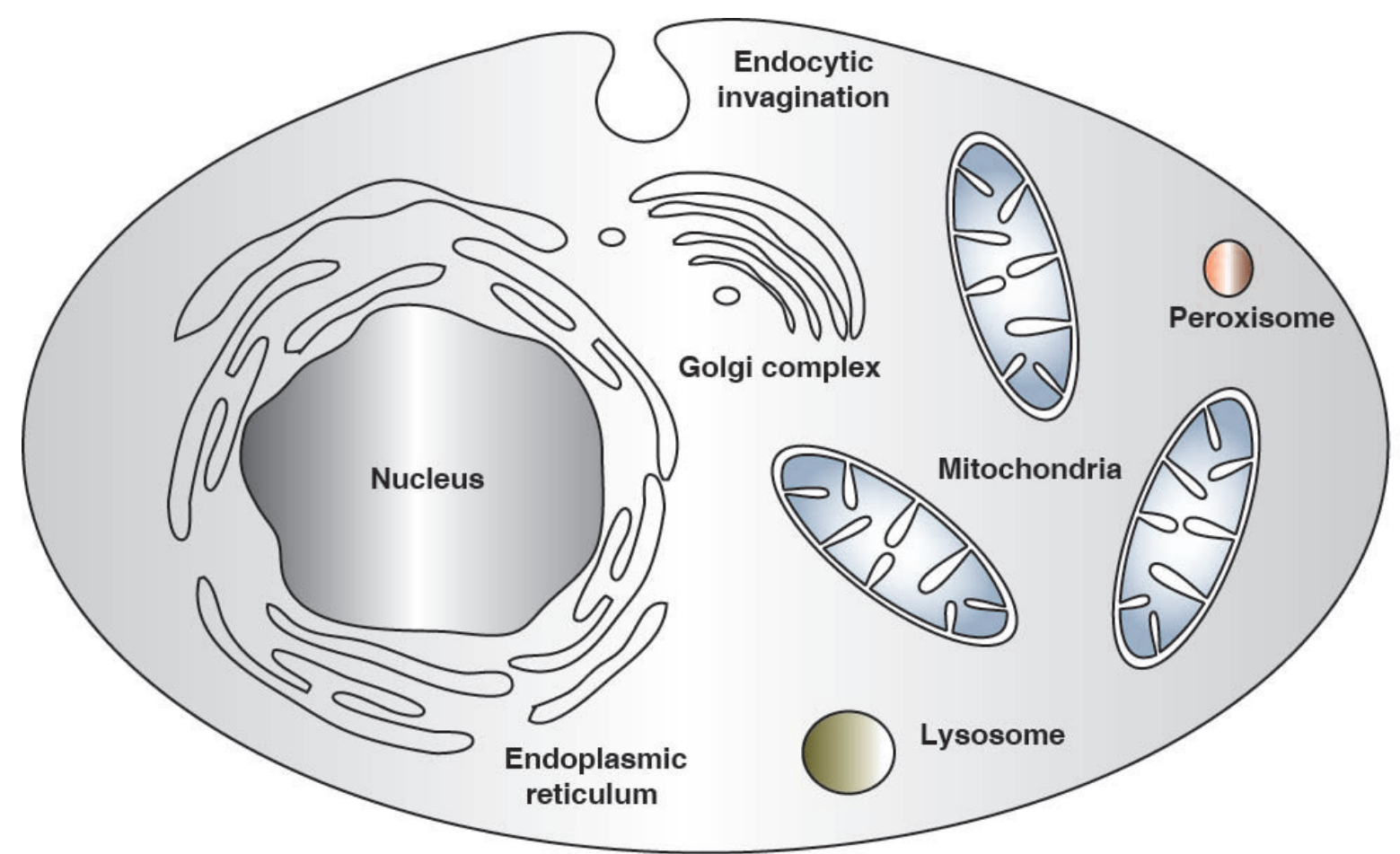

Figure 1.1: The plasma membrane and membrane bound organelles of eukaryotic cell display different morphologies. The plasma membrane encloses the cell and depicts an endocytic invagination. Some organelles in the cytosol, such as lysosomes and peroxisomes, have spherical structures. In contrast, the endoplasmatic reticulum, the golgi apparatus and mitochondria exhibit complex morphologies.

\subsection{Role of mitochondria in biological processes}

Mitochondria are essential organelles in eukaryotic cells. They are well-known for their central role in energy metabolism and ATP production by oxidative phosphorylation.

In addition, mitochondria host many other important cellular processes. The assembly of iron-sulphur clusters occurs in mitochondria, indicating the organelle's fundamental importance in organisms that do not depend on respiration (Lill et al., 2012). Mitochondria are also involved in other metabolic pathways, including the citric acid cycle, urea cycle, $\beta$-oxidation of fatty acids and the biosynthesis of haem and specific phospholipids (Bartlett and Eaton, 2004). Moreover, mitochondria play an 
important role in calcium homeostasis and are a key location of apoptosis (Hardwick and Cheng, 2004; Cheng et al., 2008; Rizzuto et al., 2012)

Mitochondria are semi-autonomous organelles and contain their own genome. However, most of their previously held genetic information was transferred to the nucleus during evolution (Daley et al., 2002; Dyall et al., 2004; Dolezal et al., 2006). In order to fulfill their role in biological processes the mitochondrion relies on imported nuclear-encoded proteins, which is achieved by protein translocases present in both mitochondrial membranes (Neupert and Herrmann, 2007; Dudek et al., 2013; Bohnert et al., 2015a).

\subsection{Mitochondria are dynamic organelles}

Mitochondria have several attributes that would allow them to be described as dynamic organelles. They can freely distribute throughout a cell and enrich at sites where energy demand is high. This is achieved by molecular motors which provide a "vehicle" for the movement of the organelle along cytoskeletal fibers. These movements of mitochondria must be orchestrated with changes in their morphology.

In fact, mitochondria continuously undergo structural changes and can adopt a variety of morphologies, from spheres or short rods to long, tubular interconnected networks. These structural metamorphoses are controlled by the tightly balanced antagonistic interplay of fusion and fission processes (collectively called mitochondrial dynamics) and are in concert with the physiological demands of cells (Westermann, 2010; Labbe et al., 2014). Cells with higher rates of fusion activity relative to fission have a low number of mitochondria, with a tubular, interconnected morphology. These morphologies with their underlying processes play a key role in inheritance and maintenance of mitochondrial DNA and allow efficient energy distribution during energetically demanding processes by mixing and unifying the mitochondrial compartments. Conversely, cells with higher rates of fission have more mitochondria but these are more fragmented, mostly displaying sphere- or rod-like morphologies. Fission processes serve different functions, from playing a role in intrinsic apoptosis to eliminating damaged mitochondria by mitophagy.

In addition to variations in the overall shape, mitochondria display internal structures which are also dynamic and complex. These internal morphologies, which are 
described in detail below also appear to be intimately linked to the bioenergetics of cells and are tightly controlled by proteins.

\subsection{Mitochondrial membrane composition and ultrastructure}

Because of their endosymbiotic origin, mitochondria possess two membranes, the outer membrane (OM) and the inner membrane (IM). These membranes are structurally distinct, which probably gave rise to their functional divergence (Figure 1.2). Moreover, the $\mathrm{OM}$ and the IM are distinct in protein and lipid composition (Zinser et al., 1991).

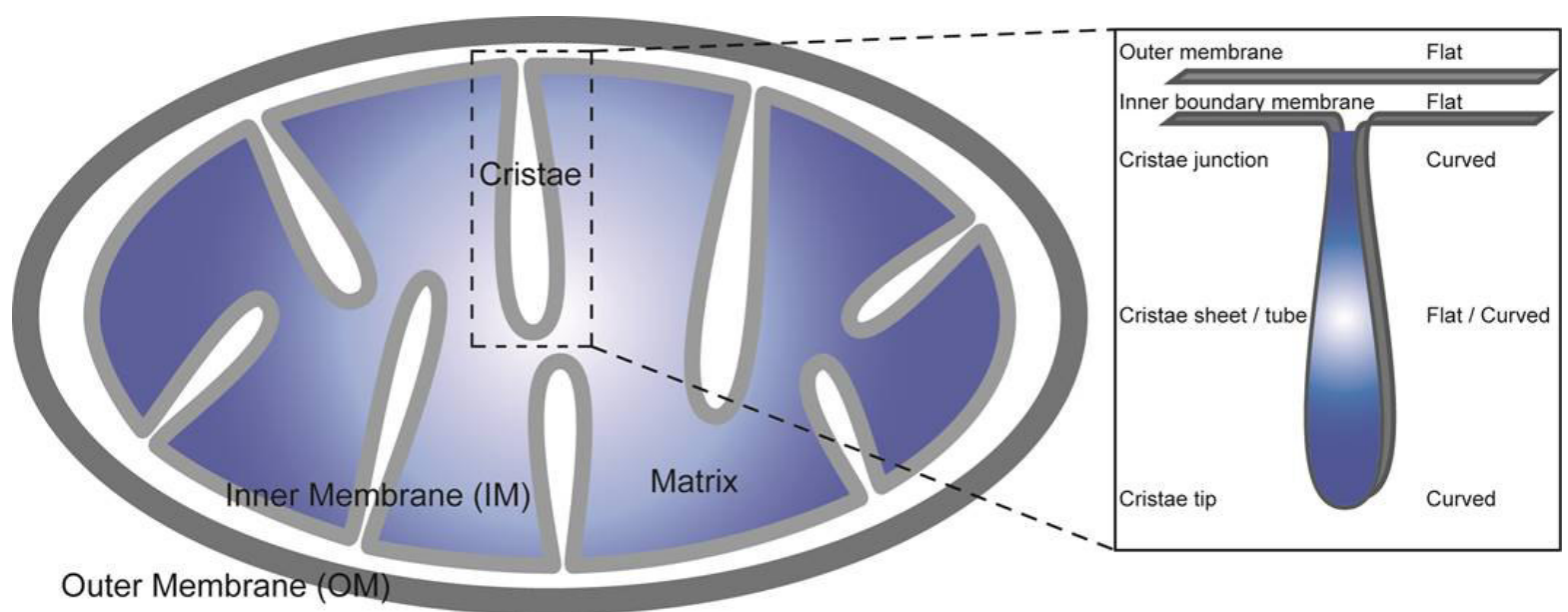

Figure 1.2: Schematic representation of mitochondrial subcompartmentalization and ultrastructure. The mitochondrion is enclosed by two morphologically distinct membranes. The outer membrane physically divides the intermembrane space and the inner membrane from the cytosol. The inner membrane, which encompasses the mitochondrial matrix, is structurally complex and contains several subdomains: the inner boundary membrane, the cristae membrane, the cristae junctions and the cristae tips.

The outer membrane separates the mitochondrion from the rest of the cell and harbors the translocase of the outer membrane (TOM), which is the main entry gate of cytosol-encoded mitochondrial proteins, as well as the sorting and assembly machinery (SAM). Key proteins governing mitochondiral dynamics also reside in the outer mitochondrial membrane. The $\mathrm{OM}$ is populated by protein porins, commonly 

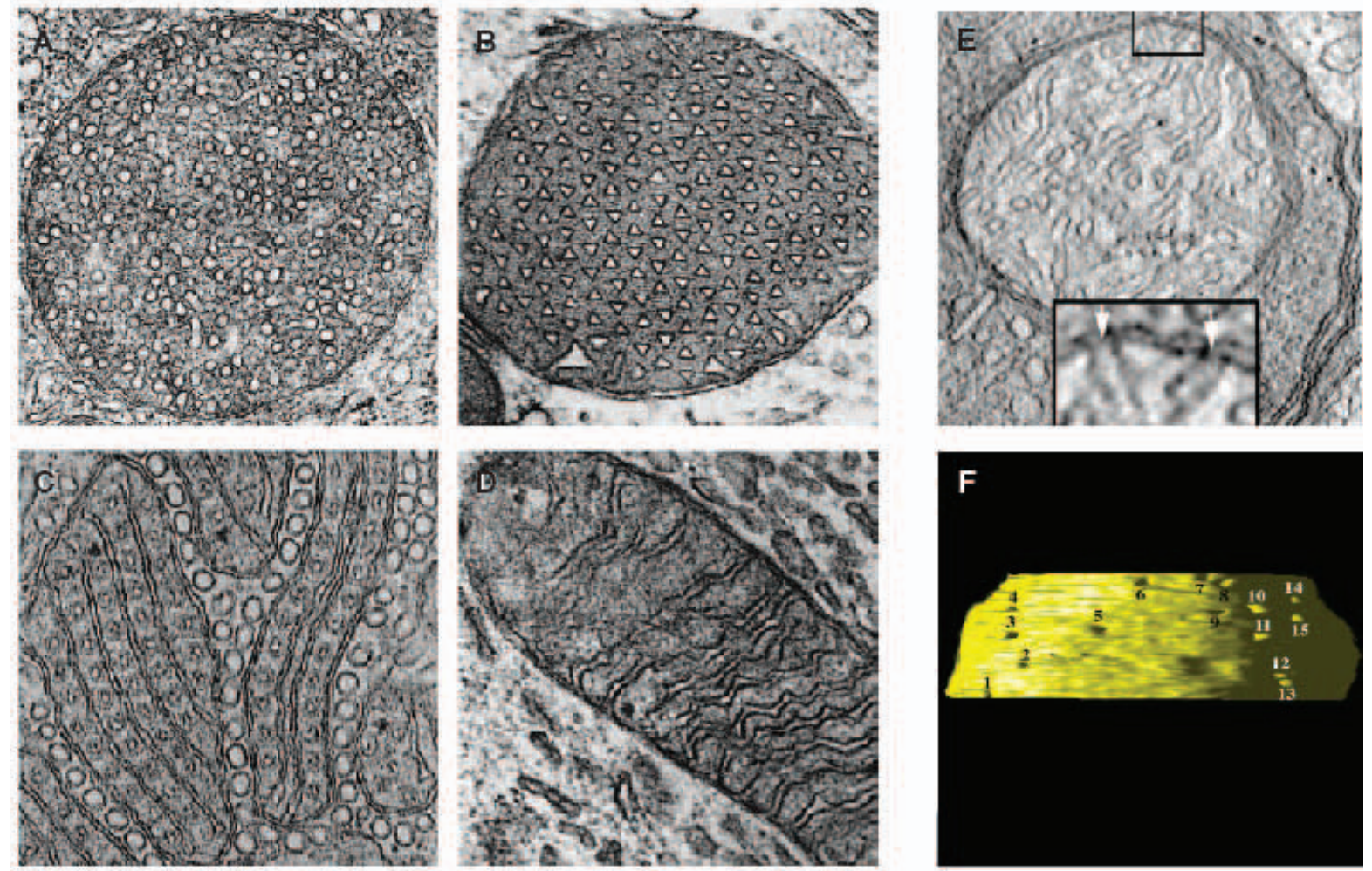

Figure 1.3: Diversity of the mitochondrial ultrastructure. Electron micrographs of mitochondria from different types of tissues/cells and physiological conditions display various cristae morphologies, whereas the cristae junctions remain uniform. A. Cristae of the adrenal cortex adopt vesicular shapes. B. Prismatic cristae (displayed as triangles because of the image cross-section) of astrocytes. C. Mitochondria from the pseudobranch of Cyprinoden variegatus, which was exposed to constant light for a period of time. In such conditions, mitochondria, in addition to normal foliate cristae, display secondary tubular cristae arranged in rows between the longitudinally oriented cristae. D. Ventricular cardiac muscle displays zigzag cristae morphology. E. Cristae junction (boxed) of mouse mitochondrion. The inset shows the two open (arrows) adjacent cristae junctions. F. The numbers indicate the cristae junctions which are uniform in diameter (A-D taken from (Fawcett, 1981; Zick et al., 2009b), $E$ and $F$ taken from (Perkins et al., 2012).

known as the voltage-dependent anion channel (VDAC), which allow the passage of ions and metabolites and thereby the $\mathrm{OM}$ cannot maintain a tight diffusion barrier (Figure 1.4) (van der Laan et al., 2012). 


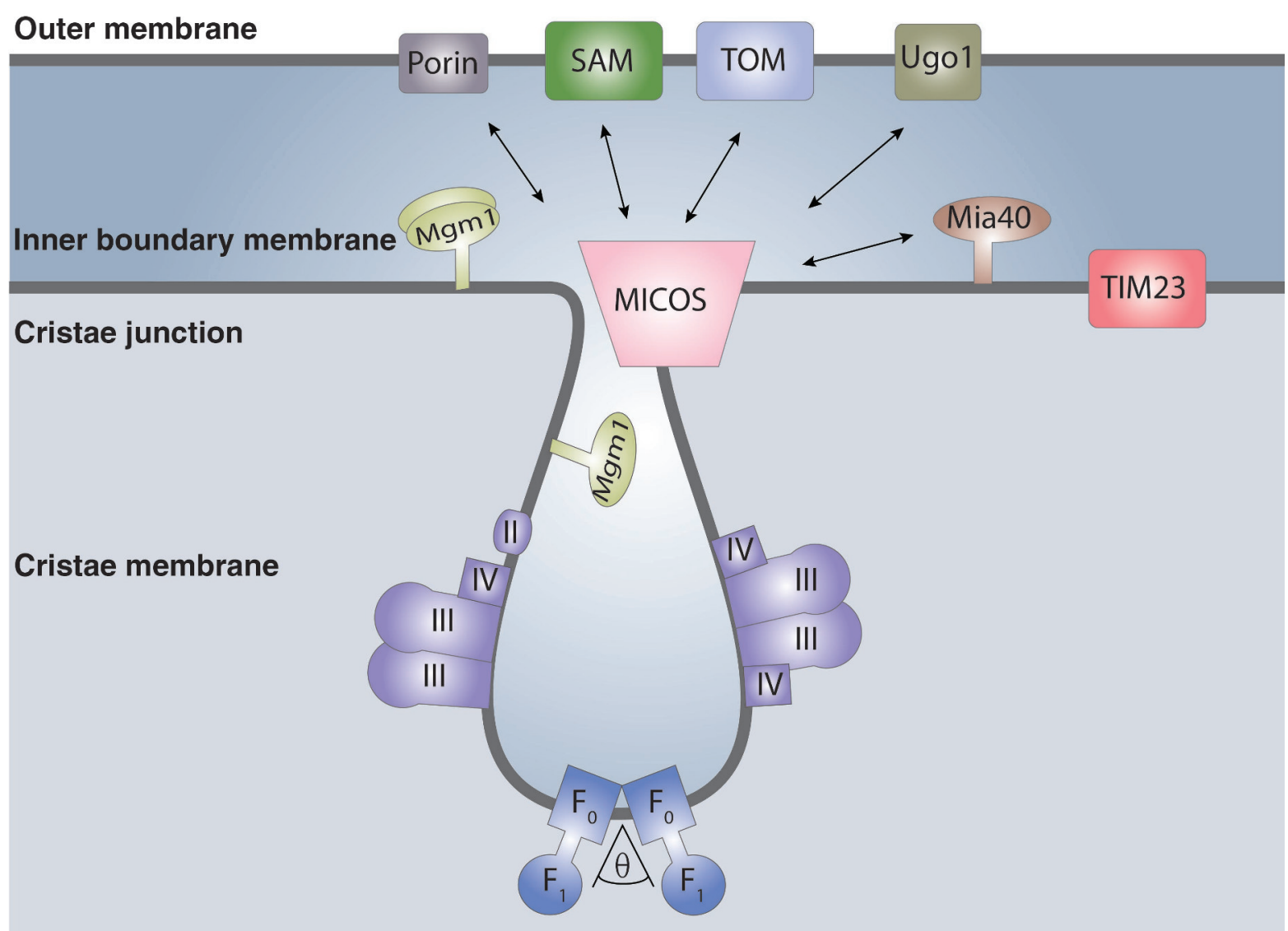

Figure 1.4: Mitochondrial inner membrane protein organization and distribution. Mitochondrial proteins are dynamically but unevenly enriched in different subdomains of the inner membrane. For example, protein transport machineries such as TIM23 and Mia40 and a soluble form of the fusion protien Mgm1 are present at the inner boundary membrane, whereas respiratory chain complexes II, III, IV are located at the cristae membranes. The $F_{1} F_{O}-A T P$ synthase is enriched at highly curved cristae tips and the long form of Mgm1 and the MICOS complex are enriched at cristae junctions. The MICOS complex interacts with various outer membrane protiens/protein complexes such as TOM, SAM, Porin, Mia40 and the outermembrane fusion protein Ugo1. Adapted from (van der Laan et al., 2012).

In contrast, the IM is tightly sealed in order to maintain the transmembrane proton gradient, which is ultimately consumed by the $F_{1} F_{O}$-ATP synthase for ATP generation. The inner membrane is populated by the translocases of the inner membrane (TIM23 and TIM22) and the mitochondrial intermembrane space assembly machinery (Mia40), as well as the components of fusion/fission machinery, the respiratory 
chain complexes and the $F_{1} F_{O}$-ATP synthase (Figure 1.4) (van der Laan et al., 2012).

The IM is highly folded and thereby has a much larger surface to volume ratio than the OM. This sophisticated convoluted structure gives rise to the subdivision of the inner membrane into different morphological regions, displaying different degress of membrane curvature: the inner boundary membrane (IBM), which is in close proximity to the $\mathrm{OM}$, and can be regarded as flat; the cristae membranes, which can adopt a variety of shapes from highly curved tubular structures to more flat lamellar, sheet-like structures; the cristae junctions (CJs), which are narrow, tubular apertures that connect the inner boundary and cristae membranes, and finally cristae tips or rims. Both CJs and cristae tips display high degrees of membrane curvature (Figure 1.2). Remarkably, although the cristae membranes are diverse in shape and size, depending on varying physiological conditions, the CJs are rather uniform with average diameters between 10 to $30 \mathrm{~nm}$ (Figure 1.3) (Frey and Mannella, 2000; Mannella, 2006). Cristae junctions are believed to be structural organizers within the IM and therefore have a significant impact on mitochondrial processes. Accumulating evidence suggests that inner mitochondrial membrane proteins are differentially distributed along the IM. For example, the protein translocase TIM23 has a preference for the IBM where it is in close contact with the OM translocase TOM. The components of the oxidative phosphorylation system on the other hand are enriched in the cristae membranes (Wurm and Jakobs, 2006; Vogel et al., 2006). Thus, the $\mathrm{IM}$ is divided into regions with specific protein content and specialized function (Mannella et al., 1994; Williams, 2000; Wurm and Jakobs, 2006; Vogel et al., 2006; Strauss et al., 2008; Stoldt et al., 2012; Jans et al., 2013). The importance of CJs in mitochondrial and cellular processes is further underlined by their role in establishing a diffusion barrier between the intracristae space and the intermembrane space (IMS). The rationale of such compartmentalization would be to limit or control the movement of metabolites such as protons or ADP and thus regulate the rate of ATP production (Mannella et al., 1994; Perkins et al., 1997; Frey and Mannella, 2000; Frey et al., 2002). Cristae junctions play a role in regulation of apoptosis by preventing the release of pro-apoptotic molecules such as cytochrome c from intracristae space to the cytosol (Scorrano et al., 2002; Pellegrini and Scorrano, 2007; Galluzzi et al., 2012). The second highly curved region are the cristae tips. Although their physiological role is poorly understood, it is suggested that such geometry of cristae tips facilitates the effective generation of electrochemical membrane potential and 
ultimate ATP production by oxidative phosphorylation (Strauss et al., 2008).

The peculiar morphology of the IM and the physiological importance of the integrity of the highly curved CJs and cristae tips suggest that tight protein scaffolds stabilize these structures. A number of proteins and protein complexes have been suggested to be involved in cristae biogenesis (Neupert, 2012). Below, only those that are directly implicated in cristae formation by shaping CJs and cristae tips will be discussed.

\subsubsection{Proteins underlying the inner mitochondrial membrane morphology}

\subsubsection{ATP synthase}

The F-ATP synthases are multisubunit protein complexes which are found in energytransducing membranes of eukaryotes, bacteria and archaea (Muench et al., 2011; Walker, 2013). They engage a transmembrane protonmotive force to produce cellular ATP from ADP and Pi. While their main role and overall mechanistic principles are well documented, other characteristics such as their role in mitochondrial IM formation and maintenance, and the underlying molecular mechanisms, are still poorly understood.

The $\mathrm{F}_{1} \mathrm{~F}_{\mathrm{O}}$-ATP synthase in yeast is composed of at least 17 subunits which compose the water-soluble, catalytic $F_{1}$ head and the membranous $F_{O}$ domain. The complex, analyzed from various species, has been shown to be arranged into dimer rows in cristae tips, revealing that these structures are ubiquitous features of mitochondria (Allen et al., 1989; Strauss et al., 2008; Davies et al., 2011).

Four subunits of the $F_{O}$ domain have been shown to be important for stepwise dimer formation: e,g-dimerization and k,i-supportive (Arnold et al., 1998; Wagner et al., 2009; Wagner et al., 2010). The subunits e and g possess one conserved putative transmembrane domain which contains conserved glycine-rich motifs $\mathrm{GxxxG}$ (two glycine residues are separated by any three amino acids). Such glycine-rich motifs are important for assembly of two transmembrane helices (Russ and Engelman, 2000).

The possible role of ATP synthase dimers in the generation of tubular cristae was first proposed by Allen (Allen, 1995). However, the direct link between ATP synthase dimerization and cristae biogenesis came from studies in yeast (Paumard et al., 
2002). These studies showed that depletion of oligomerization subunits e, g (su e, su g) or e/g inhibits dimer formation and results in an abnormal, onion-like inner mitochondrial membrane architecture (Figure 1.5) (Giraud et al., 2002; Paumard et al., 2002; Arselin et al., 2003; Zick et al., 2009a). Moreover, alterations in the GxxxG motifs lead to the loss of both subunits as well as to ATP dimer disruption, and ultimately abnormal inner mitochondrial membrane morphology in yeast. This confirms that dimers are a prerequisite for normal inner mitochondrial membrane morphology maintenance (Arselin et al., 2003; Bustos and Velours, 2005). The current generally accepted model for cristae tip formation by the ATP synthase suggests, that ATP synthase dimers generate a strong local positive curvature in the IM towards the matrix. The effective angle between two monomers of the dimer enables the dimer to form a rigid arc and thereby bend the membrane. Molecular dynamic simulation studies have also confirmed that ATP synthase dimers are sufficient to bend lipid bilayer membranes (Davies et al., 2012).

Recent structural studies of mammalian ATP synthase complexes solubilized with mild detergents or packed into $2 \mathrm{D}$ membrane crystals revealed that the ATP synthase monomers, and not the dimers might be the minimal bending module of lipid bilayers (Baker et al., 2012; Jiko et al., 2015). Whether the complexes in a native environment would exhibit the same characteristics is not known. Nonetheless, dimer rows seem to be the lowest-energy arrangement of the ATP synthase complex in the inner mitochondrial membrane (Baker et al., 2012).

Despite the apparent importance of ATP synthase dimers and dimer rows in cristae biogenesis, the loss of dimerization subunits, though it leads to reduced cell growth and membrane potential, is not lethal (Boyle et al., 1999; Bornhovd et al., 2006). ATP synthases from prokaryotes do not have the dimer-specific subunits and dimer rows. Consequently, prokaryotes do not contain cristae membranes. Thus, the oligomerization subunits appear to be dispensable for cell growth, but, are essential for crisate biogenesis (Kuhlbrandt, 2015).

Cardiolipin (CL) is a lipid dimer which consists of two phosphatidyl groups connected by a glycerol (Claypool and Koehler, 2012). This peculiar lipid is abundant in the inner mitochondrial membrane and has been implicated in membrane remodeling (Huang and Ramamurthi, 2010). This phospholipid has a tendency to cluster and its clusters have been shown to preferentially localize to the poles of bacterial cells (Mileykovskaya and Dowhan, 2000; Nishibori et al., 2005). Furthermore, ap- 

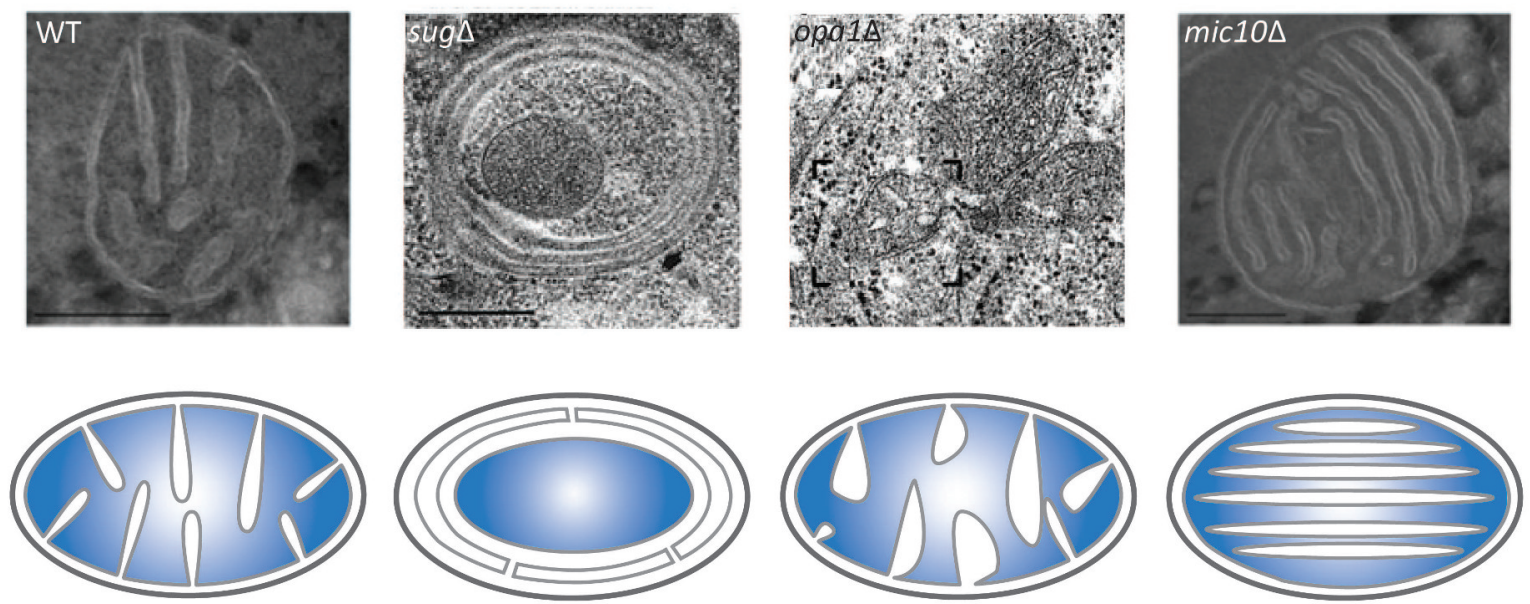

Figure 1.5: Role of various mitochondrial proteins in cristae morphology maintenance. The inner membrane of the wild-type mitochondrion displays charasteristic cristae structures, whereas the indicated protein-depleted mitochondria exhibit abnormal IM morpholoogies. In the absence of $F_{1} F_{O^{-}}$ ATP synthase dimerization subunit, the inner membrane exhibits the concentric onion-like shapes. Lack of OPA1 results in abberant cristae structures and depletion of MICOS core subunits leads to the drastic reduction of a number of CJs and thus to detachment of the cristae membrane from the inner boundary membrane. EM micrographs for WT and $\Delta$ micos are taken from (von der Malsburg et al., 2011), $\Delta$ sug from (Paumard et al., 2002) and $\Delta$ opa1 from (Olichon et al., 2003).

proximately $75 \%$ of cardiolipin has been shown to be present at the matrix side of the inner mitochondrial membrane (Krebs et al., 1979). Cardiolipin molecules have also been shown to be associated with ATP synthase molecules, which are enriched at cristae tips (Eble et al., 1990). This gives rise to the question whether the ATP synthase creates curvature in the inner membrane or just favors confinement in the curved regions. If cardiolipin molecules should form the cristae tips, because of their cone-like shape they would lead to strong negative curvature, quite opposite to what is observed. Interestingly, cardiolipin molecules play a chaperone-like function in the assembly of ATP synthase dimers and thereby indirectly affect the cristae morphology (Acehan et al., 2011). Thus, the interplay of cardiolipin and the ATP synthase is important in the formation and maintenance of the highly curved cristae tips. 


\subsubsection{Mgm1/OPA1}

Another protein which is likely to be responsible for maintaining the inner mitochondrial membrane architecture is Mgm1 (mitochondrial genome maintenance) in yeast and its mammalian homolog OPA1 (optic atrophy 1), one of the key proteins of the mitochondrial fusion machinery (Wong et al., 2003; Cipolat et al., 2004; Meeusen et al., 2006; Westermann, 2010). Mgm1 exists in two forms: a long, N-terminally inner mitochondrial membrane-anchored (I-Mgm1) and a short, soluble (s-Mgm1) form, yet both forms are generated from the same precursor protein by different proteolytic cleavage (Esser et al., 2002; Herlan et al., 2003). In contrast to Mgm1, OPA1 has at least eight different splice variants, which, after proteolytical processing are also divided into short and long forms (Delettre et al., 2001; Cipolat et al., 2006). Mgm1/OPA1 are large GTPases of the dynamin superfamily and their coordinated action leads to mitochondrial fusion. Mutations in this protein cause dominant optic atrophy in mammals, underlying the physiological importance of mitochondrial dynamics (Delettre et al., 2001).

Depletion of Mgm1/OPA1 leads to abnormal inner mitochondrial membrane morphology, with unfolded/aberrant or reduced number of cristae (Figure 1.5) (Olichon et al., 2002; Sesaki et al., 2003; Amutha et al., 2004). In addition to and independent from its role in fusion, OPA1 is known for its role in intrinsic apoptosis. OPA1 protects cells from apoptosis upon apoptotic stimuli by sequestering the release of pro-apoptotic soluble molecules, such as cytochrome c, from the intracristae space to cytosol, by controlling cristae remodeling (Scorrano et al., 2002; Olichon et al., 2002). In fact, downregulation of OPA1, leading to abnormal cristae morphologies with widened CJs upon apoptotic stimuli, results in accelerated cytochrome c release. Overexpression of OPA1 is suggested to protect cells from death by tightening CJs. Recent studies demonstrated that controlled overexpression of OPA1 has a protective effect in a number of mouse disease models related to apoptotic tissue damage (Civiletto et al., 2015; Varanita et al., 2015).

How does Mgm1/OPA1 regulate cristae remodeling? Similar to other dynaminrelated proteins, Mgm1/OPA1 also oligomerizes. Mgm1/OPA1 forms homo- as well as hetero-oligomers in its short and long forms. OPA1 mainly exists in trimetic hetero-oligomeric complexes, comprising two long and one short OPA1 (Frezza et al., 2006). The levels of short OPA1 in the oligomers are crucial for oligomer 
formation (Cipolat et al., 2006). In addition, Mgm1/OPA1 oligomerization is necessary to maintain physiological cristae morphology (Danino and Hinshaw, 2001; Frezza et al., 2006; Zick et al., 2009a). Thus, the stoichiometry of two different forms of Mgm1/OPA1 determines protein oligomerization that subsequently defines physiological cristae morphology.

It has been reported that soluble OPA1 can promote tubulation of liposomes (Ban) et al., 2010). Soluble Mgm1 assembles on membranes in crystalline arrays and, like its mammalian homolog, induces liposome deformation (DeVay et al., 2009; Rujiviphat et al., 2015). Soluble Mgm1/OPA1 specifically binds to negatively charged non-bilayer forming lipids such as cardiolipin, phosphatidylserine (PS) and phosphatidic acid (PA). s-Mgm1 clusters cardiolipin and locally affects model membrane stability (Rujiviphat et al., 2015), whereas similar effects for I-Mgm1 have not been reported. Although s-Mgm1/s-OPA1 possesses an intrinsic affinity to membranes as well as the ability to affect their morphology, it is not clear how it would generate and maintain cristae junctions in mitochondria. Soluble Mgm1/OPA1 probably affects membrane stability in a way similar to dynamin I, whose effects on membranes are transient. As cristae junctions are dynamic but permanent structures, their generation or stabilization would require a membrane-anchored protein, in a similar way to, for example, the dimerization subunits of the $F_{1} F_{O}$-ATP synthase for the cristae tip (Kuhlbrandt, 2015) and reticulons and DP1/Yop1p for the endoplasmic reticulum (ER) formation and maintenance (Voeltz et al., 2006; Hu et al., 2008). Therefore, Mgm1/OPA1 hetero-oligomers are probably required to maintain cristae junctions. It could be that the long form of Mgm1/OPA1 anchored in the cristae membrane provides scaffolds at cristae structures, whereas short mechanoenzyme Mgm1/OPA1 could promote nucleotide dependent non-bilayer lipid clustering and local curvature production (Rujiviphat et al., 2015). Thus, a possible stabilization of tubular CJ might be achieved by cross-linking different sides of cristae membrane through hetero-oligomerization.

Mgm1 has been shown to have a chaperone-like function for the ATP synthase dimerization subunits (Amutha et al., 2004; Mannella, 2008). Yeast cells that lack Mgm1 exhibited reduced levels of ATP synthase dimerization subunits, whose assembly into oligomers is important for cristae membrane morphology maintenance as described in section 1.4.1.1. Thus, the abnormal cristae membrane phenotypes 
are likely a result of the alterations in the levels of ATP synthase dimerization subunits (Amutha et al., 2004). On the other hand, depletion of prohibitins, which are involved in proteolytic processing of OPA1, causes defective cristae morphogenesis (Merkwirth et al., 2008; Merkwirth and Langer, 2009; Osman et al., 2009). This points to a more direct role of OPA1 in cristae maintenance.

\subsubsection{MICOS complex}

The recent discovery of the evolutionarily conserved multi-subunit protein complex termed mitochondrial contact site and cristae organizing system (MICOS) brought a new perspective into the understanding of cristae junction formation. Remarkably, the complex was identified by four independent studies and hence, originally received various names: mitochondrial inner membrane organizing system (MINOS), mitochondrial organizing structure (MitOS) and MICOS (Hoppins et al., 2011; Harner et al., 2011; von der Malsburg et al., 2011; Alkhaja et al., 2012). Recently, a unifying nomenclature has been proposed and the complex is termed MICOS, with its subunits Mic10 to Mic60, where the numbers represent the approximate molecular size of the proteins (Pfanner et al., 2014). In yeast the MICOS complex consists of at

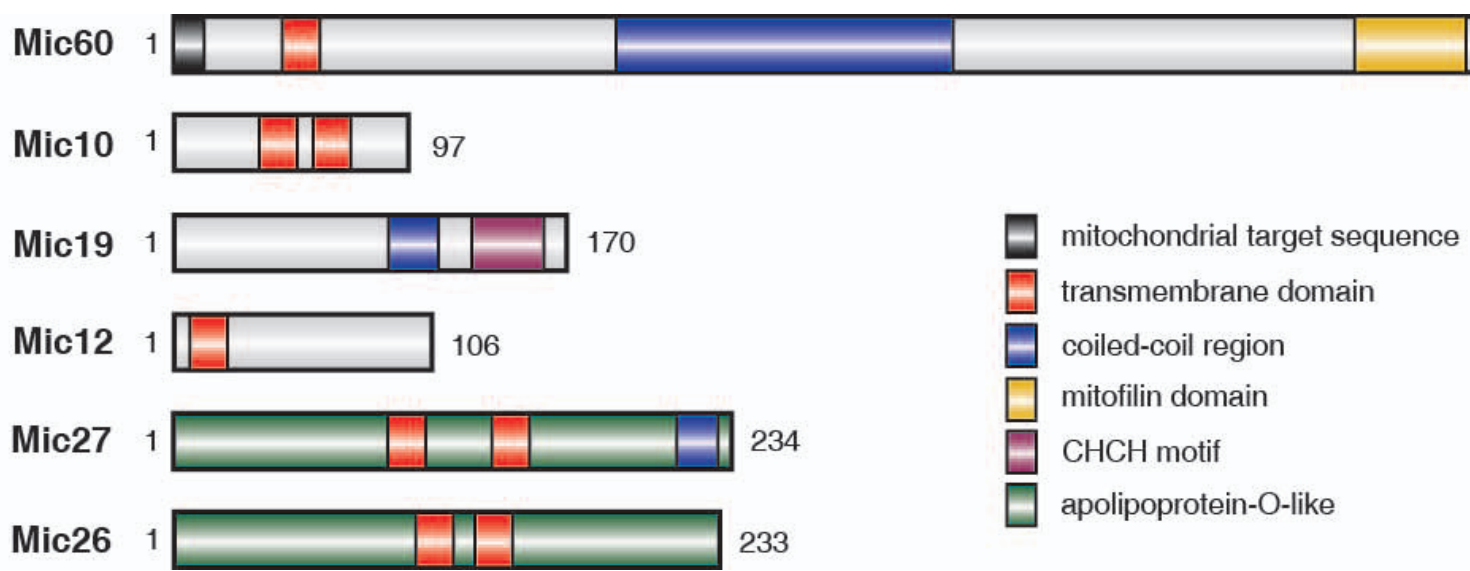

Figure 1.6: Composition of MICOS complex. The MICOS complex consists of six membrane proteins. The different protein domains are indicated. These subunits represent the proteins from $S$. cerevisiae.

least 6 different subunits, of which the majority are integral membrane proteins with one or two transmembrane domains (Figure 1.6) (Hoppins et al., 2011; Harner et al., 
2011; von der Malsburg et al., 2011; Alkhaja et al., 2012; van der Laan et al., 2012). Two of the subunits, Mic60 and Mic10, form the core of the complex. Depletion of MICOS subunits leads to dissociation of the MICOS complex and to abnormal inner mitochondrial membrane morphology, with stacked cristae and a massive loss of cristae junctions (Figure 1.5). This suggests a role of the complex in mitochondrial IM architecture (Hoppins et al., 2011; Harner et al., 2011; von der Malsburg et al., 2011; Alkhaja et al., 2012; Zerbes et al., 2012b).

Although the existence of MICOS as a holocomplex has been suggested to play a pivotal part in its role in mitochondria, recent studies have challenged this view. These studies reported the existence of two independent MICOS subcomplexes and their possible different roles in mitochondrial processes (Harner et al., 2014; Friedman et al., 2015; Bohnert et al., 2015b; Guarani et al., 2015; Zerbes et al., 2016).

Below I will summarize the accumulated knowledge about the yeast MICOS complex and its single subunits, and parallels between the yeast subunits and their mammalian homologs/orthologs will be made.

Mic60: Human homolog MIC60 was formerly termed mitofilin because of its mitochondrial localization and filamentous appearance in fibroblasts (Odgren et al., 1996; Gieffers et al., 1997). Before its identification as a part of the MICOS complex, MIC60/mitofilin was already implicated in mitochondrial IM morphology maintenance by mainly homotypic interactions (John et al., 2005; Rabl et al., 2009). Mic60 possesses an $\mathrm{N}$-terminal mitochondrial targeting signal followed by a single transmembrane domain by which it is anchored in the cristae membrane. A large portion of the C-terminus is exposed towards the IMS (Rabl et al., 2009). Depletion of Mic60 in different species leads to abnormal inner mitochondrial membrane architecture with a massive loss of cristae junctions and aberrant cristae structures (John et al., 2005; Mun et al., 2010; von der Malsburg et al., 2011). These observations indicate a conserved function of Mic60 in inner mitochondrial membrane morphology maintenance by stabilizing cristae junctions. Furthemore, overexpression of Mic60 in yeast leads to increased branching of cristae membranes (Rabl et al., 2009; Bohnert et al., 2015b). Although MICOS subunits are highly conserved in eukaryotes from yeast to mammals, Mic60 is the only protein, that has a homolog in $\alpha$-proteobacteria, the prokaryotic ancestor of mitochondria (Munoz-Gomez et al., 2015).

Along with other MICOS subunits, Mic60, through its large IMS domain, forms 
contact sites with various outer membrane proteins and protein complexes such as TOM, SAM, porin and Ugo1 (Xie et al., 2007; Hoppins et al., 2011; Harner et al., 2011; von der Malsburg et al., 2011; Ott et al., 2012; Körner et al., 2012; Zerbes et al., 2012a; Bohnert et al., 2012; Ding et al., 2015). The interaction of Mic60 with TOM promotes the import of intermembrane space and $\beta$-barrel precursor proteins into mitochondria. This function of Mic60 is independent from its integration into the MICOS holocomplex (von der Malsburg et al., 2011; Bohnert et al., 2012; Varabyova et al., 2013). The connection between Mic60 and Ugo1 might suggest the involvement of Mic60 in mitochondrial fission (Harner et al., 2011). Thus, Mic60 appears to adopt dual MICOS-dependent (cristae maintenance) and MICOS-independent (protein biogenesis) functions (Horvath et al., 2015). As already mentioned, recent studies in yeast have succeeded in identifying two independent MICOS subcomplexes, Mic60/Mic19 and Mic27/Mic10/Mic12. These complexes have non-redundant functions within mitochondria (Friedman et al., 2015). Based on the above information, it is possible that the Mic60 MICOS-independent function is found within the Mic60/Mic19 subcomplex.

Depletion of Ugo1 and Sam50 results in altered IM morphology, suggesting the importance of Mic60/MICOS interaction with the outer membrane proteins for inner mitochondrial membrane morphology maintenance (Harner et al., 2011; Ott et al., 2012). The OM proteins might play a role in proper positioning of Mic60 and/or the MICOS complex in the IM. Hence, Mic60 might influence the mitochondrial cristae organization as a part of the MICOS complex and by interacting with OM proteins (Horvath et al., 2015; Friedman et al., 2015).

Mic10: Mic10 is a small integral membrane protein, which is targeted to mitochondria by conserved positive charges between two predicited transmembrane domains (Bohnert et al., 2015b). It is a core component of the MICOS complex along with Mic60. Deletion of Mic10 severely affects the stability of MICOS and leads to drastic alterations of IM morphology with a reduced number of CJs (Hoppins et al., 2011; Harner et al., 2011; von der Malsburg et al., 2011; Alkhaja et al., 2012; Bohnert et al., 2015b). The absence of Mic10 results in impaired cell growth, indicating the importance of the protein in cell viability (Alkhaja et al., 2012). Overexpression of Mic10 in budding yeast triggers morphological alterations of the inner membrane with elongated cristae membranes, suggesting its ability to affect membrane mor- 
phology (Bohnert et al., 2015b).

Although the importance of Mic10 in the maintenance of MICOS complex integrity as well as IM morphology is apparent, little is known about its direct role in cristae formation.

Mic19/MIC19/CHCHD3: Mic19 is the only MICOS subunit that lacks the apparent transmembrane segments. Instead, it contains a predicted $\mathrm{N}$-terminal myristoylation site followed by a coiled-coil region and the C-terminal $\mathrm{CHCH}$ (Coiled-coil helix coiled-coil helix) domain (Darshi et al., 2011; von der Malsburg et al., 2011). Human MIC19 has an additional $\mathrm{N}$-terminal phosphorylation site with so far unknown function (Schauble et al., 2007). In contrast to its human ortholog, which contains classical MIA substrate twin Cys- $\mathrm{X}_{9}-\mathrm{Cys}_{\mathrm{s}}$ motif in its $\mathrm{CHCH}$ domain, the yeast Mic19 possesses an atypical single Cys- $\mathrm{X}_{10}$-Cys motif. However, like MIC19, Mic19 also engages in the mitochondrial IMS import and assembly machinery (MIA) pathway (Darshi et al., 2012; Sakowska et al., 2015). Interestingly, MIC19, unlike other $\mathrm{CHCH}$ domain proteins, requires myristoylation as well as the $\mathrm{CHCH}$ domain for its mitochondrial localization (Darshi et al., 2012).

In both mammals and yeast, the downregulation of Mic19 results in a partial dissociation of the MICOS complex and altered inner mitochondrial membrane morphology with stacked cristae and a partial loss of cristae junctions (Darshi et al., 2011; Hoppins et al., 2011; Harner et al., 2011; von der Malsburg et al., 2011; Sakowska et al., 2015).

Recently, it was shown that Mic19 acts as a connector between the two MICOS subcomplexes and maintains the integrity of the MICOS complex (Friedman et al., 2015). Follow-up studies confirmed the latter and provided more insight into the regulation. Sakowska et al showed that Mic19 occurs in two different intramolecular and intermolecular oxidized forms in mitochondria (Sakowska et al., 2015). The intramolecular disulfide bonds formed by cystein oxidation are required for functionally mature proteins (Webb et al., 2006; Banci et al., 2008). In fact, the intramolecular oxidized state of Mic19 regulates the integrity of the MICOS complex and thus, the maintenance of normal inner mitochondrial membrane morphology. This was addressed by analyzing the ultrastructure and MICOS complex composition of mitochondria contaning reduced Mic19 (Mic19C146S). Such mitochondria

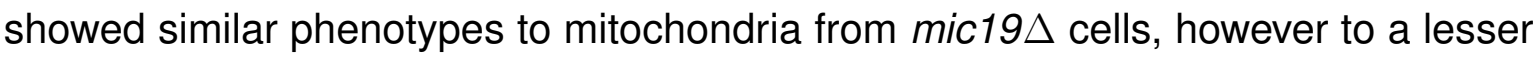


extent. Moreover, the integrity of the MICOS complex was compromised, with Mic10, Mic12 and Mic26 occurring as a MICOS-independent Mic10/Mic12/Mic26 subcomplex (Sakowska et al., 2015). It has to be noted that the original subcomplex identified by Friedman et al contained Mic27 instead of Mic26, however, these proteins are homologs and in mammals their roles are interchangeable (see below). The function of the intermolecularly oxidized form has not yet been experimentally addressed, however, it was proposed that it might regulate the number of intramolecularly oxidized Mic19 and thus determine the copy number of cristae junctions (Sakowska et al., 2015).

Mammalian MIC19 plays a role in inner membrane morphology maintenance by controlling the homeostasis of MIC60, one of the MICOS core subunits (Li et al., 2015). MIC60 is a substrate of the mitochondrial protein quality control i-AAA protease Yme1L, which is located in the mitochondrial inner membrane (Baker et al., 2011; Anand et al., 2014). Cells that lack MIC19 showed reduced levels of Mic60. The loss of Yme1L in the same cells rescued the levels of the MIC60. MIC19 and Yme1L have the same binding site for MIC60. Hence, it is conceivable that MIC19 maintains cellular MIC60 levels by preventing MIC60 from Yme1L recognition and degradation (Li et al., 2015). Interestingly, cells lacking Mic19 in yeast also exhibit reduced levels of Mic60, however a similar regulatory role of Mic19 has not been described yet (Harner et al., 2011).

Mic12/QIL1: Mic12 is an integral membrane protein with a predicted N-terminal transmembrane domain and its soluble portion is exposed to the IMS (Hoppins et al., 2011; Harner et al., 2011; von der Malsburg et al., 2011). Absence of Mic12 leads to a partial disassembly of MICOS and a mild IM morphology phenotype. In general this protein is the least studied one of the MICOS complex.

Mic12 was thought to be the only MICOS protein with no non-fungal ortholog (Zerbes et al., 2012b; Huynen et al., 2016). Huynen et al showed that QIL1, a recently identified and characterized mammalian protein is an ortholog of Mic12, however their sequence similarity is very low beyond the predicted transmembrane domain (Huynen et al., 2016). QIL1 is an apparent component of the MICOS complex. Depletion of the protein leads to abnormal inner mitochondrial membrane structures with increased cristae swirls. Moreover, QIL1 was shown to be important for MIC10 integration into the MICOS complex (Guarani et al., 2015). 
Mic26 and Mic27: Like their mammalian orthologs formerly termed APOO (apolipoprotein O) and APOOL (apolipoprotein O-like), yeast Mic26 and Mic27 are also defined as apolipoproteins (Lamant et al., 2006; Hoppins et al., 2011; Weber et al., 2013). Although classical apolipoproteins are located extracellularly, MICOS apolipoproteins are found in mitochondria. APOO was recently identified as a genuine constituent of mammalian MICOS and renamed to MIC26 (Koob et al., 2015). However, it is currently debated whether MIC26 is orthologous to Mic26 or to Mic27 and another recent report suggested renaming APOO to MIC23 (Ott et al., 2015). Both proteins contain two putative transmembrane segments and a predicted coiled-coil domain exposed to the IMS side of the IM (Lamant et al., 2006; Hoppins et al., 2011; Weber et al., 2013). How they are targeted to mitochondria is not known. Mammalian MIC27 was reported to have an N-terminal mitochondrial targeting signal, albeit a mitochondrial processing site has not yet been identified (Weber et al., 2013). Interestingly, MIC23/MIC26 was found in two different forms: a non-glycosylated $22 \mathrm{KDa}$ form (mitochondrial) and a glycosylated $55 \mathrm{kDa}$ form (non-mitochondrial), whereas for the yeast orthologs similar characteristics have not been observed (Koob et al., 2015). Yeast and mammalian Mic26 and Mic27 are inner membrane proteins. However, MOMA-1, their homolog in $C$. elegans, was found in the OM as well as in the IM (Huh et al., 2003; von der Malsburg et al., 2011; Head et al., 2011; Weber et al., 2013; Koob et al., 2015).

Downregulation of mammalian MIC23 leads to an abnormal inner membrane architecture with reduced number of CJs and broader cristae, whereas absence of MIC27 results in fragmented mitochondria and concentric cristae structures (Weber et al., 2013; Koob et al., 2015). In yeast only the deletion of Mic27 leads to abnormal IM architecture: the lack of Mic26 has no apparent effect on IM morphology (Hoppins et al., 2011; Harner et al., 2011; von der Malsburg et al., 2011). This suggests that despite their homology the roles of yeast Mic26 and Mic27 in CJ formation are distinct.

Mammalian MIC23 and MIC27 regulate their levels in an antagonistic manner, which has not been shown for the yeast orthologs (Koob et al., 2015). This suggests that the role of MIC23 and MIC27 in IM morphology maintenance might be redundant. Nonetheless, IM morphology is impaired when the balanced overexpression of both MIC23 and MIC27 is altered, it is unlikely that they are functionally fully redundant (Koob et al., 2015). Recombinantly expressed MIC27 specifically binds to 
cardiolipin. On the other hand, downregulation of MIC23 affects the steady state levels of taffazin, an enzyme that is required for CL acyl chain remodeling (Schlame, 2013). In yeast, CL metabolism is shown to be MICOS-dependent (Harner et al., 2014). Conversely, the assembly of the MICOS subcomplex Mic27/Mic10/Mic12 is cardiolipin-dependent (Friedman et al., 2015). Thus, the functions of Mic27/MIC27 and Mic26/MIC23 are not redundant but interdependent and their role in inner mitochondrial membrane maintenance is probably connected to cardiolipin.

In addition to their other important roles, apolipoproteins are mainly involved in lipid transport (Bolanos-Garcia and Miguel, 2003). Therefore, the aforementioned ability of MIC27 to bind cardiolipin and the role of MIC23 in controlling tafazzin levels lead to the assumption that these proteins might be involved in $\mathrm{CJ}$ formation by transporting and/or modulating the levels of CL. Koob et al suggested that MIC27 plays a role in transporting CL between the IM and the OM and from the IBM to the cristae membrane (Koob and Reichert, 2014). At cristae membranes CL is known to enhance the stability of respiratory chain supercomplexes and of the $F_{1} F_{O}$-ATP synthase, which is crucial for IM morphology maintenance (see section 1.4.1.1 (Schagger et al., 2004; Acehan et al., 2011). Another plausible suggestion is that MIC27 regulates $C L$ remodeling in highly curved areas such as CJs and cristae tips. Cardiolipin is a non-bilayer lipid, that clusters and in the presence of divalent cations forms hexagonal phases (Ortiz et al., 1999). Based on these properties and the observation that CL is enriched at cristae membranes at the matrix side, it is likely that this lipid is involved in forming highly curved areas of IM at CJs (Ortiz et al., 1999; Krebs et al., 1979; Koob and Reichert, 2014).

In conclusion, although a direct role of MICOS apolipoproteins in IM morphology generation and stabilization is not fully understood, the available data strongly suggests a CL recruitment by these proteins in the process of CJ formation. 



\section{Aim of this Work}

Proper organization of the inner mitochondrial membrane is essential for function of mitochondria. The morphology of the inner membrane is highly accepted to be regulated by the recently identified multisubunit protein complex MICOS which is enriched at cristae junctions. Absence of MICOS subunits, in particular Mic60 and Mic10, leads to severe alterations of the inner mitochondrial membrane morphology, resulting in abnormal cristae structures and a drastic reduction in the number of cristae junctions.

A direct role and the underlying molecular mechanism of MICOS subunits in the generation and stabilization of cristae junctions remains elusive. The functional characteristics of the MICOS core subunit, Mic60, in mitochondria is relatively welldocumented. In contrast, very little is known about the role of another MICOS core subunit, Mic10, in the generation of cristae junctions.

Hence, the aim of this study is to unravel the role of Mic10 in mitochondrial cristae formation and maintenance. For this purpose, I first investigate whether Mic10 is able to induce morphological changes in membranes. By integrating recombinant Mic10 into different types of model membranes I visualize the Mic10-induced structural changes of the membranes using electron and confocal fluorescence microscopy. In order to understand the mechanism of cristae junction formation and stabilization by Mic10 in vivo, I unravel the molecular mechanism by which Mic10 induces membrane curvature. This is addressed by determining the Mic10 topology in the inner mitochondrial membrane by using site-directed cystein labeling and subsequent size-shift detection as well as by addressing the ability of Mic10 to form oligomers. Finally, to show that Mic10-induced membrane bending is necessary for cristae junction formation in vivo, Mic10 mutant variants are designed that do not oligomerize and are expressed in yeast cells lacking wild type Mic10. The ultrastructure of such mitochondria is analyzed by electron microscopy to observe if they rescue the knock out phenotype of mic10. 



\section{Materials and Methods}

\subsection{Materials}

\subsubsection{Chemicals and lipids}

Standard chemicals and consumables were purchased from AppliChem (Darmstadt, Germany), Roth (Karlsruhe, Germany), Sarstedt (Nümbrecht, Germany), Sigma Aldrich (Taufkirchen, Germany). The specific reagents and commercial kits used in this study are listed in Table 3.1 and Table 3.2, respectively. Oligonucleotides were synthesized by Metabion (Martinsried, Germany). The lipids used in this study are listed in Table 3.3 .

Table 3.1: Reagents and their suppliers used in this study

\begin{tabular}{ll}
\hline Reagent & Supplier \\
\hline Acyl-N-methylglucamine 9 (Mega 9) & Glycon \\
Ampicillin & Roth \\
Bio-Beads SM-2 Adsorbent & Roth \\
Bio-Rad protein assay & Roth \\
Chloroform & Merck \\
Complete amino acid supplement & MP Biomedicals \\
mixture without histidine & \\
n-Decyl- $\beta$-D-maltopyranoside (DM) & Glycon \\
Digitonin & Calbiochem/Merck \\
Dimethyl sulfoxide (DMSO) & Thermo Scientific \\
Dithiothreitol (DTT) & Roth \\
n-Dodecyl $\beta$-D-maltoside (DDM) & Glycon \\
Enhanced chemiluminescence (ECL) & Thermo Scientific \\
Western Blotting Substrate & \\
\hline
\end{tabular}




\begin{tabular}{|c|c|}
\hline Reagent & Supplier \\
\hline Ethidiumbromide $0.07 \%$ & AppliChem \\
\hline Fluorescent probes & Life Technologies \\
\hline Goat $\alpha$ Mouse Horseradish peroxidase (HRP) & Dianova \\
\hline $\begin{array}{l}\text { Goat } \alpha \text { Mouse Immunoglobulin G (lgG) } \\
\text { (whole molecule)-Gold antibody }\end{array}$ & Sigma Aldrich \\
\hline Goat $\alpha$ Rabbit HRP & Dianova \\
\hline Guanidine hydrochloride & Roth \\
\hline Hering Sperm DNA & Promega \\
\hline Histodenz & Sigma Aldrich \\
\hline Imidazole & Roth \\
\hline Isopropyl $\beta$-D-1-thiogalactopyranoside (IPTG) & Roth \\
\hline Kanamycin & Roth \\
\hline L-Glutathione reduced & Sigma Aldrich \\
\hline $\begin{array}{l}\text { Luminata Western Chemiluminescent } \\
\text { HRP Substrates }\end{array}$ & Millipore Corporation \\
\hline Lysozyme & Sigma Aldrich \\
\hline Magnesiumchloride $\left(\mathrm{MgCl}_{2}\right)$ & Roth \\
\hline $\begin{array}{l}\text { Minimum essential medium } \\
\text { (MEM) Vitamins solution }\end{array}$ & Life Technologies \\
\hline Mouse $\alpha$-His & Sigma Aldrich \\
\hline Phenylmethanesulfonyl fluoride (PMSF) & Sigma Aldrich \\
\hline $\begin{array}{l}\text { Poly(ethylene glycol) methyl } \\
\text { ether maleimide } 2 \mathrm{KDa}\end{array}$ & Sigma Aldrich \\
\hline Polyethylene glycol (PEG)-400 & Merck \\
\hline Polyvinylidene fluoride (PVDF) & Maine Manufacturing \\
\hline transfer membrane & LLC \\
\hline Proteinase inhibitor cocktail (EDTA-free) & Roche Applied Science \\
\hline Proteinase K (PK) & Roche \\
\hline Triton X-100 & Sigma Aldrich \\
\hline Tween-20 & Roth \\
\hline Rubidiumchloride (RbCl) & Sigma Aldrich \\
\hline
\end{tabular}




\begin{tabular}{ll}
...continued & \\
\hline Reagent & Supplier \\
\hline Urea & Roth \\
X-Ray films & GE Healthcare \\
Zymolyase 20T & Seikagaku Biobusiness \\
& Corporation
\end{tabular}

Table 3.2: Commercial kits and their suppliers

\begin{tabular}{ll}
\hline Commercial kit & Supplier \\
\hline Deoxynucleotide triphosphate mix & 5 PRIME \\
Deoxyribonuclease I (Dnase I) & Sigma Aldrich \\
Fluorescence labeling kit & Invitrogen \\
GeneRuler DNA ladder $1 \mathrm{~kb}$ & Fermentas/Thermo Scientific \\
HMW calibration kit & GE Healthcare \\
IgG protein standard & Bio-Rad \\
Mark12 Unstained Standard & Novex \\
NativeMarker unstained protein standard & Novex \\
NativePAGE Bis-Tris gel & Novex \\
Ni-NTA-agarose & 5 PRIME \\
NuPAGE Bis-Tris precast gel & Novex \\
PageRuler Prestained Protein Ladder & Thermo Scientific \\
Phusion DNA polymerase & Thermo Scientific \\
Restriction endonucleases & Thermo Scientific \\
T4 DNA ligase & Thermo Scientific \\
Wizard SV Gel and PCR Clean-UP & Promega \\
Wizard SV Mini-Prep & Promega \\
& \\
\hline
\end{tabular}


Table 3.3: Lipids and their manufacturers

\begin{tabular}{ll}
\hline Lipid & Manufacturer \\
\hline Cardiolipin & Avanti Polar Lipids \\
1,2-dimyristoyl-sn-glycero-3-phosphocholine & Sigma Aldrich \\
(DMPC) & \\
$\begin{array}{l}\text { 1,2-dioleoyl-sn-glycero-3- } \\
\text { phosphoethanolamine (DOPE) }\end{array}$ & Avanti Polar Lipids \\
L- $\alpha$-phosphatidylcholine (L- $\alpha$-PC) & Avanti Polar Lipids \\
L- $\alpha$-phosphatidylethanolamine (L- $\alpha$-PE) & Avanti Polar Lipids \\
L- $\alpha$-Phosphatidylethanolamine-N-(lissamine & Avanti Polar Lipids \\
rhodamine B sulfonyl) & \\
(Ammonium Salt) (rhodamine PE) & \\
L- $\alpha$-phosphatidylinositol (L- $\alpha$-PI) & Avanti Polar Lipids \\
L- $\alpha$-phosphatidylserine (L- $\alpha$-PS) & Avanti Polar Lipids \\
& \\
\hline
\end{tabular}

\subsubsection{Antibodies}

Rabbit polyclonal antisera produced at Gramsch Laboratories (Schwabhausen, Germany) were used as primary antibodies for immunodetection of Saccharomyces cerevisiae proteins. All used primary antibodies are listed in Table 3.4 .

Table 3.4: Antibodies used in this study

\begin{tabular}{lll}
\hline Antigen & Antibody type & Source \\
\hline$\alpha$-Aco1 & Rabit polyclonal & Gramsch Laboratories \\
$\alpha$-Mcr1 & Rabit polyclonal & Gramsch Laboratories \\
$\alpha$-Mic10 & Rabit polyclonal & Gramsch Laboratories \\
$\alpha-\operatorname{Tim} 21$ & Rabit polyclonal & Gramsch Laboratories \\
$\alpha-\operatorname{Tim} 23$ & Rabit polyclonal & Gramsch Laboratories \\
$\alpha-\operatorname{Tim} 44$ & Rabit polyclonal & Gramsch Laboratories \\
$\alpha$-Tom70 & Rabit polyclonal & Gramsch Laboratories
\end{tabular}




\subsubsection{Microorganisms}

E.coli strains for cloning, site-directed mutagenesis and protein expression are listed in Table 3.5. Saccharomyces cerevisiae BY4741 and its derivative mic10 $\Delta$ were used as major yeast strains. Further derivatives are listed in Table 3.6.

\section{Table 3.5: E.coli strains}

\begin{tabular}{lll}
\hline E.coli strain & Genotype & Source \\
\hline BL21 (DE3) & recA1 endA1 gyrA96 thi-1 hsdR17 supE44 relA1 & Stratagene \\
& lac $\left[\mathrm{F}^{\prime}\right.$ proAB lacl $\left.{ }^{9} \mathrm{Z} \Delta \mathrm{M} 15 \mathrm{Tn10}\left(\mathrm{Tet}^{r}\right)\right]$ & \\
$\mathrm{XL1}$ Blue & $\mathrm{F}^{-} \mathrm{dcm}$ ompT hsdS $\left(\mathrm{r}_{\mathrm{B}}{ }^{-} \mathrm{m}_{\mathrm{B}}{ }^{-}\right)$gal & Stratagene \\
& & \\
\hline
\end{tabular}




\begin{tabular}{|c|c|c|}
\hline Yeast strain & Genotype & Reference \\
\hline \multirow{2}{*}{ BY4741 } & Mat a, his3- $\Delta 1$ leu2 $\Delta 0$ met $15 \Delta 0$ ura3 $\Delta 0$ & Sikorski and \\
\hline & & 1989) \\
\hline $\operatorname{mic} 10 \Delta$ & $\begin{array}{l}\text { Mat a, his3- } \Delta 1 \text { leu2 } \Delta 0 \text { met } 15 \Delta 0 \text { ura3 } \Delta 0 \text {; } \\
\text { mic10::kanMX4 }\end{array}$ & (Alkhaja et al., 2012) \\
\hline $\operatorname{mic} 10 \Delta$ & $\begin{array}{l}\text { Mat a, his3- } \Delta 1 \text { leu2 } \Delta 0 \text { met } 15 \Delta 0 \text { ura3 } \Delta 0 \\
\text { mic10::kanMX4 [pRS413] }\end{array}$ & (Alkhaja et al., 2012) \\
\hline Mic10 & $\begin{array}{l}\text { Mat a, his3- } \Delta 1 \text { leu2 } \Delta 0 \text { met } 15 \Delta 0 \text { ura3 } \Delta 0 \\
\text { mic10::kanMX4 [pRS413-MIC10] }\end{array}$ & This study \\
\hline Mic10 G50/52A & $\begin{array}{l}\text { Mat a, his3- } \Delta 1 \text { leu2 } \Delta 0 \text { met } 15 \Delta 0 \text { ura3 } \Delta 0 \\
\left.\text { mic10::kanMX4 [pRS } 413-M I C 10^{\mathrm{G} 50 / 52 \mathrm{~A}}\right]\end{array}$ & This study \\
\hline Mic10 ${ }^{A 62 C}$ & $\begin{array}{l}\text { Mat a, his3- } \Delta 1 \text { leu2 } \Delta 0 \text { met } 15 \Delta 0 \text { ura3 } \Delta 0 \\
\left.\text { mic10::kanMX4 [pRS413-MIC } 10^{\mathrm{A} 62 \mathrm{C}}\right]\end{array}$ & This study \\
\hline $\operatorname{Mic} 10^{A 5 C}$ & $\begin{array}{l}\text { Mat a, his3- } \Delta 1 \text { leu2 } \Delta 0 \text { met } 15 \Delta 0 \text { ura3 } \Delta 0 \\
\left.\text { mic10::kanMX4 [pRS } 413-\mathrm{MIC} 0^{\mathrm{A5C}}\right]\end{array}$ & This study \\
\hline Mic $10^{\mathrm{V} 79 \mathrm{C}}$ & 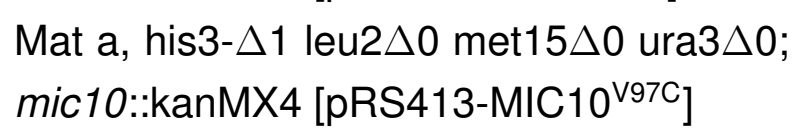 & This study \\
\hline $\operatorname{Mic} 10^{\mathrm{A} 5 \mathrm{C} / \mathrm{V} 97 \mathrm{C}}$ & $\begin{array}{l}\text { Mat a, his3- } \Delta 1 \text { leu2 } \Delta 0 \text { met } 15 \Delta 0 \text { ura3 } \Delta 0 \\
\text { mic10::kanMX4 [pRS413-MIC } 10^{A 5 C / N 97 C} \text { ] }\end{array}$ & This study \\
\hline
\end{tabular}




\subsubsection{Plasmids}

All plasmids used in this study were isolated from E.coli XL1 Blue cells and are listed in Table 3.7 and Table 3.8.

Table 3.7: E.coli plasmids

\begin{tabular}{|c|c|c|c|c|}
\hline Plasmid & Vector & Insert & $\begin{array}{l}\text { Selection } \\
\text { marker }\end{array}$ & Reference or source \\
\hline MM-55 & pGEX-4T-2 & $\begin{array}{l}\text { rat endophilinA1 } \\
\text { N247 (BAR) }\end{array}$ & Amp & AG Meinecke \\
\hline MM-86 & pET-28a(+) & Mic10 & Kan & This study \\
\hline MM-87 & pET10N & Tim23 & Apm & (Truscott et al., 2001) \\
\hline MM-115 & pPROEX HTc & Mic10 & Apm & This study \\
\hline MM-123 & pPROEX HTc & Mic10 $10^{G 8 A}$ & Apm & This study \\
\hline MM-124 & pPROEX HTc & Mic10G72A & Apm & This study \\
\hline MM-125 & pPROEX HTc & Mic10G74A & Apm & This study \\
\hline MM-126 & pPROEX HTc & Mic10 G70A & Apm & This study \\
\hline MM-127 & pPROEX HTc & Mic10 G78A & Apm & This study \\
\hline MM-128 & pPROEX HTc & Mic10G50A & Apm & This study \\
\hline MM-129 & pPROEX HTc & Mic10G52A & Apm & This study \\
\hline MM-130 & pPROEX HTc & Mic10G78A & Apm & This study \\
\hline MM-131 & pPROEX HTc & Mic10 G48/50A & Apm & This study \\
\hline MM-132 & pPROEX HTc & Mic 10 G48/52A & Apm & This study \\
\hline MM-133 & pPROEX HTc & Mic10G48/50/52A & Apm & This study \\
\hline MM-134 & pPROEX HTc & Mic $10^{G 50 / 52 A}$ & Apm & This study \\
\hline MM-135 & pPROEX HTc & Mic10 G72/74A & Apm & This study \\
\hline MM-138 & pPROEX HTc & Mic10 105 & Apm & This study \\
\hline MM-139 & pPROEX HTc & Mic10V97C & Apm & This study \\
\hline MM-144 & pPROEX HTc & Mic10 $10^{\mathrm{R} 62 \mathrm{C}}$ & Apm & This study \\
\hline MM-145 & pPROEX HTc & Mic10 A5C/V97C & Apm & This study \\
\hline MM-157 & pPROEX HTc & Mic10 A5C/G50/52A & Apm & This study \\
\hline MM-158 & pPROEX HTc & Mic10A5C/G72/74A & Apm & This study \\
\hline
\end{tabular}


Table 3.8: Yeast plasmids

\begin{tabular}{lllll}
\hline Plasmid & Vector & Insert & $\begin{array}{l}\text { Selection } \\
\text { marker }\end{array}$ & Source \\
\hline MM-147 & pRS413 & Mic10 & HIS3/Amp & This study \\
MM-148 & pRS413 & Mic10G50/52A & HIS3/Amp & This study \\
MM-156 & pRS413 & Mic10 ${ }^{\text {A62C }}$ & HIS3/Amp & This study \\
MM-166 & pRS413 & Mic10A5C & HIS3/Amp & This study \\
MM-170 & pRS413 & Mic10 $10^{\text {V79C }}$ & HIS3/Amp & This study \\
MM-173 & pRS413 & Mic10 $10^{\text {A5C/V97C }}$ & HIS3/Amp & This study
\end{tabular}

\subsubsection{Instruments and software}

The specific laboratory instruments used in this study are listed in Table 3.9. The software for data processing and image analysis are listed in Table 3.10 .

Table 3.9: Instruments and their manufacturers

\begin{tabular}{ll}
\hline Instrument & Manufacturer \\
\hline ÄKTAprime plus & GE Healthcare \\
Carbon-coated grids & Agar Scientific \\
CAWOMAT developing machine & CAWO Photochemisches Werk GmbH \\
(Centrifuge) 5415 R & Eppendorf \\
(Centrifuge) 5424 & Eppendorf \\
(Centrifuge) 5810 R & Eppendorf \\
(Centrifuge) F45-24-11 & Eppendorf \\
(Centrifuge) Optima MAX-XP & Beckman Coulter \\
(Centrifuge) Sorvall RC 6 Plus & Thermo Scientific \\
(Centrifuge) Sorvall RC 12BP & Thermo Scientific \\
(Centrifuge) TL-100 & Beckman Coulter \\
Desiccator & Vacuubrand \\
Dialysis membrane standard RC tubing & Spectrum Laboratories, Inc. \\
EmulsiFlex C5 homogenizer & Avestin \\
\hline
\end{tabular}




\begin{tabular}{|c|c|}
\hline Instrument & Manufacturer \\
\hline F-7000 fluorometer & Hitachi \\
\hline Filters PVDF & Roth \\
\hline GSTrap FF & GE Healthcare \\
\hline Hamilton syringe & Avanti polar lipids \\
\hline HisTrap HP $1 \mathrm{ml}$ and $5 \mathrm{~mL}$ & GE Healthcare \\
\hline HisTrap FP $1 \mathrm{ml}$ and $5 \mathrm{~mL}$ & GE Healthcare \\
\hline iMark Microplate Absorbance reader & Bio-Rad \\
\hline Nuclepore Track-Etch membrane & Whatman \\
\hline OSMOMAT 3000 (osmometer) & Gonotech \\
\hline PD MidiTrap G-25 & GE Healthcare \\
\hline Potter S (Dounce homogenisator) & Sartorius \\
\hline (Rotor) JA-10 & Beckman Coulter \\
\hline (Rotor) JA-20 & Beckman Coulter \\
\hline (Rotor) Sorvall F10S-6x500Y & Thermo Scientific \\
\hline (Rotor) Sorvall F14S-6x250Y & Thermo Scientific \\
\hline (Rotor) Sorvall H-1200 & Thermo Scientific \\
\hline (Rotor) Sorvall SS-34 & Thermo Scientific \\
\hline (Rotor) SW $60 \mathrm{Ti}$ & Beckman Coulter \\
\hline (Rotor) TLA-45 & Beckman Coulter \\
\hline (Rotor) TLA-55 & Beckman Coulter \\
\hline (Rotor) TLA-100.3 & Beckman Coulter \\
\hline (Rotor) TYPE $75 \mathrm{Ti}$ & Beckman Coulter \\
\hline Sonicator ( cell disruptor W-220F) & Heat systems - ultrasonics Inc. \\
\hline SpeedVac concentrator & Savant \\
\hline Superdex 75 16/600 gel-filtration column & GE Healthcare \\
\hline Superdex 200 16/600 gel-filtration column & GE Healthcare \\
\hline Thermomixer comfort & Eppendorf \\
\hline TPersonal 48 thermocycler & Biometra \\
\hline Transmission electron microscopy & JOEL, Ltd. \\
\hline (TEM) JOEL JEM-1011 & \\
\hline
\end{tabular}

continued... 
...continued

Instrument

Manufacturer

Transmission electron microscopy

FEI Europe

Philips CM 120

Ultraviolet (UV) solo (UV documentation) Biometra

Vesicle Prep Pro

Nanion Technoologies

Zetasizer Nano S

Malven Instruments $\mathrm{GmbH}$

Table 3.10: Softwares and their manufacturers

\begin{tabular}{ll}
\hline Software & Manufacturer \\
\hline Adobe Illustrator CS5.1 & Adobe Systems \\
Adobe Photoshop CS5.1 & Adobe Systems \\
ImageJ & Wayne Rasband \\
Microsoft office 2010 & Microsoft Corporation \\
OriginPro 8.5G & OriginLab Corporation \\
Serial cloner & SerialBasics \\
UNICORN & GE Healthcare
\end{tabular}

\subsection{Methods}

\subsubsection{Cultivation and application of biological material}

\subsubsection{E.coli growth}

BL21 and XL1 Blue E.coli strains were grown on LB - lysogeny broth (1 \% $\mathrm{NaCl}, 0.5$ $\%$ yeast extract, $1 \%$ tryptone) media. Solid media was supplemented with $15 \mathrm{~g} / \mathrm{L}$ agar. Antibiotic selection markers ampicillin and kanamycin were used in concentrations $100 \mathrm{mg} / \mathrm{L}$ and $30 \mathrm{mg} / \mathrm{mL}$, respectively. Cells containing appropriate antibioticresistant gene were grown at $37^{\circ} \mathrm{C}$ at $180 \mathrm{rpm}$.

For nuclear magnetic resonance (NMR) studies the cells were grown on M9 
minimal media (M9 salts - $0.6 \% \mathrm{Na}_{2} \mathrm{HPO}_{4}, 0.3 \% \mathrm{KH}_{2} \mathrm{PO}_{4}, 0.1 \% \mathrm{NaCl}, 0.1 \%$ $\left({ }^{15} \mathrm{NH}_{4}\right)_{2} \mathrm{SO}_{4} ; 0.2 \%$ D-Glucose- ${ }^{12} \mathrm{C} ; 0.024 \% \mathrm{MgSO}_{4} ; 20 \mathrm{~mL} / \mathrm{L}$ trace metal solution $\left(0.01 \% \mathrm{ZnSO}_{4}-7 \mathrm{H}_{2} \mathrm{O}, 0.08 \% \mathrm{MnSO}_{4}-\mathrm{H}_{2} \mathrm{O}, 0.05 \% \mathrm{FeSO}_{4}-7 \mathrm{H}_{2} \mathrm{O}, 0.01 \% \mathrm{CuSO}_{4}, 0.1\right.$ $\% \mathrm{CaCl}_{2} ; 1 \% \mathrm{v} / \mathrm{v} \mathrm{MeM}$ ) in the presence of appropriate antibiotic at $30^{\circ} \mathrm{C}, 180 \mathrm{rpm}$.

\subsubsection{Preparation and transformation of chemically competent E.coli cells}

Chemically competent E.coli cells were prepared according to previously described method (Hanahan, 1983). To this end, $1 \mathrm{~L}$ culture (without an antibiotic) of BL21 or XL1 Blue cells were grown to an OD (optical density) of 0.6-0.7 ( Abs $_{600 \mathrm{~nm}}$ ) and chilled on ice for $15 \mathrm{~min}$. The cells were collected by centrifugation for $10 \mathrm{~min}$ at $2700 \mathrm{xg}$ and resuspended in $200 \mathrm{~mL}$ ice-cold buffer 1 (30 mM KAc, $100 \mathrm{mM} \mathrm{RbCl}$, $10 \mathrm{mM} \mathrm{CaCl}_{2}, 50 \mathrm{mM} \mathrm{MnCl}_{2}, 15 \%$ glycerol, pH 5.8). The cells were further pelleted and resuspended in $30 \mathrm{~mL}$ buffer 2 (10 mM MOPS, $10 \mathrm{mM} \mathrm{RbCl}, 75 \mathrm{mM} \mathrm{CaCl}_{2}, 15 \%$ glycerol, $\mathrm{pH}$ 6.5), aliquoted into $110 \mu \mathrm{L}$ and after rapid freezing in liquid nitrogen were stored at $-80^{\circ} \mathrm{C}$. For transformation $50 \mu \mathrm{L}$ cells thawed on ice were mixed with 100 ng plasmid DNA or $5 \mu \mathrm{L}$ ligation or site-directed mutagenesis PCR product mix (see 3.2.2.2 and 3.2.2.3 and incubated on ice for $5 \mathrm{~min}$. Transformation was achieved by heat-shock for $45 \mathrm{sec}$ at $42{ }^{\circ} \mathrm{C}$. The cells were recovered by shaking in $300 \mu \mathrm{L} \mathrm{LB}$ media and were ultimately transferred to an appropriate antibiotic selection plate.

\subsubsection{Preparation of E.coli whole cell lysate}

To test the expression success of recombinant proteins in E.coli cells, the protein expression levels were tested at different experimental conditions. Briefly, $30 \mathrm{~mL}$ culture of BL21(DE3) cells containing a plasmid with the gene of interest was grown to $\mathrm{OD}$ of 0.6 and $1.5 \mathrm{OD}$ (amount to take $(\mathrm{mL})=O D_{\text {interest }} / \mathrm{OD}_{\text {measured }}$ ) was taken as an non-induced sample before inducing protein expression by adding either $0.5 \mathrm{mM}$ and $1 \mathrm{mM}$ IPTG. The induced cells were collected by taking $1.5 \mathrm{OD}$ sample every hour consecutively within 3 hours. The cells were collected for $5 \mathrm{~min}$ at $2300 \mathrm{xg}$. The pellets were resuspended in $400 \mu \mathrm{L}$ sample loading buffer and the resuspended cells were lysed by using a sonicator (three times $45 \mathrm{sec}$ ). The protein overexpression 
success was monitored by subjecting the whole cell lysates to SDS-PAGE analysis (see section 3.2.3.1).

\subsubsection{S. cerevisiae growth}

Yeast cells from Table 3.6 were grown at $30{ }^{\circ} \mathrm{C}$ in selective medium $(0.67 \%$ yeast nitrogen base without amino acids, $0.07 \%$ complete supplement mixture lacking histidine amino acid) contained either $2 \%$ glucose (SD), $3 \%$ glycerol (SG) or $3 \%$ lactate (SLac) by shaking at $120 \mathrm{rpm}$. The plates were supplemented by $2.5 \%$ agar. The growth analysis of different yeast strains was carried out by serial dilutions of cells were spotted on SD, SG or SLac plates at $18,30,37^{\circ} \mathrm{C}$ temperature for a couple of days. For long term storage glycerol cryo-stocks were prepared by mixing equal volumes of $30 \%$ glycerol to liquid culture and freezing at $-80{ }^{\circ} \mathrm{C}$.

\subsubsection{Preparation and transformation of chemically competent $S$. cerevisiae cells}

S. cerevisiae competent cells were prepared according to the previously described method (Gietz and Woods, 2002). Briefly, $50 \mathrm{~mL}$ yeast culture of 2x YPAD (1\% yeast extract, $2 \%$ peptone, $10 \%$ adenine hemisulfate, $2 \%$ glucose) were grown to $\mathrm{OD}_{600} 1.5-2.0$ at $30{ }^{\circ} \mathrm{C}, 120 \mathrm{rpm}$. The cells were collected by centrifugation for 5 $\mathrm{min}$, at $5000 \mathrm{xg}$, at $4{ }^{\circ} \mathrm{C}$ and washed with $10 \mathrm{~mL}$ sterile water. After centrifugation for $5 \mathrm{~min}$, at $5000 \mathrm{xg}$, at $4{ }^{\circ} \mathrm{C}$ the cells were resuspended in $10 \mathrm{~mL}$ LiAc buffer $(0.1$ M LiAc, $5 \mathrm{mM}$ Tris, $0.1 \mathrm{mM}$ EDTA, $\mathrm{pH} 8.0$ ), incubated for $30 \mathrm{~min}$ at $4{ }^{\circ} \mathrm{C}$ with gentle agitation and subsequently were collected by centrifugation at $5000 \mathrm{xg}$, at $4{ }^{\circ} \mathrm{C}$. The pellet was resuspended in $500 \mu \mathrm{L}$ LiAc buffer and aliquoted into $100 \mu \mathrm{L}$ volumes. The competent cells were either stored at $-80{ }^{\circ} \mathrm{C}$ or used immediately for plasmid transformation. For plasmid transformation, $100 \mu \mathrm{L}$ competent cells were incubated with $500 \mathrm{ng}$ appropriate plasmid DNA and $100 \mu \mathrm{g}$ herring sperm DNA (denatured for $5 \mathrm{~min}$ at $100{ }^{\circ} \mathrm{C}$ ) for $30 \mathrm{~min}$ at $30^{\circ} \mathrm{C}$ with gentle agitation. Afterwards, $600 \mu \mathrm{L} 40$ $\%$ PEG 4000 in LiAc buffer was added and the cells were incubated for $1 \mathrm{~h}$ at $30{ }^{\circ} \mathrm{C}$ under shaking at $1000 \mathrm{rpm}$. The cells were supplemented by $68 \mu \mathrm{L}$ sterile DMSO and mixed to homogeneity by inverting the tube. Ultimately, the cells were incubated 
for $15 \mathrm{~min}$ at $42^{\circ} \mathrm{C}$ and spun down for $1 \mathrm{~min}$ at $400 \mathrm{xg}$. The cells were resuspended into $200 \mu \mathrm{L}$ sterile water, transfered onto the selective plates and incubated for 2 days at $30^{\circ} \mathrm{C}$.

\subsubsection{Isolation of mitochondria form S. cerevisiae cells}

Crude mitochondria were isolated according to the previously described method (Meisinger et al., 2006). To this end, the cells from 3.2.1.5 were grown to $\mathrm{OD}_{600}$ of 1.5 in SG selective media (see 3.2.1.4) and collected by centrifugation for $10 \mathrm{~min}$ at $7000 \mathrm{xg}$ at $18^{\circ} \mathrm{C}$. The pellet was washed with sterile water and harvested by centrifugation for $10 \mathrm{~min}$ at $2700 \mathrm{xg}$ at $18^{\circ} \mathrm{C}$. The cells were resuspended with $2 \mathrm{~mL}$ DTT buffer (100 mM Tris, $10 \mathrm{mM} \mathrm{DTT,} \mathrm{pH} \mathrm{9.4)} \mathrm{per} \mathrm{gram} \mathrm{cells} \mathrm{(wet} \mathrm{weight)} \mathrm{and} \mathrm{incubated}$ for $30 \mathrm{~min}$ at $30{ }^{\circ} \mathrm{C}$ by shaking at $220 \mathrm{rpm}$. After collecting the cells by centrifugation for $8 \mathrm{~min}$ at $2700 \mathrm{xg}$ at $18{ }^{\circ} \mathrm{C}$ the cells were washed with $200 \mathrm{~mL} 1.2 \mathrm{M}$ sorbitol. Following the centrifugation for $8 \mathrm{~min}$ at $2700 \mathrm{xg}$ at $18^{\circ} \mathrm{C}$, the pellet was resuspended in $100 \mathrm{~mL}$ zymolyase buffer (20 mM KPi, $1.2 \mathrm{M}$ sorbitol, $\mathrm{pH} 7.4$ ) containing $4 \mathrm{mg} / \mathrm{g}$ zymolyase. The mixture was incubated for $90 \mathrm{~min}$ at $30{ }^{\circ} \mathrm{C}$ by shaking at $220 \mathrm{rpm}$ followed by $10 \mathrm{~min}$ centrifugation at $1500 \mathrm{xg}$ at $18{ }^{\circ} \mathrm{C}$. The pellet was washed with $100 \mathrm{~mL}$ zymolyase buffer and collected for $10 \mathrm{~min}$ at $1500 \mathrm{xg}$ at $18{ }^{\circ} \mathrm{C}$. Afterwards the pellets were resuspended in $7 \mathrm{~mL} / \mathrm{g}$ ice-cold homogenization buffer (10 $\mathrm{mM}$ Tris, $600 \mathrm{mM}$ sorbitol, $1 \mathrm{mM}$ EDTA, $1 \mathrm{mM}$ PMSF, $2 \mathrm{~g} / \mathrm{L}$ fatty-acid free BSA, $\mathrm{pH}$ 7.4). The suspension was processed twenty times by using Dounce homogenizer at $750 \mathrm{rpm}$ on ice. The homogenate was centrifuged for $5 \mathrm{~min}$ at $1500 \mathrm{xg}$ at $4{ }^{\circ} \mathrm{C}$. The pellet was discarded and the supernatant was centrifuged for $10 \mathrm{~min}$ at $2700 \mathrm{xg}$ at $4{ }^{\circ} \mathrm{C}$. The mitochondria were collected by centrifugation for $15 \mathrm{~min}$ at $17000 \mathrm{xg}$ at $4{ }^{\circ} \mathrm{C}$. The mitochondrial pellet was resuspended in $10 \mathrm{~mL}$ SEM buffer $(250 \mathrm{mM}$ sucrose, $1 \mathrm{mM}$ EDTA, $10 \mathrm{mM}$ MOPS pH 7.2 ) containing 0.5-1.0 mM PMSF and centrifuged for $15 \mathrm{~min}$ at $17000 \mathrm{xg}$ at $4{ }^{\circ} \mathrm{C}$. The washed pellet was resuspended in $600 \mu \mathrm{L} \mathrm{SEM}$ buffer. Mitochondrial protein concentration was determined by Bradford protein determination assay using Bio-Rad and a IgG protein standard at 7.5, 15, 30 and 60 $\mu \mathrm{L}$ concentrations. Absorption shift was measured at $595 \mathrm{~nm}$ and the concentration of mitochondria was set to $10 \mu \mathrm{g} / \mu \mathrm{L}$. Ultimately, the isolated mitochondria were aliquoted into $50 \mu \mathrm{L}$ volumes, frozen in liquid nitrogen and stored at $-80{ }^{\circ} \mathrm{C}$. 


\subsubsection{Molecular biology}

\subsubsection{Plasmid DNA isolation from E.coli cells}

For plasmid isolation "Wizard Plus SV Minipreps DNA Purification System" (Promega) was used and the isolation steps were carried out according to the manufacturers instructions. Briefly, XL1 Blue cells grown in $5 \mathrm{~mL}$ LB medium were collected by centrifugation for $7 \mathrm{~min}$ at $16000 \mathrm{xg}$. The pellet was thoroughly resuspended in 250 $\mu \mathrm{L}$ resuspension buffer, followed by addition of $250 \mu \mathrm{L}$ cell lysis buffer. The solution was mixed carefully, $10 \mu \mathrm{L}$ alkaline protease solution was added to it and incubated for $5 \mathrm{~min}$ at room temperature. After addition of $350 \mu \mathrm{L}$ neutralization buffer and careful mixing, the mixture was centrifuged for $10 \mathrm{~min}$ at $16000 \mathrm{xg}$ at room temperature. The supernatant was transfered to the filter column and centrifuged for $1 \mathrm{~min}$ at $16000 \mathrm{xg}$. The filter column was washed by $750 \mu \mathrm{L}$ and $250 \mu \mathrm{L}$ washing buffer by consecutive centrifugation for $1 \mathrm{~min}$ and $2 \mathrm{~min}$ at $16000 \mathrm{xg}$. The plasmid DNA was eluted by addition of $50 \mu \mathrm{L}$ nuclease-free water and subsequent centrifugation for 1 $\min$ at $16000 \mathrm{xg}$. The isolated plasmid was stored at $-80{ }^{\circ} \mathrm{C}$.

\subsubsection{Polymerase chain reaction (PCR)}

The desired DNA fragments were amplified either form yeast genomic (AG Rehling) and plasmid DNA by polymerase chain reaction. The PCR reaction mixture (50 $\mu \mathrm{L})$ contained, $100 \mathrm{ng}$ template DNA, $0.3 \mu \mathrm{M}$ forward and reverse primers, $0.2 \mu \mathrm{M}$ dNTP mix, $3 \%$ DMSO, 1x Phusion HF buffer and $1 U$ Phusion DNA polymerase. The cycling conditions were set to $3 \mathrm{~min}$ at $98{ }^{\circ} \mathrm{C}$ for initial denaturation as well as for enzyme activation, followed by 35 cycles of consecutive steps of $10 \mathrm{sec}$ at $98{ }^{\circ} \mathrm{C}$ denaturation, $30 \mathrm{sec}$ at $55^{\circ} \mathrm{C}$ for primer annealing and $30 \mathrm{sec}$ at $72{ }^{\circ} \mathrm{C}$ for chain elongation. For site-directed mutagenesis, PCR product was digested by 1 $\mu \mathrm{L}$ Dpnl. The sample was transformed into XL1 Blue cells as described in 3.2.1.2. For cloning PCR product was analyzed by agarose gel electrophoresis ( $1 \%$ agarose (Roth) dissolved in $40 \mathrm{mM}$ Tris, $20 \mathrm{mM}$ acetic acid, $1 \mathrm{mM}$ EDTA buffer) at $120 \mathrm{~V}$ for 30-45 min. PCR products were cleaned up by using the Wizard SV Gel and PCR CleanUp System (Promega). Briefly, the PCR amplification was mixed with the equal volume of membrane binding solution and transfered to the filter columns. Following 
1 min incubation the sample was spun down for $1 \mathrm{~min}$ at $16000 \mathrm{xg}$. The filter was washed with $700 \mu \mathrm{L}$ and $500 \mu \mathrm{L}$ washing buffer by centrifugation for 1 min and 5 $\min$ at $16000 \mathrm{xg}$. The DNA was eluted by addition of $50 \mu \mathrm{L}$ nuclease-free water and subsequent centrifugation for $1 \mathrm{~min}$ at $16000 \mathrm{xg}$. The eluate was either further used or was stored at $-20{ }^{\circ} \mathrm{C}$.

\subsubsection{Cloning}

Both, the amplified and purified insert from 3.2.2.2 and the vector of interest were digested with the appropriate restriction endonucleases (Thermo Scientific). To this end, $20 \mu \mathrm{L}$ reaction mixture (1 $\mu \mathrm{g}$ circular vector, $2 \mathrm{U}$ (unit) BamH1, 4U Sal1, 1x BamH1 buffer) for Vector and $40 \mu \mathrm{L}$ (200 ng insert, 4U BamH1, 8U Sal1, 1x BamH1 buffer ) for insert were incubated at $37^{\circ} \mathrm{C}$ for $1.5 \mathrm{~h}$. The digests were separated by agarose gel electrophoresis (see 3.2.2.2), followed by excision of the appropriate DNA bands. The digested DNA was extracted from the agarose gel by using Wizard SV Gel and PCR CleanUp System. Briefly, the gel slices were hydrated with an equal $w / v$ amount of membrane binding solution and dissolved by incubating at $50-60{ }^{\circ} \mathrm{C}$ and vortexing. The following purification steps are described in section 3.2.2.2. For ligation T4 DNA Ligase (Thermo Scientific) was used. Briefly, $20 \mu \mathrm{L}$ ligation reaction mixture (50 ng linear vector, 1x T4 DNA Ligase buffer, 1.5U T4 DNA Ligase and different insert amounts according to vector:insert molar ratios $1: 1 ; 1: 3 ; 1: 5)$ was incubated for $18 \mathrm{~h}$ at $16{ }^{\circ} \mathrm{C}$. The $5 \mu \mathrm{L}$ of each sample was transfered to $50 \mu \mathrm{L}$ competent XL1 Blue cells (see 3.2.1.2).

\subsubsection{DNA sequencing}

All the DNA samples were sequenced by GATC Biotech (Constance, Germany).

\subsubsection{Protein biochemistry}

\subsubsection{SDS-PAGE}

Sodium dodecyl sulfate polyacrylamide gel electrophoresis (SDS-PAGE) was employed for protein separation. Separation gels had a composition of 10-15\%30\% 
/ $0.8 \%$ acrylamid / bis-acrylamid, $386 \mathrm{mM}$ Tris/ $\mathrm{HCl} \mathrm{pH} \mathrm{8.8,} 0.1 \%$ SDS, $0.1 \%$ APS, $0.08 \%$ TEMED. The stacking gel consisted of $5 \% 30 \% / 0.8 \%$ acrylamid / bisacrylamid, $126 \mathrm{mM}$ Tris/HCl pH 6.8, $0.1 \%$ SDS, $0.1 \%$ APS, $0.2 \%$ TEMED. The running (Laemmli) buffer contained $25 \mathrm{mM}$ Tris, $191 \mathrm{mM}$ glycine, $0.1 \%$ SDS. The vertical gel electrophoresis was carried out in the MINI-Protein II (Bio-Rad) system at constant $180 \mathrm{~V}$ run conditions. NuPAGE precast gel (Novex) was run in Novex Mini cell (Invitrogen) gel electrophoresis system according to the manufacturers instruction. As running buffer MES buffer system was used. In both gel electrophoresis system the sample loading buffer contained $2 \%$ SDS, $10 \%$ glycerol, $60 \mathrm{mM}$ Tris/ $\mathrm{HCl} \mathrm{pH} \mathrm{6.8,} 1 \% \beta$-mercaptoethanol, $0.01 \%$ bromphenol blue. The Mark12 unstained (Novex) or PageRuler prestained (Thermo Scientific) protein markers were used as a molecular weight standard.

\subsubsection{BN-PAGE}

For blue native PAGE (BN-PAGE) analysis approximately $2.5 \mu \mathrm{g}$ Mic10 and its variants dissolved in $0.05 \%$ or $0.1 \%$ DDM (Mic10 WT also was dissolved in $1 \%$ digitonin containing buffer) containing buffer were mixed with 20:1 v/v $5 \%$ Coomassie G 250 and NativePAGE sample buffer and centrifuged for $3 \mathrm{~min}$ at $16000 \mathrm{xg}$ at $4{ }^{\circ} \mathrm{C}$. The supernatants were loaded on the NativePAGE 3-12 \% or 4-16\% Bis-Tris gels and the run was performed at a constant $150 \mathrm{~V}$. The anode buffer contained $50 \mathrm{mM}$ BisTris, $\mathrm{pH}$ 7.0. The cathode buffer contained $50 \mathrm{mM}$ Tricine, $15 \mathrm{mM}$ Bis-Tris, with or without $0.02 \%$ Coomassie G 250. One third of the electrophoresis was performed in the presence of the blue cathode buffer and the rest in the presence of the light cathode buffer. The gels were subsequently analyzed by coomassie staining. Either HMW calibration kit (GE Healthcare) and NativeMark unstained protein standard were used as molecular weight markers.

\subsubsection{Coomassie Brilliant Blue staining}

Following the gel electrophoresis the gels from 3.2.3.1 and 3.2.3.2 as well as membranes after western blotting from 3.2.3.4 were stained with Coomassie Brilliant Blue staining. The stain contained $25 \%$ ethanol, $10 \%$ acetic acid and $0.1 \%$ Coomassie 
Brilliant Blue R 250. Destainer had a same composition as stainer but without Coomassie Brilliant Blue R 250.

\subsubsection{Western blot}

For western blotting either semi-dry (Peqlab) and wet transfer (Bio-Rad) systems were employed. The blotting buffer for semi-dry transfer contained $20 \mathrm{mM}$ Tris, 150 mM glycin, $0.02 \%$ SDS, $20 \%$ methanol. For wet transfer the buffer composition was 25 mM Tris, $192 \mathrm{mM}$ glycine, $0.1 \%$ SDS, $20 \%$ methanol, pH 8.3. PVDF membrane was briefly activated in methanol. Fiber pads (only for wet transfer), blotting papers (two on each side of the sandwich), PVDF membrane and the gel were soaked in an appropriate blotting buffer. For semi-dry transfer system blotting was performed at $13 \mathrm{~V}, 125 \mathrm{~mA}$ for $1 \mathrm{~h}$, whereas for wet transfer system at constant $100 \mathrm{~V}, 350 \mathrm{~mA}$ for $1 \mathrm{~h}$ in the presence of a cooling unit.

\subsubsection{Immunodetection}

Following blotting the PVDF membrane was blocked in TBS-T buffer (20 mM Tris, $125 \mathrm{mM} \mathrm{NaCl}, 0.1 \%$ Tween 20, $\mathrm{pH}$ 7.5) containing $5 \%$ milk powder for $1 \mathrm{~h}$ at room temperature. Afterwards the membrane was briefly rinsed with TBS-T and incubated in an appropriate primary antibody containing solution (all the primary antibodies but $\alpha$-His were dissolved in TBS-T containing $5 \%$ milk, $\alpha$-His antibody was dissolved in TBS-T) and incubated for 45-60 min at room temperature or $16 \mathrm{~h}$ at $4{ }^{\circ} \mathrm{C}$. The membrane was washed two times for 15 min with TBS-T buffer and incubated in an appropriate secondary antibody (HRP coupled, dilutions 1:10000) containing solution for 45-60 min at room temperature. The membrane was washed with TBS-T buffer two times for $15 \mathrm{~min}$. The ECL or Luminata solutions were added to the membrane and signals were detected on $x$-ray films by developing in the CAWOMAT developing machine. 


\subsubsection{Recombinant protein affinity-tag purification from E.coli}

Recombinantly expressed proteins in E.coli were purified according to the previously described methods (Truscott et al., 2001; Meinecke et al., 2013). E.coli growth conditions and protein overexpression test are described in 3.2.1.1 and 3.2.1.3, respectively.

Isolation of inclusion bodies from E.coli - after harvesting the induced E.coli cells for $20 \mathrm{~min}$ at $7000 \mathrm{xg}$ at $4{ }^{\circ} \mathrm{C}$, the bacterial pellet was resuspended in $10 \mathrm{~mL}$ lysis buffer (100 mM NaCl, $50 \mathrm{mM}$ Tris, $\mathrm{pH} 8.0$ ) containing $0.1 \mathrm{mg} / \mathrm{mL}$ lysozyme, $0.5 \mathrm{mM}$ PMSF, $10 \mu \mathrm{g} / \mathrm{mL}$ Dnase I, 1 pill/50 $\mathrm{mL}$ protease inhibitor cocktail (EDTA-free) per gram wet cell weight. The cells were lysed by passing the cell suspension three times through an EmulsiFlex-C5 homogenizer (Avestin) at 15000-17000psi/103-117 $\mathrm{MPa}$. Afterwards, $4 \mathrm{mg} / \mathrm{g}$ deoxycholic acid and $10 \mu \mathrm{g} / \mathrm{mL}$ Dnase I were added to the cell lysate and incubated for $30 \mathrm{~min}$ at room temperature under mild stirring. The cell lysate was centrifuged for $1 \mathrm{~h}$ at $4200 \mathrm{xg}$ at $4{ }^{\circ} \mathrm{C}$. The pellet was resuspended in $100 \mathrm{~mL}$ triton buffer (100 mM NaCl, $50 \mathrm{mM}$ Tris, $1 \mathrm{mM}$ EDTA, $10 \mathrm{mM}$ DTT, $2 \%$ Triton X-100, $\mathrm{pH} 8.0$ ) and stirred for $30 \mathrm{~min}$ on ice. The suspension was centrifuged for $30 \mathrm{~min}$ at $4200 \mathrm{xg}$ at $4{ }^{\circ} \mathrm{C}$, the pellet was resuspended in $100 \mathrm{~mL}$ buffer $(100 \mathrm{mM}$ $\mathrm{NaCl}, 50 \mathrm{mM}$ Tris, $1 \mathrm{mM}$ EDTA, $10 \mathrm{mM}$ DTT, pH 8.0) and stirred for $30 \mathrm{~min}$ on ice. Following centrifugation the pellet was resuspended in $100 \mathrm{~mL}$ lysis buffer and the inclusion bodies were collected by centrifugation for $30 \mathrm{~min}$ at $4200 \mathrm{xg}$ at $4{ }^{\circ} \mathrm{C}$. The purified inclusion bodies were either stored at $-20{ }^{\circ} \mathrm{C}$ or immediately subjected to affinity tag purification. For isolation of recombinantly expressed His-tagged protein, the inclusion bodies were dissolved in $3 \mathrm{~mL} / \mathrm{g}$ urea or GuHCl buffer (for Mic10 and it's mutant forms $8 \mathrm{M}$ urea/6 M GuHCl, $150 \mathrm{mM} \mathrm{NaCl}, 10 \mathrm{mM}$ Tris, $40 \mathrm{mM}$ imidazole, 2 mM DTT, pH 8.0 and for Tim23 $6 \mathrm{M}$ urea, $150 \mathrm{mM} \mathrm{NaCl}, 10 \mathrm{mM}$ Tris, $40 \mathrm{mM}$ imidazole, $\mathrm{pH} 8.0$ ) and stirred for $60 \mathrm{~min}$ at room temperature. After centrifugation for $20 \mathrm{~min}$ at $17400 \mathrm{xg}$ at room temperature, the soluble supernatant was filtered through a PVDF filter (Roth) with a pore size $0.45 \mu \mathrm{m}$. The filtered suspension was applied with $0.5-1.0 \mathrm{~mL} / \mathrm{min}$ flow rate to the pre-equilibrated $1 \mathrm{~mL}$ or $5 \mathrm{~mL}$ HisTrap

pre-packed column (GE Healthcare) in the urea or GuHCl buffer using ÄKTAprime plus (GE Healthcare). The column was washed twenty times column volume urea or $\mathrm{GuHCl}$ buffer and the protein was eluted by applying the urea or $\mathrm{GuHCl}$ buffer 
containing $500 \mathrm{mM}$ imidazole.

For small scale test protein isolations the batch affinity-tag purification was employed. For this purpose Ni-NTA Agarose beads with $0.5-1.0 \mathrm{~mL}$ bed volume was used. The protein purification was carried out in the above-mentioned buffer systems under gravity.

The eluted protein was further purified by employing size-exclusion chromatography described in 3.2.3.7.

Preparation of whole cell lysate of soluble proteins - the whole cell lysate of E.coli expressing a soluble protein GST-endophilin BAR or GST-PreScission protease were obtained similarly as described above but the lysis buffer composition was $150 \mathrm{mM}$ $\mathrm{NaCl}, 50 \mathrm{mM}$ HEPES, $2 \mathrm{mM}$ DTT, $2 \mathrm{mM}$ EDTA, pH 7.4. After opening the cells the lysate was first centrifuged for $20 \mathrm{~min}$ at $4200 \mathrm{xg}$ at $4{ }^{\circ} \mathrm{C}$, followed by $1 \mathrm{~h}$ centrifugation at $200,000 \mathrm{xg}$ at $4{ }^{\circ} \mathrm{C}$. The supernatant was filtered through a PVDF filter with a pore size $0.45 \mu \mathrm{m}$ and subjected to affinity tag purification. To this end, the filtered supernatant was loaded with $0.2 \mathrm{~mL} / \mathrm{min}$ flow rate to the pre-equilibrated 5 mL GSTrap pre-packed column (GE Healthcare) in the lysis buffer using ÄKTAprime plus. The column with bound protein was washed with twenty times column volume lysis buffer and the protein was eluted with the appropriate amount of elution buffer (50 mM Tris, $10 \mathrm{mM} \mathrm{L-glutathione} \mathrm{reduced).} \mathrm{The} \mathrm{GST} \mathrm{tag} \mathrm{from} \mathrm{GST-endophilin} \mathrm{BAR}$ was cleaved by using 1:50 w/w GST-PreScission protease:GST-endophilin BAR for $16 \mathrm{~h}$ at $4{ }^{\circ} \mathrm{C}$. Subsequently, the solution was applied to GSTrap and the flow through with endophilin BAR was collected. The sample was further purified to single-band purity by size-exclusion chromatography (see 3.2.3.7).

\subsubsection{Size-exclusion chromatography}

The isolated proteins from 3.2.3.6 were further purified using size-exclusion chromatography. The sample with $0.2,0.5,1,2$ or $5 \mathrm{~mL}$ volumes were applied to the gel-filtration column pre-equilibrated in at least 1 column volume desired buffer using ÄKTAprime plus. For Mic10 and Tim23 separation the Superdex 200 (16/600) (GE Healthcare) column was employed, whereas for endophilin BAR Superdex 75 (16/600) (GE Healthcare). The separation was carried out in appropriate buffer systems (Mic10 - $8 \mathrm{M}$ urea/6 M GuHCl, 150 mM NaCl, 10 mM Tris, 2 mM DTT, pH 8.0; Tim23 - $6 \mathrm{M}$ urea, $150 \mathrm{mM} \mathrm{NaCl}, 10 \mathrm{mM}$ Tris, $\mathrm{pH}$ 8.0; endophilin BAR - $150 \mathrm{mM}$ 
$\mathrm{NaCl}, 50 \mathrm{mM}$ HEPES, $\mathrm{pH}$ 7.4) with $1 \mathrm{~mL} / \mathrm{min}$ flow rate. The purification success was analyzed by SDS-PAGE and subsequent coomassie staining.

Since, $\mathrm{GuHCl}$ forms a precipitate with SDS when dissolved together, Mic10 samples in $6 \mathrm{M} \mathrm{GuHCl}$ containing buffer was TCA precipitated (see 3.2.3.8), then resuspended in SDS sample loading buffer and subjected to SDS-PAGE analysis.

\subsubsection{Protein precipitation by TCA}

In order to avoid the Mic10 sample precipitation in the presence of SDS and $\mathrm{GuHCl}$ (see 3.2.3.7) or reduce the sample volume for SDS-PAGE analysis (see 3.2.4.3 and 3.2.4.4 protein precipitation by trichloroacetic acid (TCA) was used. To this end, samples were incubated with ice-cold $10 \%$ TCA for 30 min on ice, followed by centrifugation for $30 \mathrm{~min}$ at $16000 \mathrm{xg}$ at $4{ }^{\circ} \mathrm{C}$. The residual TCA was removed by washing the pellets in $500 \mu \mathrm{L}$ ice-cold acetone followed by centrifugation for $30 \mathrm{~min}$ at 16000 $\mathrm{xg}$ at $4{ }^{\circ} \mathrm{C}$. The pellets were dried for $30 \mathrm{~min}$ in SpeedVac concentrator, resuspended in SDS sample loading buffer and subjected to SDS-PAGE analysis.

\subsubsection{Protein refolding in various mild detergents}

In order to refold the proteins isolated in harsh experimental conditions such as treating them by urea and $\mathrm{GuHCl}$ containing buffers, the protein samples were dialysed against mild detergent containing buffers. To this end, the $0.5-1.0 \mathrm{~mL}$ sample dissolved in $8 \mathrm{M}$ urea or $6 \mathrm{M} \mathrm{GuHCl}$ containing buffer was transfered into the $3.5 \mathrm{KDa}$ pore size dialysis tubing membrane (Spectrum Laboratories, Inc.) and stirred in $50 \mathrm{~mL}$ appropriate detergent containing buffer for $2 \mathrm{~h}$ at room temperature. Afterwards the buffer was exchanged with the same composition $50 \mathrm{~mL}$ fresh buffer and the membrane was dialysed under stirring for $16 \mathrm{~h}$ at $4{ }^{\circ} \mathrm{C}$. Detergents with low CMC (critical micelle concentration) form large molecular weight micelles and therefore cannot be dialysed. Therefore, detergents such as DDM and DM were added to the dialysis membrane in appropriate amounts. Subsequently, the sample was centrifuged for $45 \mathrm{~min}$ at $125,000 \mathrm{xg}$ at $4{ }^{\circ} \mathrm{C}$ and the supernatant and the precipitated protein were separated. The renatured sample was either immediately used or was frozed in liquid nitrogen and stored at $-20^{\circ} \mathrm{C}$. For protein solubility analysis 
the pellet was resuspended in $20 \mu \mathrm{L}$ sample loading buffer. The equal amounts of resuspended pellet and soluble protein solutions were loaded on SDS-PAGE and analyzed by subsequent coomassie staining.

\subsubsection{Protein labeling}

Mic10 labeling for fluorescence studies (see 3.2.4.6) was carried out by using Alexa Flour 488 Protein labeling kit (Invitrogen) according to manufacturers instructions. Briefly, $0.5 \mathrm{~mL}$ of $\sim 1 \mathrm{mg} / \mathrm{mL}$ Mic10 dissolved in $0.1 \%$ DDM was supplemented by $50 \mu \mathrm{L}$ of $1 \mathrm{M}$ sodium bicarbonate, $\mathrm{pH}$ 9.0. The mixture was transfered to the reactive dye (Alexa Flour 488) containing vial and stirred for $1 \mathrm{~h}$ at room temperature. Labeled Mic10 was separated from unbound dye by applying the solution to the pre-packed PD MidiTrap G-25 gravity columns (GE Healthcare). The separation procedure was carried out according to manufacturers instructions.

Site-directed labeling of recombinantly expressed Mic10 ${ }^{\mathrm{A} 5 \mathrm{C}}$, Mic10 A5C/G50/52A and Mic10A5C/G72/74A was achieved by employing Alexa Fluor 488 and Alexa Flour 568 maleimide fluorophores (Life Technologies). The labeling procedure was carried out according to manufacturers instructions. The protein was incubated with 25 molar excess dye. The samples were analyzed by FRET measurements (see 3.2.4.7).

\subsubsection{Protein membrane topology mapping}

Mic10 membrane topology mapping by site-directed cystein labeling was performed as described previously (Voeltz et al., 2006). Briefly, various yeast strains were generated (see Table 3.8 which expressed Mic10 ${ }^{\mathrm{A} 5 \mathrm{C}}$, Mic10 ${ }^{\mathrm{R} 62 \mathrm{C}}$ and Mic10 ${ }^{\mathrm{V} 97 \mathrm{C}}$. Mitochondria were isolated from these strains as described in 3.2.1.6. $40 \mu \mathrm{g}$ mitochondria were converted into mitoplasts by washing with $400 \mu \mathrm{L} 20 \mathrm{mM}$ Tris, $\mathrm{pH} 7.4$ buffer (osmotic swelling). The samples were gently mixed by twenty times pipetting and were incubated on ice for $25 \mathrm{~min}$. At this point the samples were split into two. The first $20 \mu \mathrm{g}$ samples were treated by 3-9 $\mu \mathrm{g}$ proteinase $\mathrm{K}$ (Roche) and incubated on ice for 10 min followed by addition of $1.5 \mu \mathrm{L} 0.2 \mathrm{M}$ PMSF and further $10 \mathrm{~min}$ incubation on ice. The samples were spun at $16000 \mathrm{xg}$ for $10 \mathrm{~min}$. The pellets were resuspended in sample loading buffer and analyzed by SDS-PAGE and immunoblot- 
ting.

The second half of the samples were used for labeling. The samples were incubated with $5 \mathrm{mM}$ maleimide PEG 2 KDa (Sigma Aldrich) with or without $2 \%$ SDS for $2 \mathrm{~h}$, shaking at $600 \mathrm{rpm}$. The reactions were terminated by incubating with $25 \mathrm{mM}$ DTT for $30 \mathrm{~min}$. The entire labeling procedure was carried out at $25^{\circ} \mathrm{C}$. The labeling success was monitored by SDS-PAGE and subsequent immunoblotting.

For Mic10 topology mapping in vitro, recombinantly expressed Mic10 and it's cystein variants from 3.2.3.9 were reconstituted into liposomes ( see 3.2.4.2). The incorporated Mic10 variants were labeled as described above. The labeling success was monitored by SDS-PAGE and subsequent coomassie staining.

\subsubsection{Mic10 co-immunoprecipitation}

For Mic10 co-immunoprecipitation, mitochondria were solubilized in digitonin buffer (20 mM Tris, $100 \mathrm{mM} \mathrm{NaCl,} 0.5 \mathrm{mM}$ EDTA, $10 \%$ glycerol, $1 \%$ digitonin, $\mathrm{pH}$ 7.4). Insoluble material was pellet by centrifugation for $10 \mathrm{~min}$ at $12000 \mathrm{xg}$ at $4{ }^{\circ} \mathrm{C}$. The soluble supernatant was loaded on ProteinA sepharose coupled with a $\alpha$ - Mic10 serum and incubated for $2 \mathrm{~h}$ at $4{ }^{\circ} \mathrm{C}$ under rotation. Afterwards the beads were washed in digitonin buffer containing $0.3 \%$ digitonin and the proteins were eluted with $100 \mathrm{mM}$ glycine $\mathrm{pH}$ 2.8. The eluted sample was analyzed by SDS-PAGE and subsequent immunoblotting.

\subsubsection{Handling of artificial lipid membranes}

\subsubsection{Liposome preparation}

Stock solutions of L- $\alpha-\mathrm{PC}, \mathrm{L}-\alpha-\mathrm{PE}, \mathrm{L}-\alpha-\mathrm{PI}, \mathrm{L}-\alpha-\mathrm{PS}$, DOPE, DMPC, CL and rhodamine PE were prepared in chloroform (Avanti Polar Lipids). For EM, FRET and fluorescence microscopy (GUVs) analysis LUVs were prepared by mixing either $40 \%$ L- $\alpha$-PC, $20 \%$ L- $\alpha$-PE, $5 \%$ L- $\alpha$-PS, $15 \%$ L- $\alpha$-PI, $15 \%$ CL and $80 \%$ L$\alpha-\mathrm{PC}$ to final concentration of $5-10 \mathrm{mg} / \mathrm{mL}$. In addition, large unilamellar vesicles (LUVs)/liposomes for fluorescence microscopy analysis contained $0.5 \%$ rhodamine PE. For NMR analysis liposomes were prepared from DMPC lipids to final concentration of $10 \mathrm{mg} / \mathrm{mL}$. Liposomes were prepared according to the standard procedure 
with slight modifications (Meinecke et al., 2006). Briefly, lipids dissolved in chloroform were mixed in indicated amounts in glass test tube. In order to obtain a thin lipid film and avoid lipid peroxidation, the lipid mixture was initially dried by flushing nitrogen gas, followed by $2 \mathrm{~h}$ incubation under vacuum in the desiccator. The lipid film was hydrated in appropriate amount of lipid hydration buffer $(100 \mathrm{mM} \mathrm{NaCl}$, $10 \mathrm{mM}$ Tris, $\mathrm{pH} \mathrm{8.0)}$ and vortexted for $30 \mathrm{~min}$. For obtaining the unilamellar vesicles, the mixture underwent twelve times freezing (in liquid nitrogen) and thawing (at room temperature) cycles with 2 min vortexing between the cycles. Subsequently, the mixture was filtered twenty one times through the $100 \mathrm{~nm}$ pore size polycarbonate membrane (Whatman). The size-distribution profile was analyzed by employing dynamic light scattering (DLS) (see 3.2.4.5).

\subsubsection{Proteo-liposome preparation}

Mic10 proteoliposome preparation was carried out as described before with slight modifications (van der Laan et al., 2007). Briefly, liposomes from 3.2.4.1 were solubilized in 0.05-0.1\% DDM for $15 \mathrm{~min}$ at room temperature by gentle shaking. Purified Mic10 renatured in $0.1 \%$ DDM (see 3.2.3.6) was added to different lipid to protein ratios to the detergent-containing liposome solution and incubated for $45 \mathrm{~min}$ on the shaker at room temperature. The actual proteoliposome formation was achieved by adding detergent adsorbent Bio-Beads SM-2 (Roth) with the amounts 1 gr beads per $240 \mathrm{mg}$ detergent to the mixture. The beads were incubated either for $2 \mathrm{~h}$ at room temperature or at $4^{\circ} \mathrm{C}$ for $16 \mathrm{~h}$.

Tim23-containing liposomes were prepared according to previously described protocol (Hinnah et al., 1997; Hill et al., 1998). Briefly, Tim23 dissolved in $8 \mathrm{M}$ urea from 3.2.3.6 was solubilized in $20 \mathrm{mM}$ Mega 9 for $15 \mathrm{~min}$ at room temperature by gentle shaking. Liposomes from 3.2.4.1 and Mega 9 with final $80 \mathrm{mM}$ concentration were added to the mixture. The mixture was further incubated for $45 \mathrm{~min}$ at room temperature on the shaker. The solution was ultimately transfered to the $3.5 \mathrm{KDa}$ pore size dialysis membrane (Spectrum Laboratories, Inc.) and dialyzed against $5 \mathrm{~L}$ buffer (100 mM NaCl, $20 \mathrm{mM}$ Tris, $\mathrm{pH} \mathrm{8.0)}$ ) for $2 \mathrm{~h}$ at room temperature and followed by $16 \mathrm{~h}$ dialysis at $4{ }^{\circ} \mathrm{C}$.

The effects of the proteins on liposome size were analyzed by DLS as discribed in 3.2.4.5. 


\subsubsection{Flotation assay}

For density flotation assay, nonionic histodenz was employed. Briefly, 40, 20, 10, 5 and $2 \%$ density gradient layers were prepared in the liposome hydration buffer. 50 $\mu \mathrm{L}$ liposomes or proteoliposomes from 3.2.4.1 and 3.2.4.2, respectively were loaded below the $40 \%$ layer and the discrete density gradient was built with the $2 \%$ layer at the top. The amount of each gradient was $800 \mu \mathrm{L}$. The gradient was centrifuged at $150,000 \times \mathrm{g}$ for $45 \mathrm{~min}$ and the liposomes / proteoliposomes were collected at the interfaces of the layers. The samples were TCA precipitated (see 3.2.3.8) and subjected to SDS-PAGE analysis.

\subsubsection{Sodium carbonate extraction}

Sodium carbonate extraction of proteoliposomes was carried out as described previously (van der Laan et al., 2007). Briefly, the proteoliposomes collected at the interfaces from 3.2.4.3 were treated by $200 \mathrm{mM} \mathrm{Na}_{2} \mathrm{CO}_{3}$ with the volume ratio of 1:10 proteoliposome:sodium carbonate and incubated for $30 \mathrm{~min}$ on ice. The samples were centrifuged at $150,000 \times \mathrm{g}$ for $45 \mathrm{~min}$ and supernatants and pellets were seperated. The supernatants were TCA precipitated (described in see 3.2.3.8). UItimately, membrane and TCA pellets were resuspended in SDS sample buffer and subjected to SDS-PAGE analysis.

\subsubsection{DLS measuments}

Dynamic light scattering size-distribution measurements were carried out by using a Zetasizer Nano S. The obtained data were an average of at least three independent measurements. Each measurement consisted of 20 repetitions over 10 min recording time. The errors were calculated as the standard deviation from the averaged values.

\subsubsection{Preparation of GUVs}

GUVs were prepared as described by (Girard et al., 2004). Briefly, GUVs were generated by employing electroformation technique developed by Angelova (Angelova 
and Dimitrov, 1986). Fluorescently labeled protein (see 3.2.3.10) was incorporated into LUVs (containing rhodamine PE) as described in 3.2.4.1. The proteoliposomes were collected for $30 \mathrm{~min}$ at 120,000 $\mathrm{xg}$ and resuspended in low-salt buffer (5 mM $\mathrm{NaCl}, 10 \mathrm{mM} \mathrm{HEPES}, \mathrm{pH} 7.4$ ) to a final $0.5 \mathrm{mg} / \mathrm{mL}$ lipid concentration. Subsequently, the proteoliposomes were deposited on indium tin oxide (ITO)-coated glass slides in $1 \mu \mathrm{L}$ droplets and dried for $2 \mathrm{~h}$ in a desiccator under $\mathrm{KCl}$ solution saturated vapor pressure. The two ITO-coated slides were separated by a thin O-ring silicone spacer, which was filled with $600 \mu \mathrm{L}$ of a $300 \mathrm{mM}$ glucose solution. The vesicle formation (electroformation) was achieved by generating AC (alternating current) electric field by a pulse generator connected to the slides. The field was applied for $3 \mathrm{~h}$ and incremented gradually from $20 \mathrm{mV}$ to $1.1 \mathrm{~V}$ at $12 \mathrm{~Hz}$ frequency. Ultimately, the field frequency was lowered to $4 \mathrm{~Hz}$ at $2 \mathrm{~V}$ for $30 \mathrm{~min}$, which leads the detachment of the formed vesicles from the glass slides. For microscopic visualization the GUVs were diluted in lower molar glucose buffer. The buffer osmolarities were measured using an osmometer.

\subsubsection{FRET measurements}

Förster (fluorescence) resonance energy transfer (FRET) measurements were carried out by employing the F-7000 fluorometer (Hitachi). For control Mic10-Alexa-488 and co-incorporated Mic10-Alexa-568 ${ }^{\text {acceptor }}$ and Mic10-Alexa-488 ${ }^{\text {donor }}$ FRET-pair the excitation wavelength was set to $493 \mathrm{~nm}$. For control Mic10-Alexa-568 and coincorporated Mic10-Alexa-568 acceptor and Mic10-Alexa-488 ${ }^{\text {donor }}$ FRET-pair the excitation wavelength was set to $574 \mathrm{~nm}$. Emission spectra was recorded between 470 and $700 \mathrm{~nm}$ at $60 \mathrm{~nm} / \mathrm{min}$. Sample preparation procedure was introduced in section 3.2.3.10.

\subsubsection{Visualization techniques}

\subsubsection{EM microscopy of LUVs}

Liposomes and proteoliposomes from 3.2.4.1 and 3.2.4.2 were diluted to achieve the final concentration of $0.5-0.8 \mathrm{mg} / \mathrm{mL}$. $5 \mu \mathrm{L}$ samples were spotted on carbon-coated 
grids (Agar Scientific) and incubated for $1 \mathrm{~min}$ at room temperature for sample adsorption onto the film surface. The excess sample was blotted off and the grids were briefly placed onto the $50 \mu \mathrm{L} 5 \%$ uranyl acetate solution droplet. The stain was immediately blotted off and the negative staining was achieved by placing the gird onto the fresh $50 \mu \mathrm{L} 5 \%$ uranyl acetate solution droplet. After $30 \mathrm{sec}$ the excess dye was blotted off.

For Mic10 immunogold staining (Wrede et al., 2013), after adsorption of Mic10containing liposomes on to the carbon-coated surface, the gird was placed onto a $50 \mu \mathrm{L} \alpha$-His antibody containing solution (antibody dilution 1:6000 in TBS) and incubated for $1 \mathrm{~h}$ at room temperature. Excess liquid was blotted off and the grid was washed two times for $2 \mathrm{~min}$ at room temperature on drops of $50 \mu \mathrm{L}$ TBS. Following the removal of an excess buffer the grid was incubated on the $50 \mu \mathrm{L}$ drop of a $10 \mathrm{~nm}$ gold particle conjugated to the secondary antibody for $45 \mathrm{~min}$ at room temperature (antibody dilution $1: 100$ in $0.5 \mathrm{M} \mathrm{NaCl}, 0.1 \% \mathrm{BSA}, \mathrm{pH} 7.4$ buffer). Subsequently, the grid was washed two times for 2 min on the drops of $50 \mu \mathrm{L}$ TBS and briefly rinsed on the water drop. The negative staining was achieved as described above.

To visualize the sample, the EM was employed. The electron microscopic imaging was performed with a Jeol JEM 1011 transmission electron microscopy with a Gatan Orius 1000 CCS camera.

\subsubsection{Electron microscopy of yeast cells}

Electron microscopy of yeast cells was performed by Dr. Daniel C. Jans at the Max Planck Institute for Biophysical Chemistry, Göttingen, Germany. Briefly, yeast cells grown at $30{ }^{\circ} \mathrm{C}$ in SGG $(0.05 \%$ yeast extract, $0.67 \%$ yeast nitrogen base, $0.2 \%$-HIS dropout mix (Sherman, 2002), $3 \%$ glycerol, $0.1 \%$ glucose) medium were collected in the logarithmic growth phase. The cells were fixed in $1.5 \% \mathrm{KMnO}_{4}$ (Erdmann et al., 1989). The cells were stained with $1 \%$ uranyl acetate for $2 \mathrm{~h}$ and subsequent to dehydration in a graded ethanol series, the cells were embedded in epoxide resin (Agar 100; Plano). The electron micrographs of ultrathin sections were taken by using a Philips CM 120 transmission electron microscope (FEI Europe). 


\section{Results}

\subsection{Mic10 induces high degrees of membrane curvature in model membranes}

\subsubsection{Purification of recombinantly expressed Mic10}

Knowledge about the possible role of the MICOS complex in inner mitochondrial membrane biogenesis has been essentially obtained by intense in organello biochemical studies in yeast (van der Laan et al., 2012). These studies provided valuable knowledge about the general impact of the MICOS complex on mitochondrial IM organization and to some extent cellular physiology. However, no information about the functional mechanisms of the complex or its single subunits has been reported. In order to address these problems, I started investigating the role of the Saccharomyces cerevisiae MICOS core subunit Mic10. For this I employed an in vitro bottom-up approach combined with the in organello studies. These studies require high amounts of sample. In many cases, proteins isolated from their native organisms cannot be obtained in sufficient quantities or within a reasonable timeframe.

In order to overcome these obstacles, I utilized recombinant protein expression in Escherichia coli. Mic10 was cloned into appropriate E. coli expression vectors and overexpression success and efficiency were monitored by SDS-PAGE analysis (Figure 4.1). Overexpression conditions were chosen to be $37^{\circ} \mathrm{C}, 1 \mathrm{mM}$ IPTG (Figure 4.1 lane 11 and Figure 4.3 lane 3). As Mic10 is an integral membrane protein, after overexpression in E. coli cells, it accumulated in high levels as insoluble inclusion bodies. The inclusion bodies were dissolved in either urea or guanidine hydrochloride $(\mathrm{GuHCl})$-containing buffer and Mic10 was isolated by using affinity tag $\left(\mathrm{His}_{6}\right)$ chromatography (Figure 4.3 lane 5).

In order to purify the protein to homogeneity, size-exclusion chromatography was 


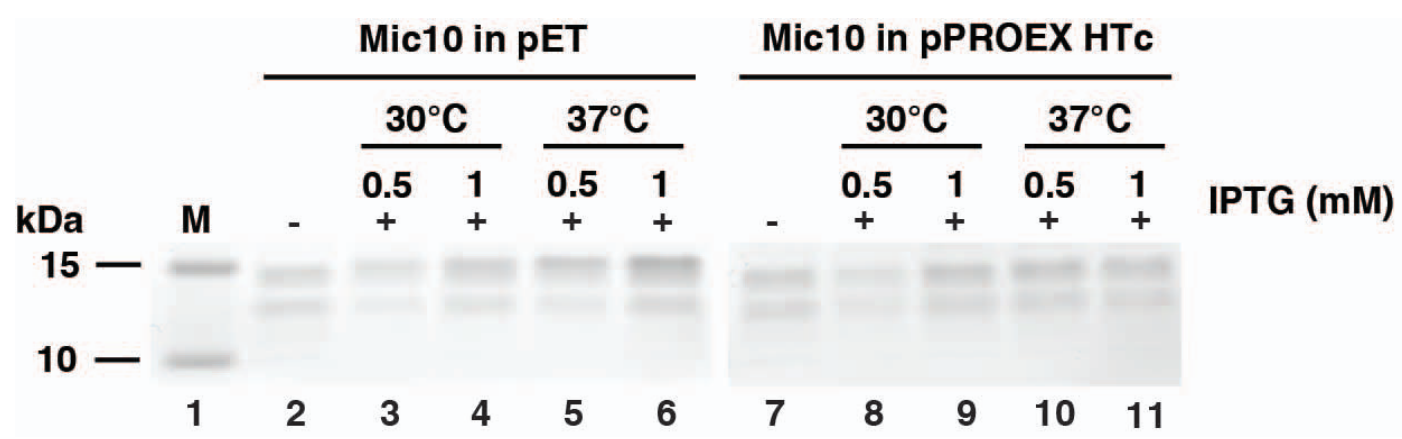

Figure 4.1: Mic10 overexpression test from different $E$. coli vectors. Following cloning of Mic10 gene into different E. coli vectors, Mic10 was overexpressed for $3 \mathrm{~h}$ at different IPTG and temperature conditions as indicated.

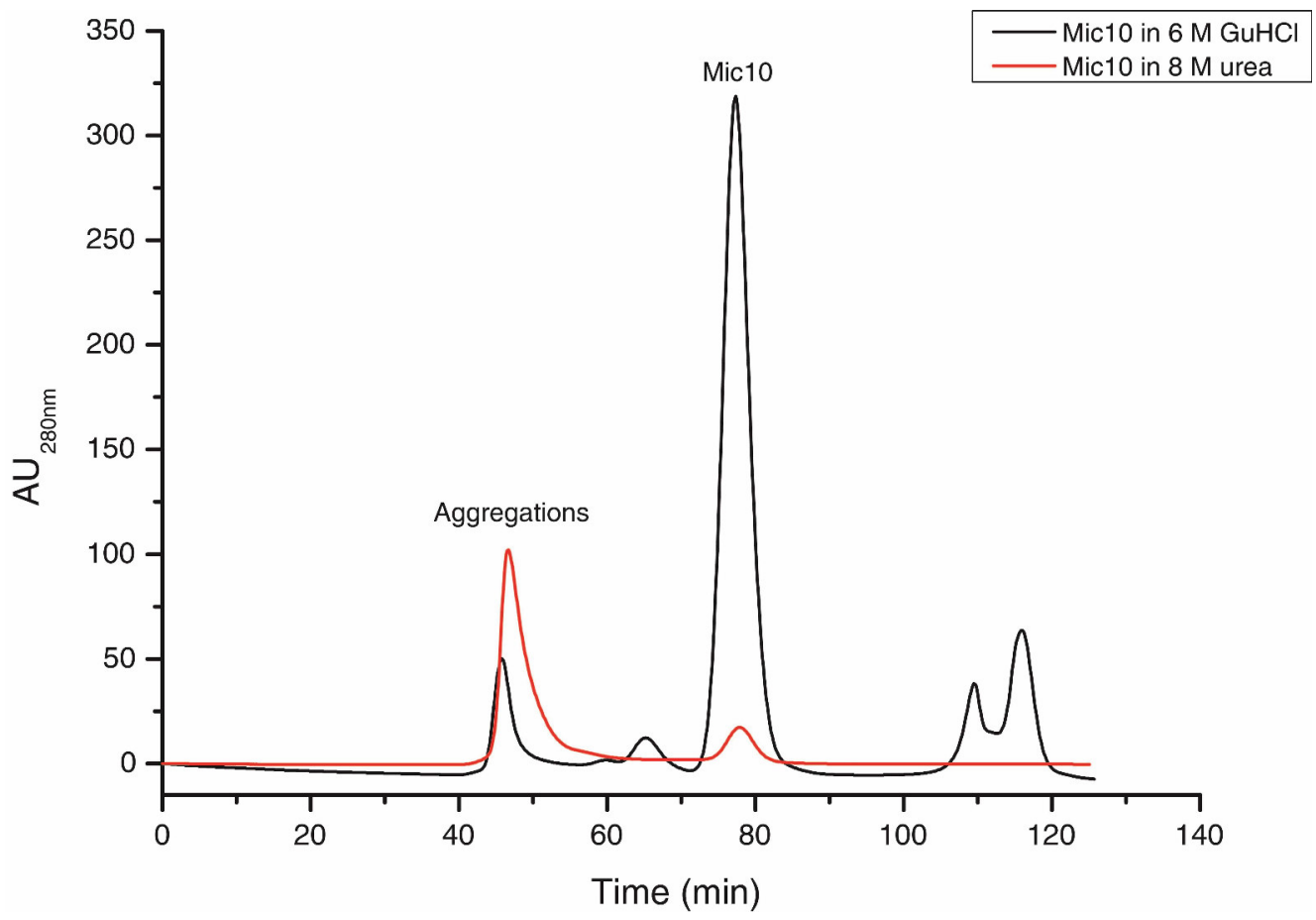

Figure 4.2: Mic10 aggregation recovery in chaotropic agents. Size-exclusion chromatography of previously purified Mic10 by affinity tag chromatography. Mic10 is dissolved in $8 \mathrm{M}$ urea (red line) and $6 \mathrm{M} \mathrm{GuHCl}$ (black line) containing buffer.

employed. Interestingly, when comparing the separation chromatograms of Mic10 dissolved in urea and $\mathrm{GuHCl}$ (loaded Mic10 amounts are not comparable), aggregation recovery of Mic10 dissolved in $\mathrm{GuHCl}$ was efficient. This was judged by observation of the lower aggregation peak height in comparison to the peak height 
corresponding to Mic10 molecules. In contrast, aggregation recovery of Mic10 in urea-containing buffer was inefficient (Figure 4.2). SDS-PAGE analysis confirmed the successful purification of Mic10 to homogeneity (Figure 4.3 lane 6).

$\mathrm{GuHCl}$ is a strong chaotropic reagent. Proteins and other macromolecules dissolved in buffers containing $\mathrm{GuHCl}$ in high molar concentrations, lose their ordered structure. In order to recover the native state of Mic10 and also maintain it in solution, $\mathrm{GuHCl}$ was dialyzed against buffer systems containing various non-denaturing, mild detergents. Following dialysis, Mic10-containing solutions were centrifuged, pellets and supernatants were separated and subjected to SDS-PAGE analysis. Mic10 was soluble at the indicated DDM and DM concentrations (Figure 4.4 lanes 5, 7, 9 and 11), but non-soluble at certain DDM concentration and in Mega 9 (lanes 3 and 13). DDM concentrations of $0.05 \%$ and $0.1 \%$ were chosen for the following experiments.

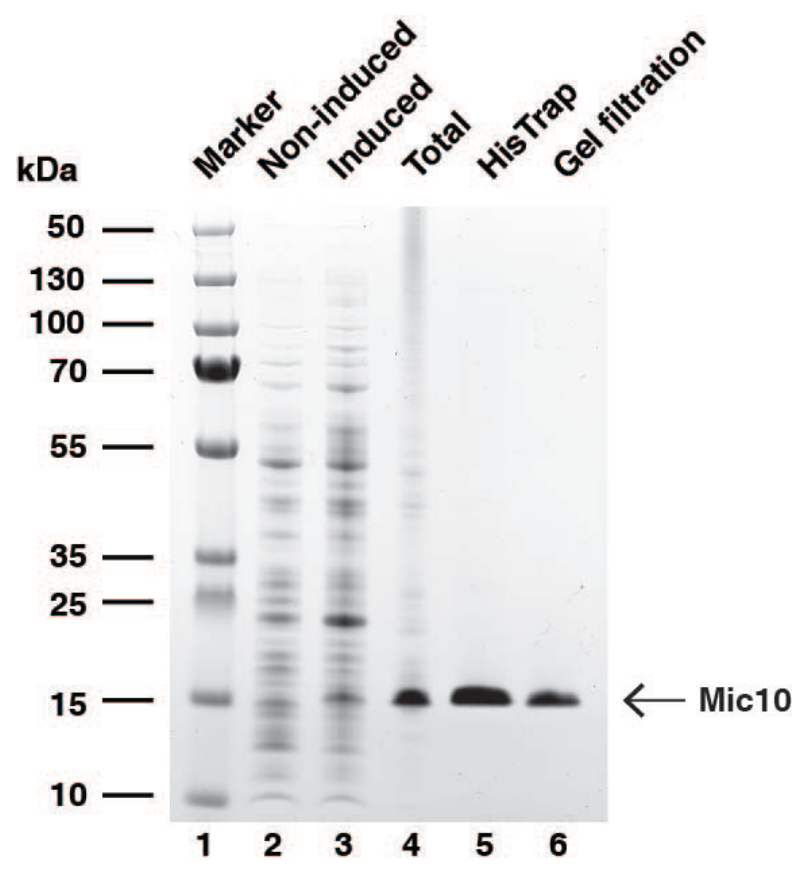

Figure 4.3: Expression and purification of recombinant Mic10. E. coli cells containing plasmid with the Mic10 gene were induced by addition of $1 \mathrm{mM}$ IPTG, at $37{ }^{\circ} \mathrm{C}$ for $3 \mathrm{~h}$. Lane 1 , molecular-weight size marker; lane 2, whole cell lysate of non-induced $E$. coli cells; lane 3 , whole cell lysate of induced E.coli cells, lane 4, purified inclusion bodies; lane 5, purified protein by affinity tag chromatography; lane 6 , purified protein by sizeexclusion chromatography. 


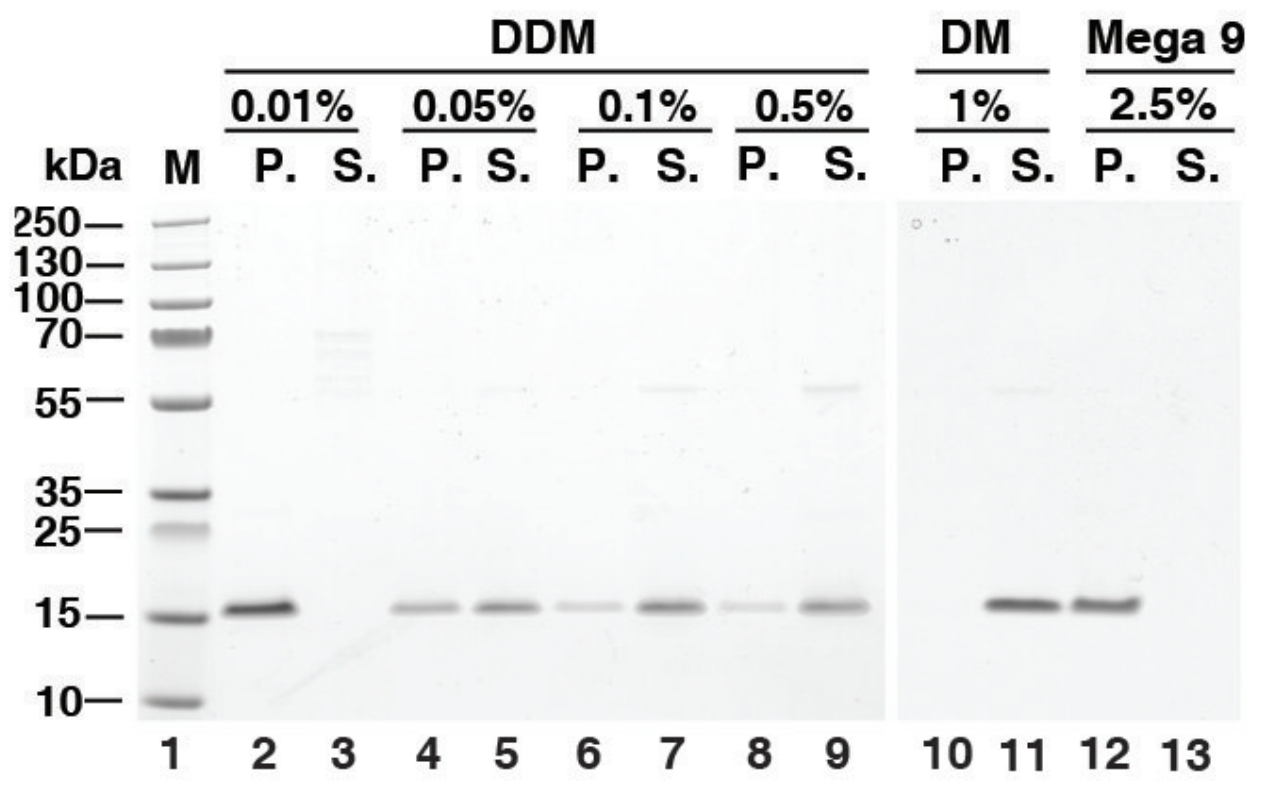

Figure 4.4: Solubility test of Mic10 in mild detergents. Mic10 dissolved in $\mathrm{GuHCl}$ containing buffer was dialyzed against buffers containing DDM, DM and Mega 9 at the indicated amounts. Following centrifugation the supernatants and pellets were analyzed by SDS-PAGE and subsequent Coomassie brilliant blue staining.

Thus, I established the optimal conditions for overexpression, purification and renaturing for $S$. cerevisiae Mic10 expressed in E. coli.

\subsubsection{Mic10 can be successfully integrated into model membranes}

In order to test whether Mic10 is able to induce any morphological changes in membranes, large unilamellar vesicles were employed as a model membrane system. LUVs mimicked inner mitochondrial membrane lipid composition and after extrusion had an average size of $120 \mathrm{~nm}$ diameter as confirmed by dynamic light scattering (DLS) measurements (Figure $4.7 \mathrm{~B}$ ). As Mic10 is an integral membrane protein, it was reconstituted into LUVs by using a detergent-mediated reconstitution protocol. Reconstitution efficiency was monitored by employing a non-ionic Histodenz discrete density gradient flotation assay with subsequent SDS-PAGE analysis (Figure $4.5 \mathrm{~A}$ (left), B). Mic10 incorporation into liposomes was successful, as proven by sedi- 
mentation of Mic10 by centrifugation after treating Mic10-containing liposomes with alkaline sodium carbonate solution (Figure 4.5 C).

A

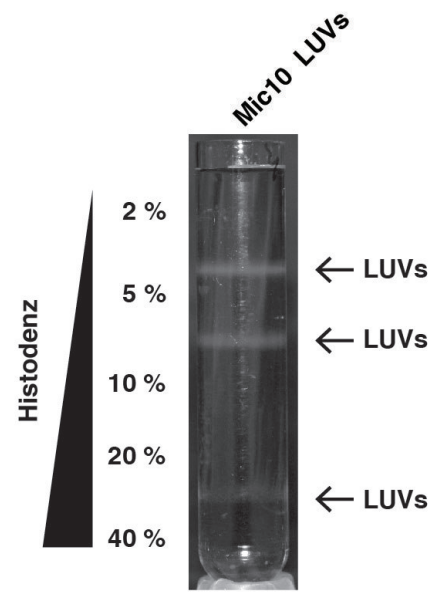

D
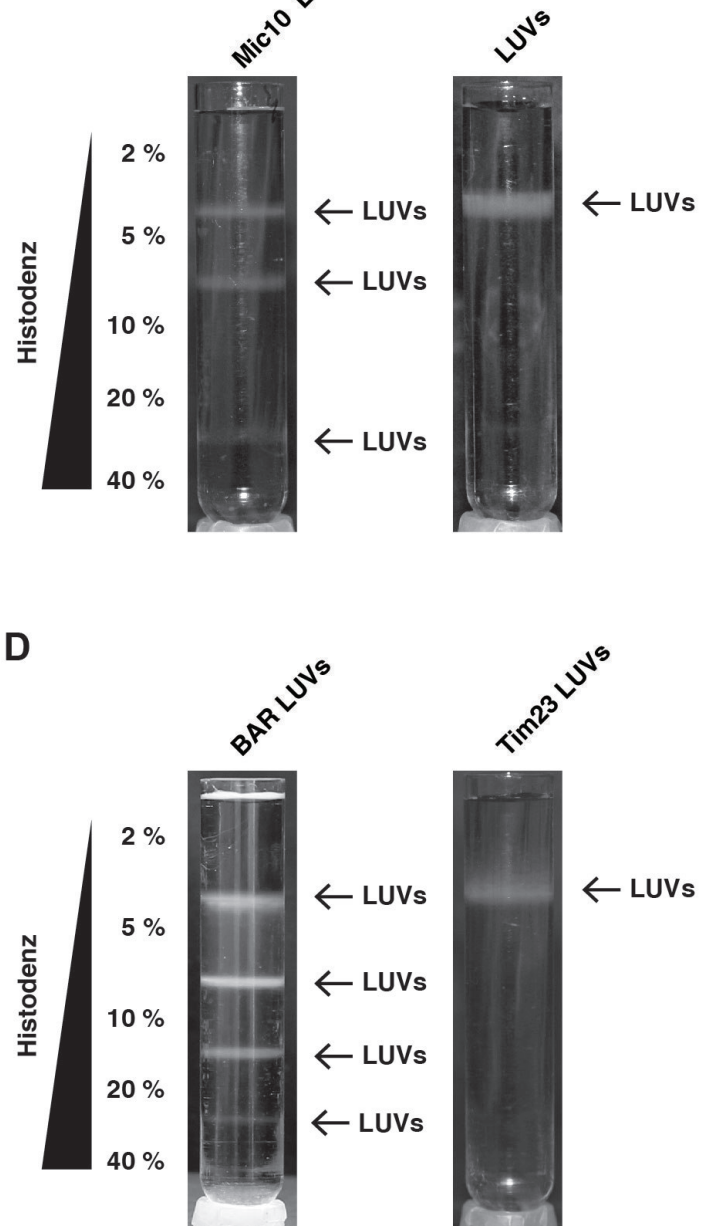

B

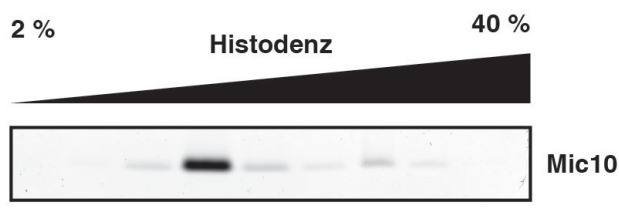

C

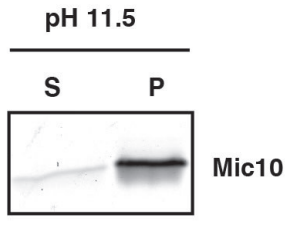

E

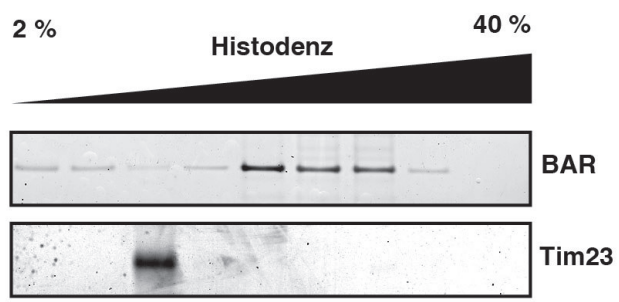

Figure 4.5: Recombinantly expressed Mic10 can be reconstituted into LUVs. A. Flotation assay of Mic10-incorporated vesicles (left) and mock incorporated vesicles (right) separated on a discrete density Histodenz gradient. B. SDS-PAGE analysis of fractionated gradient of Mic10-incorporated vesicles from $\mathbf{A}$ (left). C. SDS-PAGE analysis of sodium-carbonate treated Mic10-containing LUVs. D. Flotation assay of endophilin BAR-containing vesicles (left) and Tim23-incorporated vesicles (right) separated on a discrete density Histodenz gradient. E. SDS-PAGE analysis of fractionated gradients of endophilin BAR-containing vesicles (upper panel) from $\mathbf{D}$ (left) and Tim23-incorporated vesicles (lower panel) from D (right).

Interestingly, Mic10-containing vesicles were observed at various density lev- 
els in the gradient, unlike the empty LUVs, which underwent the same detergentmediated reconstitution procedure but without Mic10 (mock incorporation) (Figure 4.5 A, B). This observation suggests that Mic10 changes the overall structure of LUVs. Similar flotation patterns could be observed when LUVs were incubated with the BAR domain of endophilin (Figure 4.5D (left), E (upper panel)), a protein known to induce high degrees of membrane curvature (Farsad et al., 2001; Gallop et al., 2006; Meinecke et al., 2013). LUVs containing Tim23, a channel-forming protein of the inner mitochondrial membrane TIM23 translocase, showed flotation patterns similar to empty vesicles (Figure 4.5 D (right), E (lower panel)). Tim23 is predominantly localized in the inner boundary membrane and is not involved in membrane remodeling processes.

Thus the flotation assay of proteoliposomes, in addition to Mic10's successful incorporation into vesicles, gave us insight into this protein's possible influence on membrane morphology. However, we cannot draw more precise conclusions from this assay.

\subsubsection{Mic10 changes the morphology of model membranes}

In order to directly visualize the effects of Mic10 on liposome morphology, Mic10containing liposomes were analyzed by electron microscopy. Such liposomes showed broad structural changes in their morphology. Tubular membrane structures were repeatedly observed with diameters between 10 and $30 \mathrm{~nm}$ (Figure 4.6 A). Interestingly, vesicles and larger structures with internal tubular membrane structures were identified when the proteoliposomes were further incubated for $24 \mathrm{~h}$ at room temperature (Figure 4.6 A (middle and right)). No relevant structural changes of liposomes were observed with Mic10 concentrations below $200 \mathrm{nM}$, indicating the effect is protein concentration-dependent. Empty vesicles and Tim23-containing vesicles showed no significant alterations in membrane structures, whereas endophilin BARcontaining vesicles displayed characteristic tubular membrane shapes (Figure $4.6 \mathrm{~B}$, C, D, E) .

To address the effect of Mic10 on liposomes in bulk, the vesicle size distribution profile in response to different treatments was determined by DLS. As anticipated, only Mic10- and endophilin BAR-containing vesicles showed large-scale shifts in 
size distribution (Figures 4.7 A, E). Empty vesicles both, with and without mock incorporation and Tim23-containing vesicles displayed a homogeneous size distribution profile with an average $120 \mathrm{~nm}$ vesicle size (Figures 4.7 B, C, D). Interestingly, floating Mic10-containing liposomes from the bottom layers of the gradient showed more pronounced size distribution shifts than those from the upper layers (Figure 4.8).
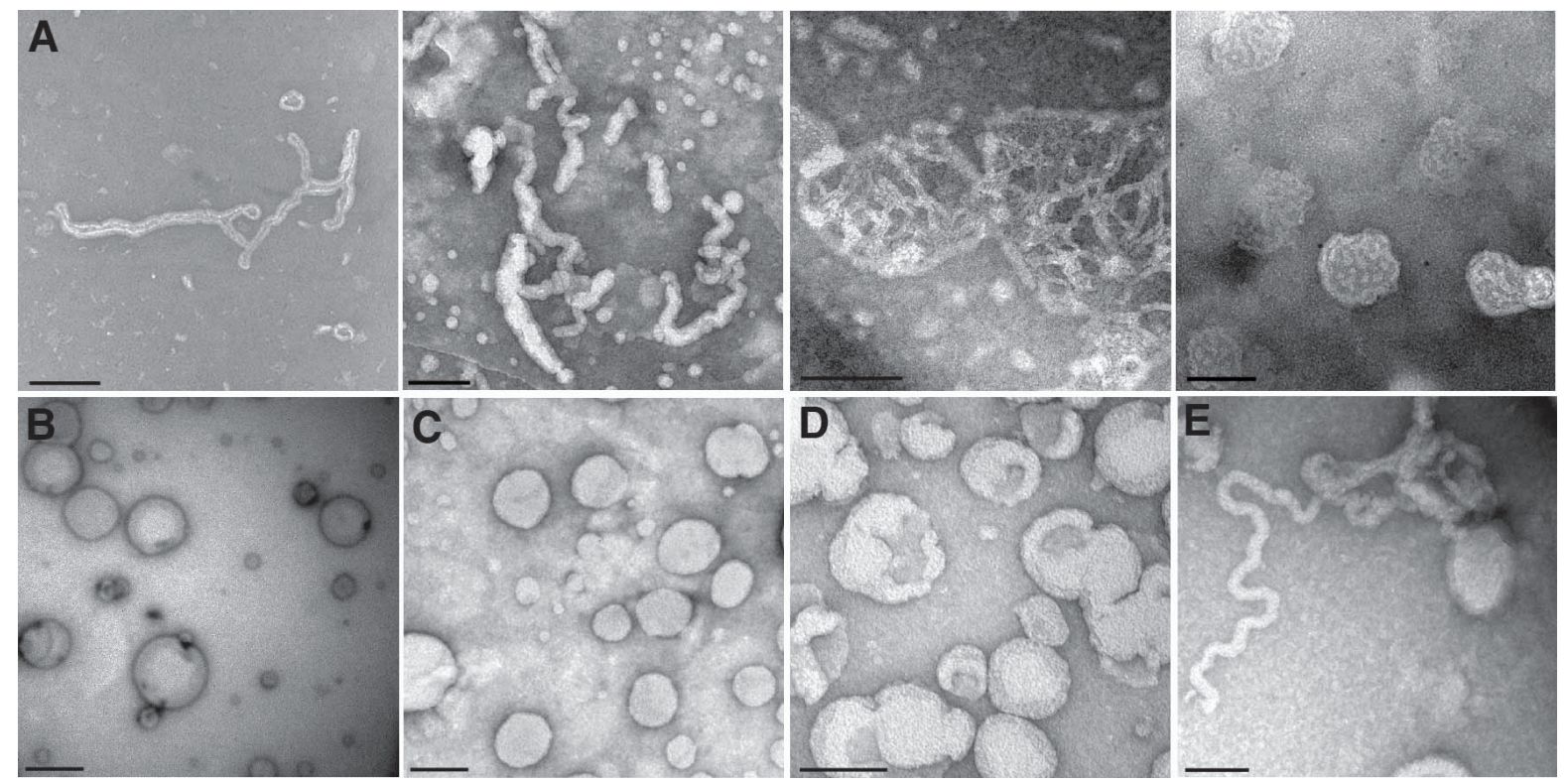

Figure 4.6: Mic10 changes morphology of LUVs. Electron micrographs of negatively stained A. Mic10-containing liposomes analyzed either directly (left panels) and after $24 \mathrm{~h}$ (right panels) incubation, B. LUVs without protein, C. LUVs that underwent the detergent-mediated reconstitution protocol in the absence of Mic10, D. Tim23-containing LUVs, E. LUVs incubated with endophilin BAR. Scale bars, $100 \mathrm{~nm}$. Electron micrograph $\mathbf{E}$ was taken by Benjamin Kroppen from our group. 
A

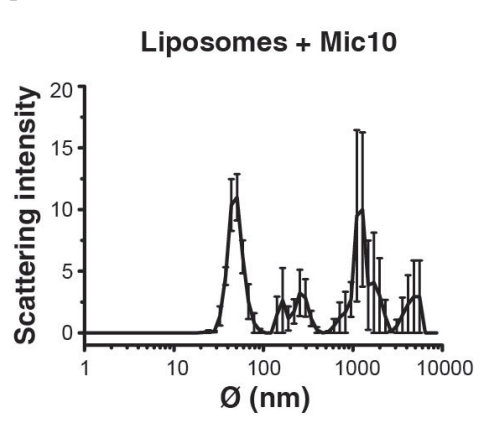

D

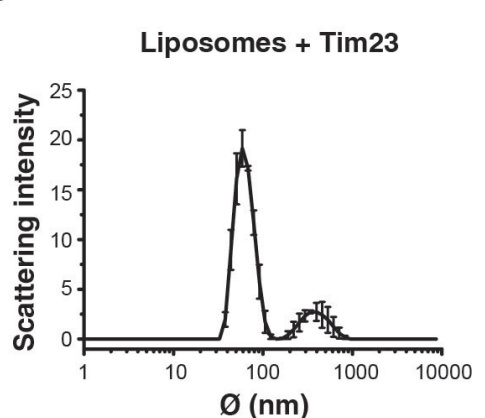

B

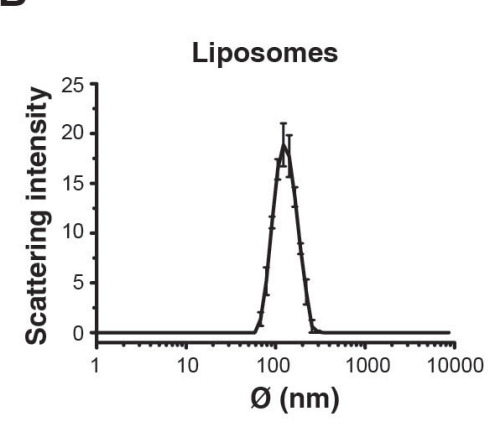

E

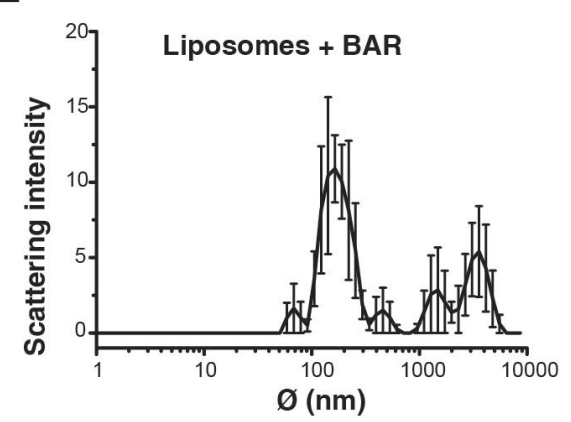

C

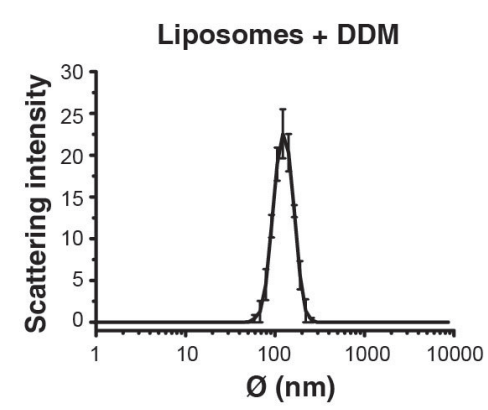

$A$

D্

$\stackrel{\infty}{\text { क }}$

Figure 4.7: Mic10 changes the size of liposomes in bulk. Average size distribution profile of A. Mic10-containing LUVs, B. LUVs without protein, C. LUVs, which underwent the detergent-mediated reconstitution protocol in the absence of protein, D. Tim23-containing LUVs and E. LUVs incubated with endophilin BAR measured by DLS. 
A

$40-20 \%$ Histodenz

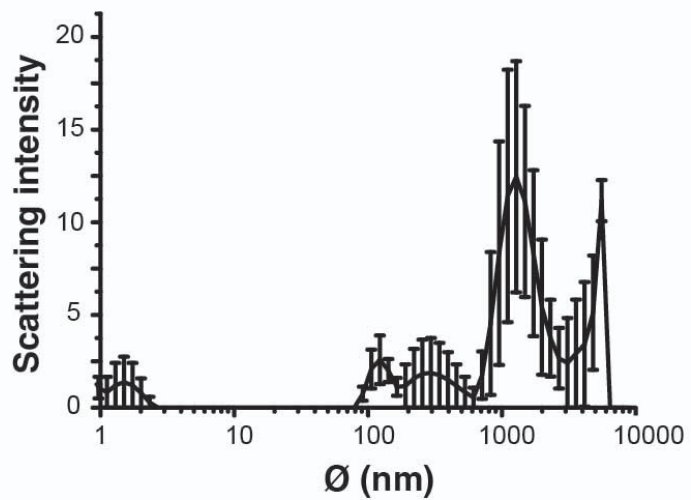

B

$5-2 \%$ Histodenz

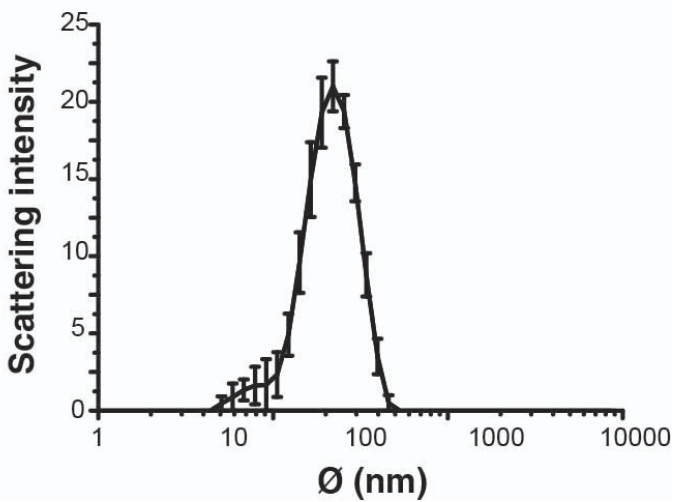

Figure 4.8: Size distribution profile of Mic10-containing liposomes after flotation . Average size distribution profile of Mic10-containing LUVs after flotation from the A. bottom and B. upper layers with indicated Histodenz densities, measured by DLS.

In order to examine whether liposome morphology changes can be attributed to membrane-integrated Mic10, the effects of Mic10 were studied using giant unilamellar vesicles. GUVs are another model membrane system whose physico-chemical properties differ from those of LUVs. To this end, fluorescently labeled Mic10 was incorporated into LUVs containing the fluorescently labeled lipid rhodamine PE and GUVs were produced from these proteoliposomes by the electroformation. Incorporation of Mic10 into GUVs caused drastic membrane morphology changes with a massive accumulation of internal tubular and vesicular structures as visualized by confocal fluorescence microscopy (Figure 4.9 A (lower panel), B (middle panel)). In contrast, Tim23 led to no apparent morphological changes in GUVs. Notably, these internal membrane structures were evenly decorated with Mic10. A similar pattern was observed when the immuno-gold-labeled Mic10 induced tubular structures were examined with electron microscopy (Figure 4.10).

In conclusion, S.cerevisiae Mic10 was isolated from E.coli in a functionally competent state and can induce high degrees of membrane curvature in model membrane systems with different physico-chemical properties. 
A

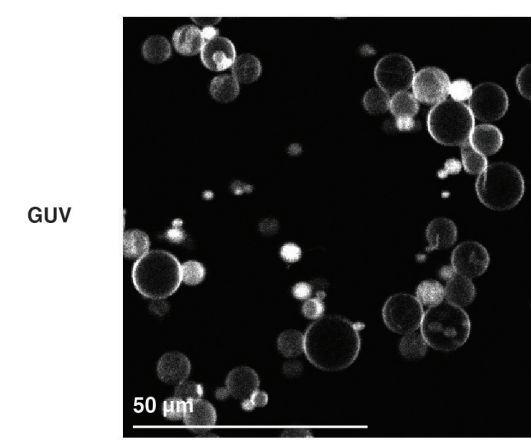

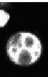

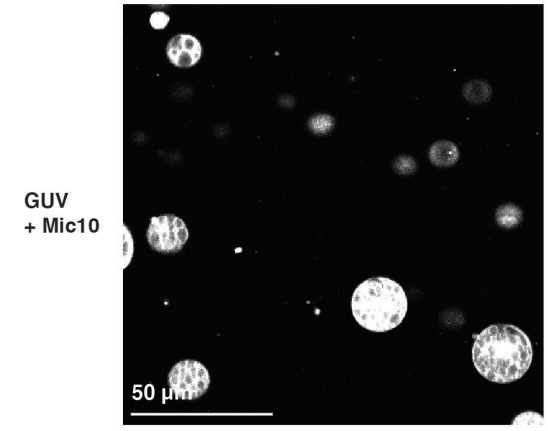

B
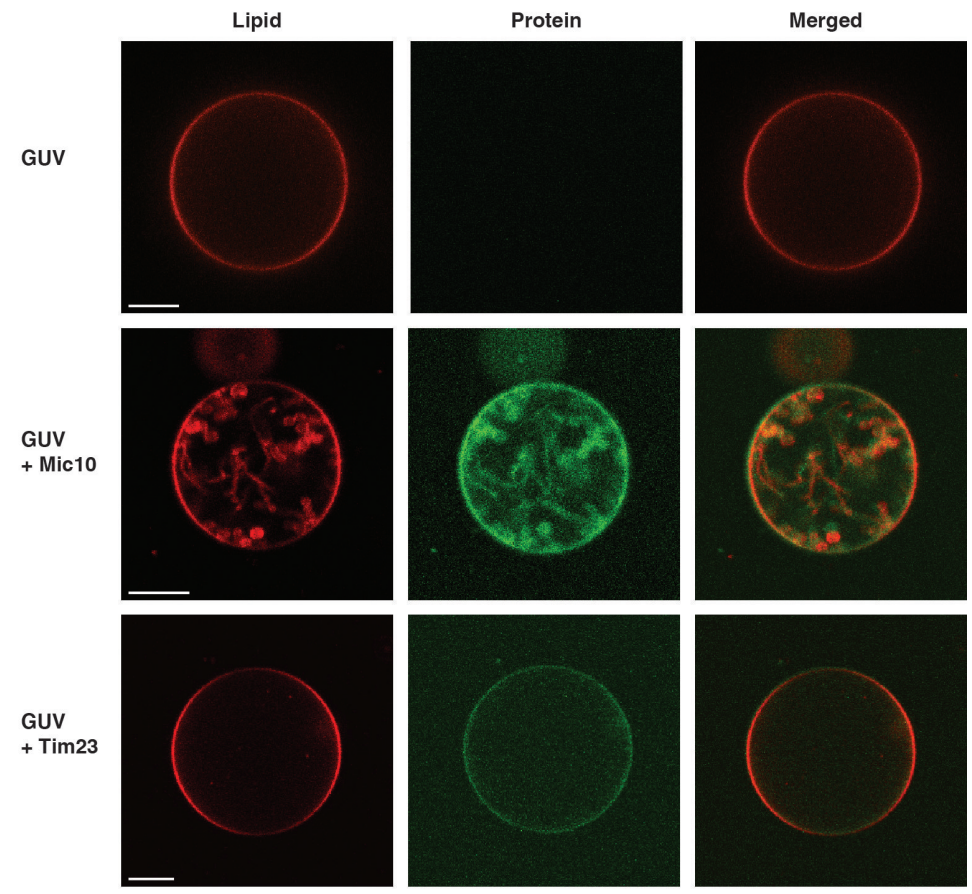

C

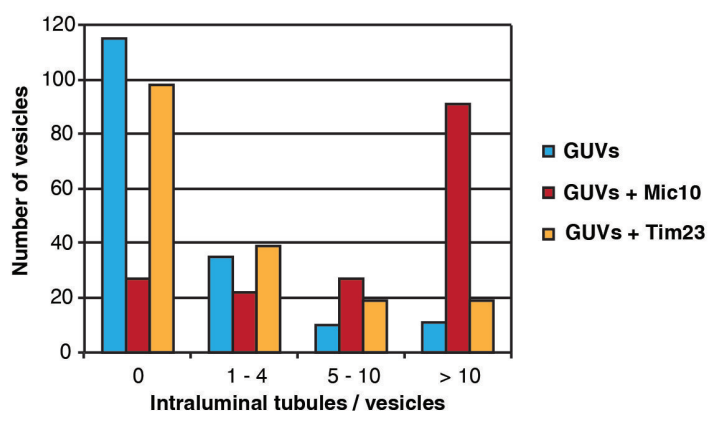

Figure 4.9: Mic10 changes morphology of GUVs. Confocal fluorescence microscopy images of $\mathbf{A}$. GUVs (labeled with rhodamine-PE) in the absence (upper panel) and in the presence of Alexa-488 labeled Mic10 (lower panel). Scale bars, $50 \mu \mathrm{m}$. B. upper and middle panels as in $\mathbf{A}$ and GUVs in the presence of Tim23 (lower panel). Scale bars, $10 \mu \mathrm{m}$. 

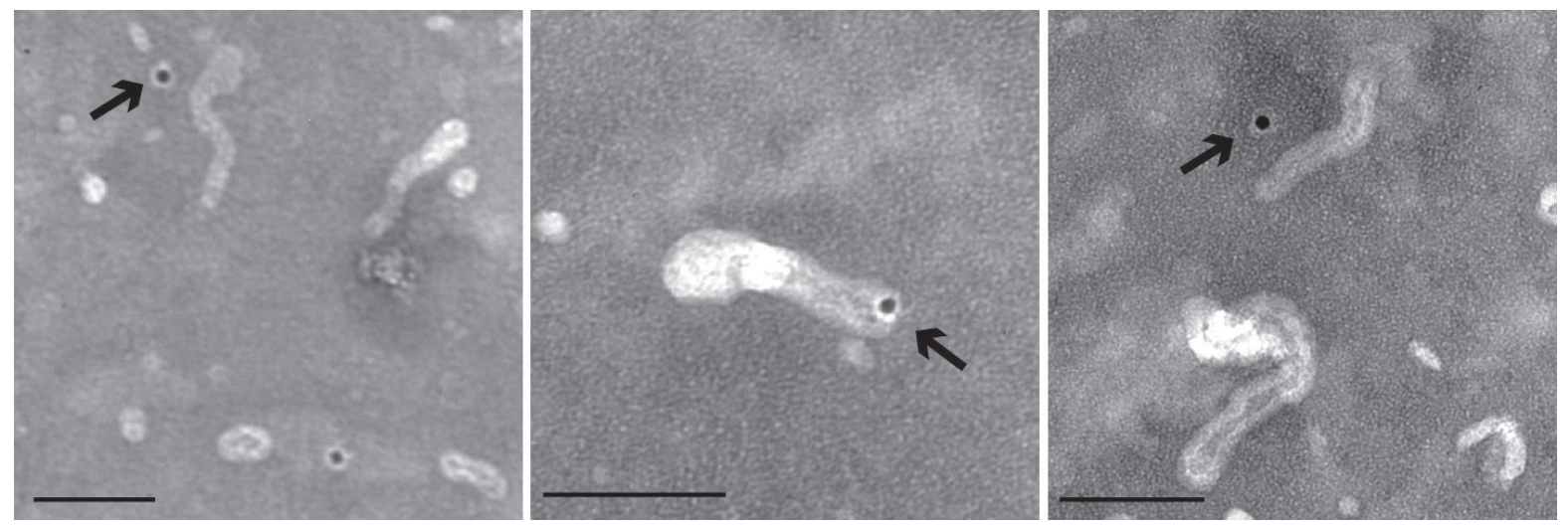

Figure 4.10: Mic10 is evenly distributed in liposomes. Electron microscopy images of tubular liposome structures containing immuno-gold labeled Mic10. Arrows indicate gold particles which could project a distance $(\sim 20 \mathrm{~nm})$ from the tubules due to the size of the linking antibodies.

\subsection{Mic10 membrane topology mapping}

\subsubsection{Trials to obtain Mic10 structure}

In order to understand the mechanism of membrane morphology changes by Mic10, we undertook EM analysis of detergent solubilized Mic10. For this purpose, Mic10 was dissolved in $0.1 \%$ DDM and analyzed by negative staining EM for different protein concentrations. Interestingly, at high protein levels (0.05 and $0.1 \mathrm{mg} / \mathrm{ml}) \mathrm{Mic} 10$ was mainly assembled into large structures, whereas at low amounts $(0.01 \mathrm{mg} / \mathrm{ml})$, the protein was found in its monomeric forms (Figure 4.11 middle and right panels and left panel, respectively). The obtained structures are rather heterogeneous. Hence, optimization of sample preparation is required.

In addition, we performed the solid state NMR spectroscopy trials of Mic10 incorporated into liposomes. Mic10 was expressed in M9 minimal media (see 3.2.1.1) supplemented with ${ }^{13} \mathrm{C}$ and ${ }^{15} \mathrm{~N}$ under different conditions (Figure $4.12 \mathrm{~A}$ ). The protein was purified to homogeneity and solubilized in DDM, as described in section 4.1.1. Mic10 was successfully incorporated into liposomes at different lipid-to-protein ratios as judged by flotation and sodium-carbonate assays (Figure $4.12 \mathrm{~B}$ and $\mathrm{C}$ ). The sample was subjected to solid state NMR measurements, however the procedure remains problematic due to issues such as a low signal-to-noise ratio and 
protein dilution effects due to lipid presence. Thus, the optimization of solid state NMR conditions is still ongoing.
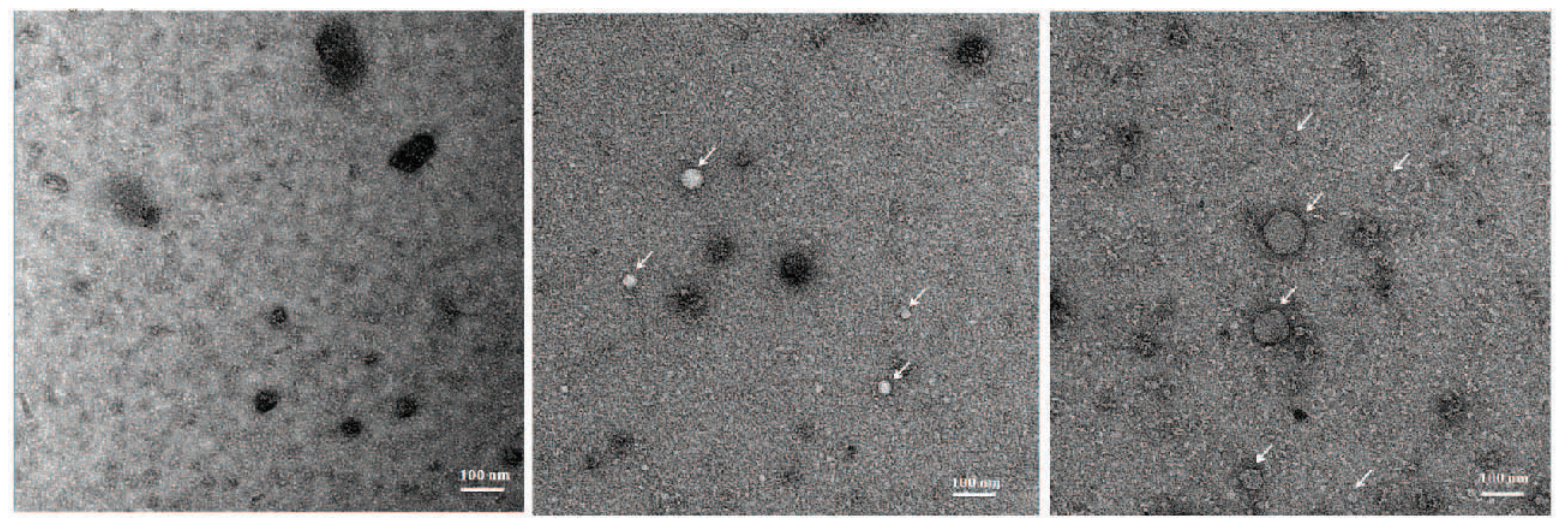

Figure 4.11: EM micrographs of Mic10 dissolved in detergent. Mic10 dissolved in $0.1 \%$ DDM was analyzed by negative staining EM. The figures depict the Mic10 concentration-dependent assembly from monomers (white dots) to larger structures (white circles). The EM micrographs were taken by Dr. Naoko Mizuno, Max Planck Institute of Biochemistry, Martinsried, Germany.

A

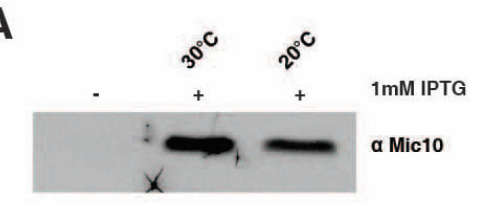

C

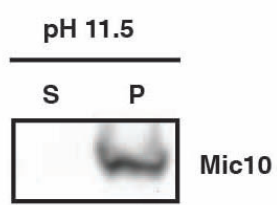

B $2 \%$ Histodenz $40 \%$

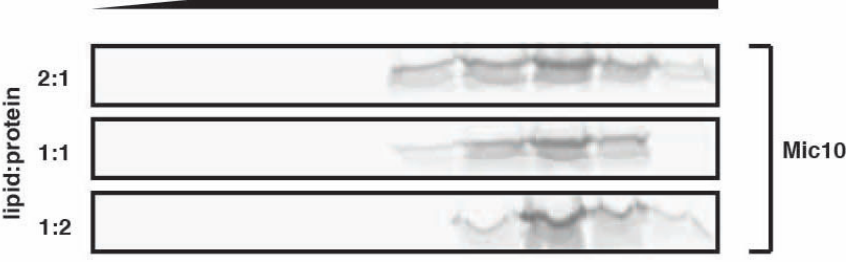

Figure 4.12: Sample preparation for solid state NMR. A. Overexpression test of Mic10 in M9 minimal media at indicated experimental conditions, analyzed by SDS-PAGE and subsequent immunoblotting, B. Flotation assay of Mic10-containing liposomes at indicated lipid-protein ratios analyzed by SDS-PAGE, C. SDS-PAGE analysis of carbonate-treated Mic10containing liposomes (lipid:protein 2:1) after flotation assay. 


\subsubsection{Mic10 spans the mitochondrial inner membrane in a hairpin topology}

As a next step, we set out to determine the topology of Mic10 in the inner mitochondrial membrane. It has previously been shown that the C-teminus of Mic10 is exposed to the IMS. Based on this knowledge and controversial opinions about the number of Mic10 transmembrane segments, two different Mic10 membrane topology models have been suggested (Harner et al., 2011; von der Malsburg et al., 2011; Alkhaja et al., 2012).

In order to experimentally map the topology of Mic10, site-specific maleimidePEG ( $2 \mathrm{kDa}$ ) covalent labeling of Mic10 at introduced cysteins was used. The cystein positions were chosen such a way as to distinguish between two different probable topologies (Figure 4.13). To this end, various yeast constructs were generated that expressed Mic10 cystein mutants Mic $10^{\mathrm{A} 5 \mathrm{C}}$, Mic $10^{\mathrm{V} 97 \mathrm{C}}$ and Mic $10^{\mathrm{R} 62 \mathrm{C}}$ corresponding to the $\mathrm{N}$ - and $\mathrm{C}$-terminus and the midpoint of predicted two transmembrane segments, respectively. Mitochondria were isolated from these strains and mitoplasts

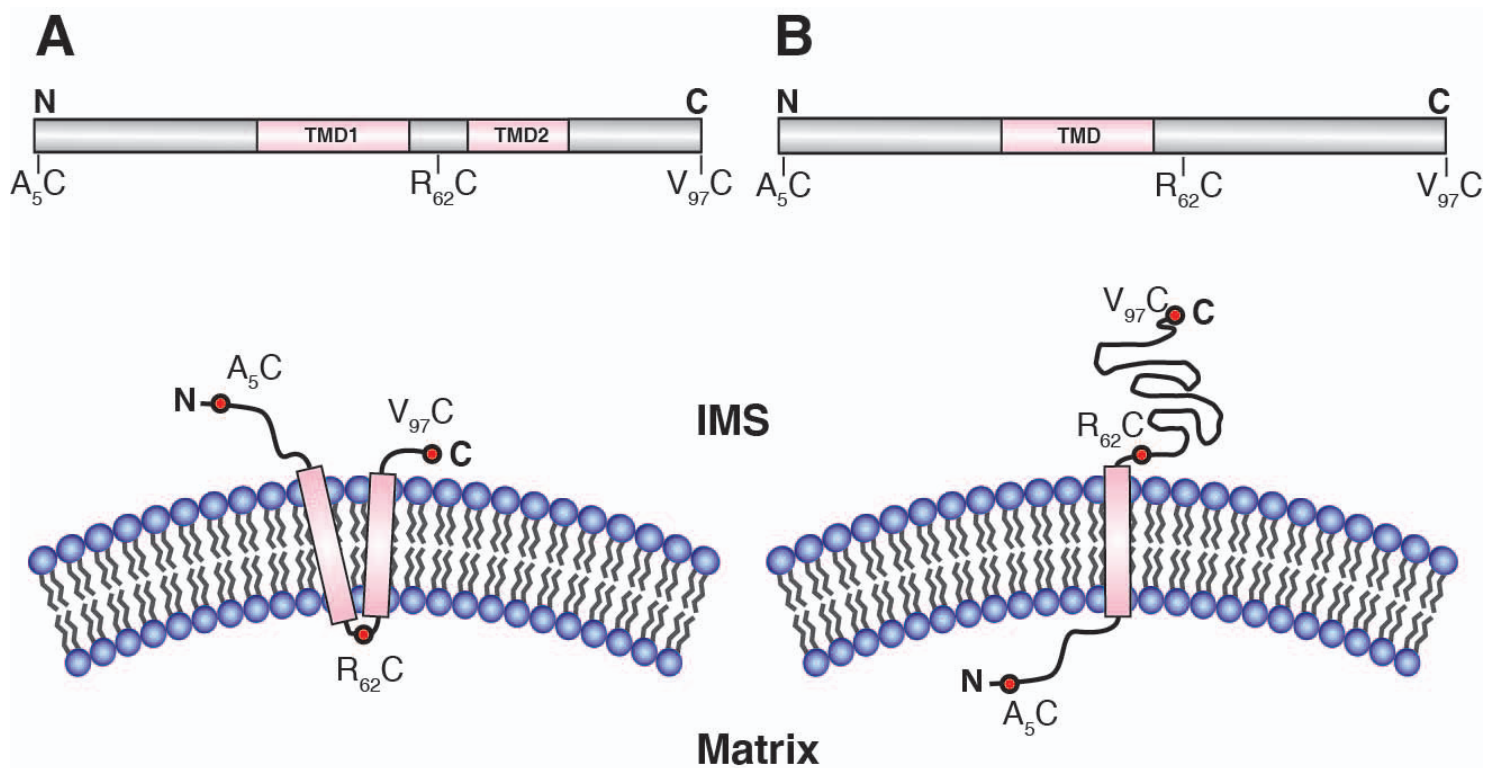

Figure 4.13: Cartoon of two putative topologies of Mic10 in the inner mitochondrial membrane. A. Hairpin topology of Mic10 within the inner mitochondrial membrane based on the presence of two transmembrane domains. B. Topology of Mic10 in the presence of single transmembrane domain.

were generated by resuspending mitochondria in a hypotonic buffer. The membraneimpermeable substrate maleimide-PEG $(2 \mathrm{kDa})$ was added to the mitoplasts and its 
A

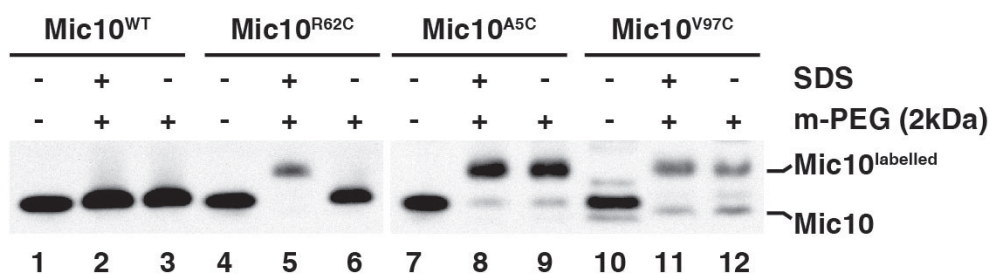

B

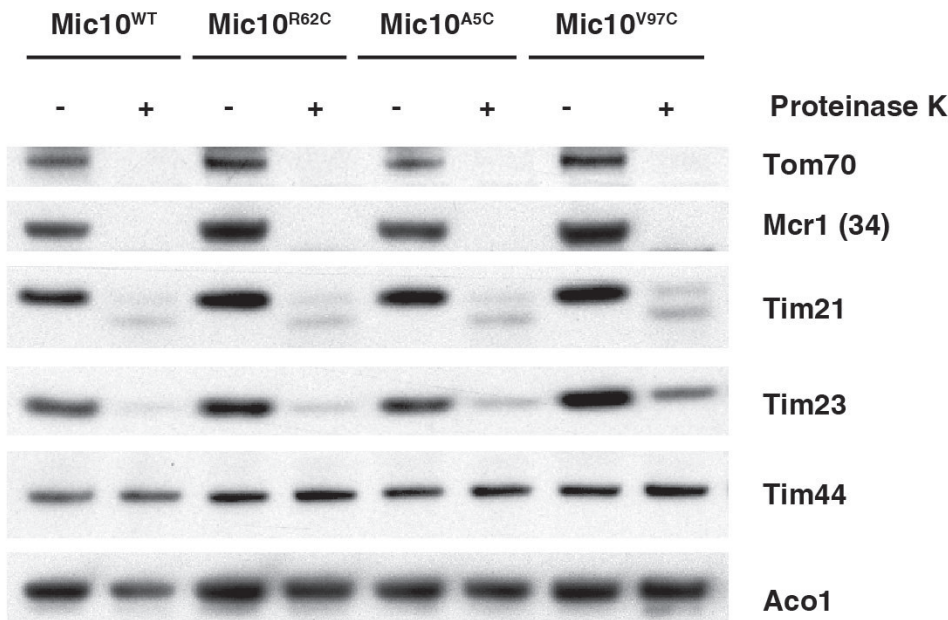

C

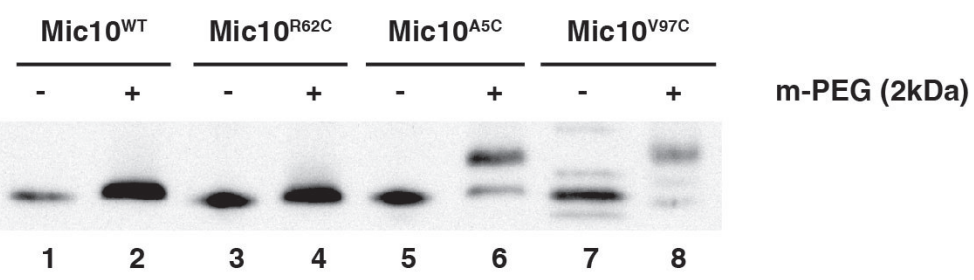

Figure 4.14: Topology mapping of Mic10 in inner mitochondrial membrane. A. Following disruption of the outer membrane, the cysteins introduced in Mic10 at the indicated positions were labeled by adding maleimide PEG (2kDa) under the indicated conditions, B. Mitoplasts were treated by proteinase $\mathrm{K}$ and their integrity was monitored by immunodecorating of a number of indicated mitochondrial proteins, $\mathbf{C}$. Mitochondria containing indicated Mic10 variants in the presence and absence of maleimide PEG (2kDa).

accessibility to cysteins was judged by size-shifts on SDS-PAGE gels followed by immunoblotting analysis. Interestingly, Mic $10^{\mathrm{A} 5 \mathrm{C}}$ and Mic $10^{\mathrm{V} 97 \mathrm{C}}$ were efficiently labeled, 
whereas Mic10 ${ }^{\mathrm{R} 62 \mathrm{C}}$ was not (Figure 4.15 A (lanes 9, 12 and 6). This indicates that cysteins at the $\mathrm{N}$ - and $\mathrm{C}$-terminus were exposed towards the IMS and the cystein at position 62 points towards the matrix. Although the outer membrane of mitochondria was partially permeable to the maleimide PEG (Figure 4.15 C (lanes 6 and 8)), conversion of mitochondria into mitoplasts was efficient with the inner membrane retained intact. This was proven by the accessibility of the IMS-exposed inner membrane proteins Tim21 and Tim23 to proteinase $\mathrm{K}$ and PK-preserved matrix-exposed inner membrane proteins Tim44 and Aco1 (Figure 4.15B). Moreover, all constructs were efficiently labeled upon treating with SDS (Figure 4.15 A (lanes 5, 8 and 11). Thus, control experiments confirmed the validity of the data.

In conclusion, Mic10 membrane topology mapping experiments revealed that, Mic10 contains two transmembrane domains and spans the IM with a hairpin topology with $\mathrm{N}$ - and $\mathrm{C}$ - termini exposed to the IMS (Figure $4.13 \mathrm{~A}$ ).

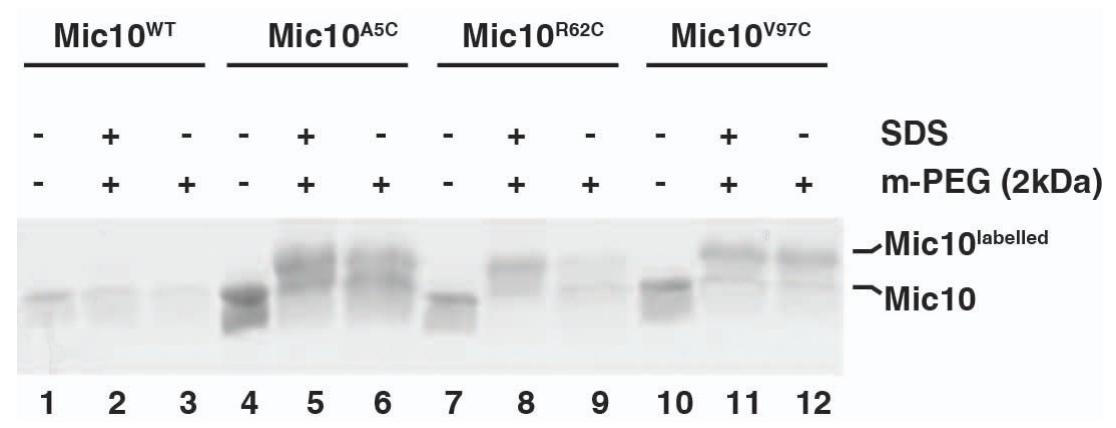

Figure 4.15: In vitro Mic10 membrane topology mapping. Indicated recombinantly expressed Mic10 cystein variants were purified to homogeneity and labeled by additon of maleimide-PEG (2kDa). The labeling success was monitored by SDS-PAGE and Coomassie brilliant blue staining.

In addition to in organello Mic10 membrane topology determination, we set out to determine the topology of recombinantly expressed Mic10 in liposomes. To this end, different cystein variants (as for native Mic10) Mic10 ${ }^{\mathrm{A} 5 \mathrm{C}}$, Mic10 ${ }^{\mathrm{V} 97 \mathrm{C}}$ and Mic10 ${ }^{\mathrm{R} 62 \mathrm{C}}$ were purified to homogeneity from E.coli cells (purification steps are not shown in this thesis) by applying the methods described in section 4.1.1. The Mic10 cystein variants were incorporated into LUVs independently from each other. Maleimide PEG (2kDa) was added to each proteoliposome solutions. As judged from the SDSPAGE, all Mic10 cystein variants were partially or inefficiently labeled, making it impossible to draw an unambiguous conclusion on Mic10's topology in model mem- 
branes.

Hence, optimization of sample preparation and labeling conditions will be necessary to determine the topology of Mic10 in model membranes.

\subsection{Mic10 forms homo-oligomers through glycine-rich motifs}

\subsubsection{Mic10 forms homo-oligomers}

It has been previously suggested that Mic10 might form homo-oligomers in the inner mitochondrial membrane (Harner et al., 2011). In addition, oligomerization or clustering of a number of membrane curvature inducing proteins have been shown in various studies (Paumard et al., 2002; Voeltz et al., 2006). Mic10 has two highly conserved glycine-rich motifs in both transmembrane domains (Figure 4.16A). Such glycine-rich motifs in transmembrane helices are shown to promote protein-protein interactions and ultimate protein oligomerization (Russ and Engelman, 2000).

In order to experimentally show if Mic10 forms oligomers, recombinantly expressed Mic10 solubilized in urea and DDM-containing buffer was subjected to SDSPAGE analysis. Interestingly, higher molecular-weight bands were detected in the lane corresponding to Mic10 dissolved in DDM (Figure 4.16 B, lane 2) and the number of bands increased considerably as detected after immunodecoration with an antibody specific for Mic10 (Figure 4.16 B, lane 3).

To validate that the bands detected on SDS-PAGE gels represent the Mic10 oligomers, Mic10 solutions in the mild detergents DDM and digitonin were subjected to BN-PAGE analysis. As judged from BN-PAGE gels, the majority of Mic10 was in an oligomeric form, confirming the protein's ability to oligomerize (Figure 4.16 C). 
A

Mic 10_H.sapiens

Mic 10_R.narvegus

Mic10_D.rerio

Mic10 S.cerevisiae

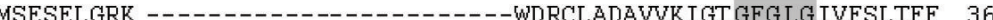

MSESEPGRK ---------------WDRCMADALVKLGT GFGLGMVFSLTFF 36

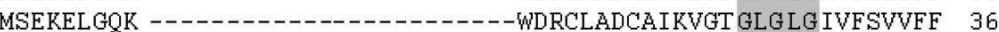

MSEOAOTOQPAKSTPSKDSNK HGSSVSTILDTKWD IVLSMML VKTAM GFGVGVFTSVLFF 60

Mic10_S. pombe MSTSQSSEQ --.---.--

Mic10_H.sapiens KRRMWPLAFGSGMGLGMAYSNCQHDFQAPYLLHGKYVKEQEQ--- 78

Mic10_R.norvegus KRRKWPLAFGSGVGLG MAYSNCQHDFQAPYLLHGKYVKEQ----- 76

Mic10 D.rerio KRRTSPIALGTGIGLG MAYSNCONDLRSHYOLHRSAAKEQ----- 76

Mic10_S.cerevisiae KRR BFPVWL GI GFGVG RGYAEGDAIIFRS----SAGLRSSKV---- 97

Mic10_S.pombe RRAAWPVWGGL GFGLG KSYADSNARLRTFHAIPKQLPASSTQKKD 86

$* * * * * * * * *: *:$

B

C

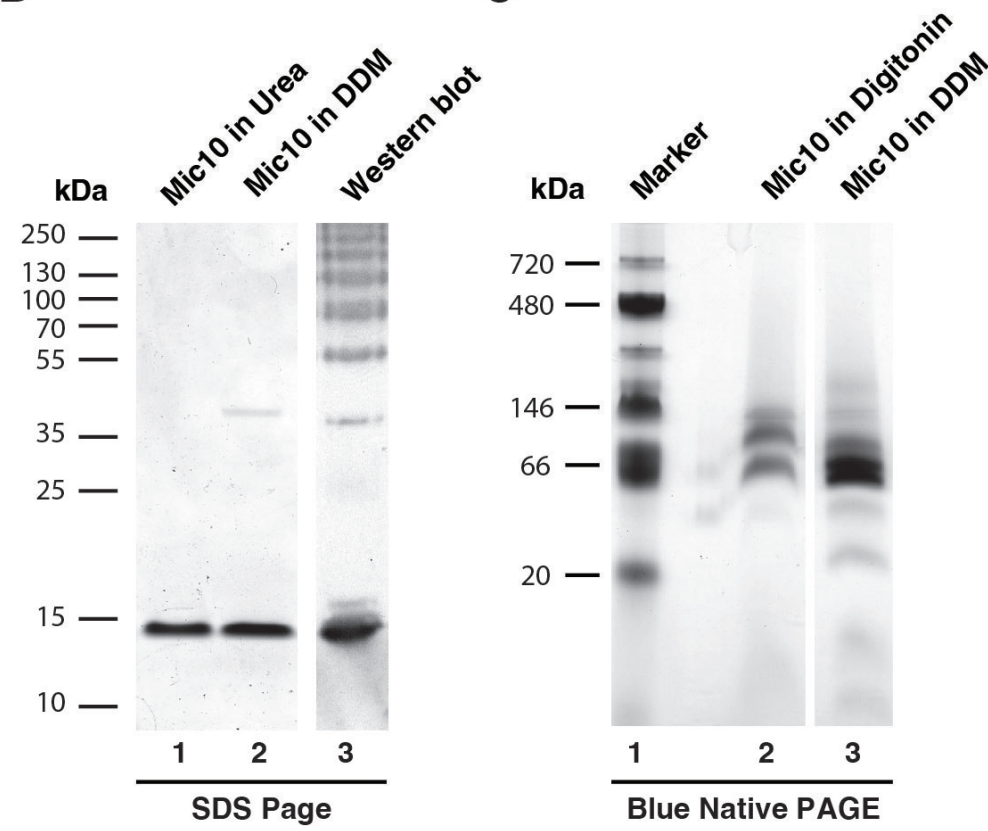

Figure 4.16: Mic10 forms homo oligomers. A. Sequence alignment (ClustalW) of Mic10 from different species. Red boxes indicate predicted transmembrane domains. Grey boxes mark conserved GxxxG motifs, B. SDSPAGE and immunoblot analysis of purified Mic10 dissolved in different detergents, as indicated, C. BN-PAGE analysis of Mic10 dissolved in the indicated detergents and stained with Coomassie brilliant blue.

\subsubsection{Mic10 molecules are in close proximity in model membranes}

As a next step, we tested the oligomerization ability of Mic10 in model membranes. To this end fluorescently labeled Mic10-Alexa-488donor and Mic10-Alexa-568 acceptor were incorporated and co-incorporated into liposomes and subjected to FRET ex- 
periments. Efficient emission spectra were recorded for liposomes containing coincorporated Mic10 molecules, verifying that Mic10 molecules are in close proximity in model membranes (Figure 4.17).

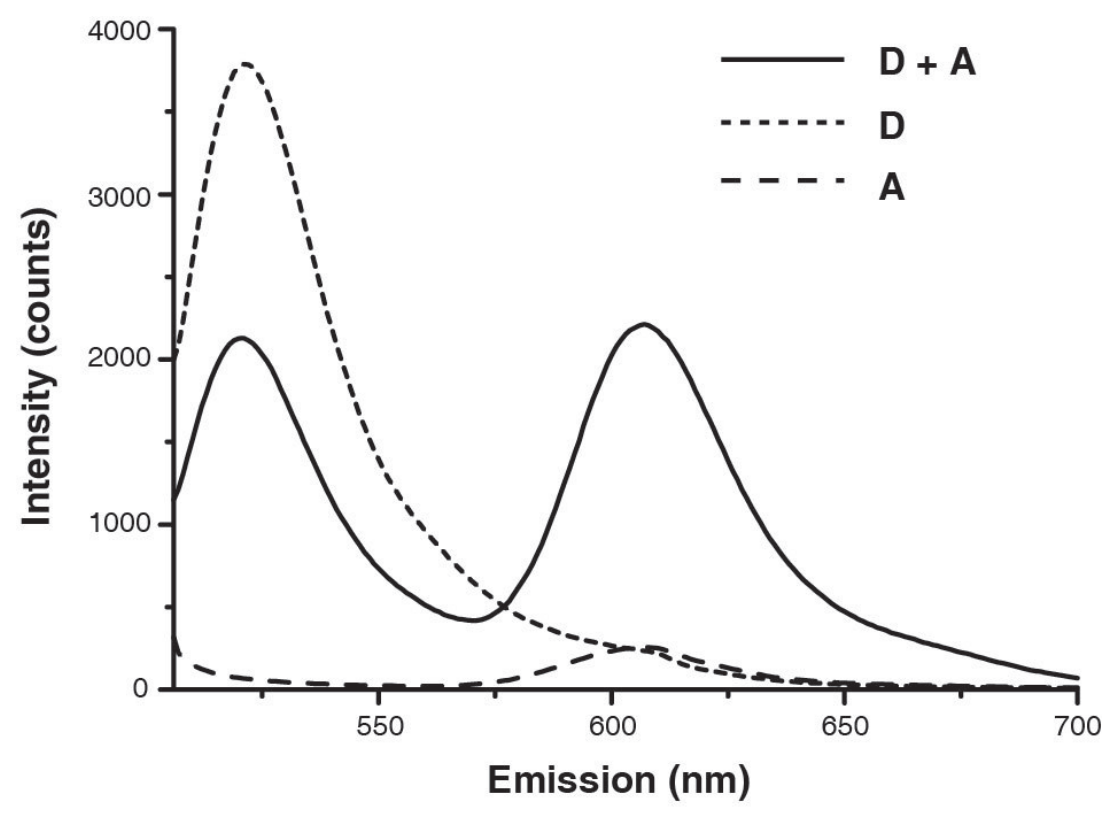

Figure 4.17: Mic10 molecules are in close proximity in LUVs. FRET spectra of fluorescently labeled Mic10 incorporated into liposomes. A corresponds to acceptor-labeled Mic10, D to donor-labeled and D + A to donor- and acceptor-labeled. The measurement was carried out by Niels Denkert from our group.

\subsubsection{Glycine-rich motifs are important for Mic10 oligomerization}

To test the importance of the glycine-rich motifs for Mic10 oligomerization, GxxxG motifs in both transmembrane domains were analyzed. To this end, a number of mutants were designed, where glycines were substituted by alanine. These mutant variants of Mic10 were purified to homogeneity from E.coli cells as described in section 4.1.1 and subjected to SDS-PAGE analysis. In comparison to the WT, the mutants Mic10 ${ }^{\mathrm{G} 50 \mathrm{~A}}$ and Mic10 ${ }^{\mathrm{G} 52 \mathrm{~A}}$ from the first and Mic10 $10^{\mathrm{G} 72 \mathrm{~A}}$ and Mic10 $10^{\mathrm{G} 74 \mathrm{~A}}$ from the second glycine-rich motifs displayed reduced numbers and intensity of high molecular weight oligomers (Figure 4.18 A, lanes 3, 4, 6, 7 and 1, respectively). 
Interestingly, a double mutant within the first transmembrane domain Mic $10^{\mathrm{G} 50 / 52 \mathrm{~A}}$ showed a striking disruption of oligomers, while Mic10 $172 / 74 \mathrm{~A}$ from the second one exhibited a similar effect to the single mutant variants (Figure 4.18 A, lane 10).

A

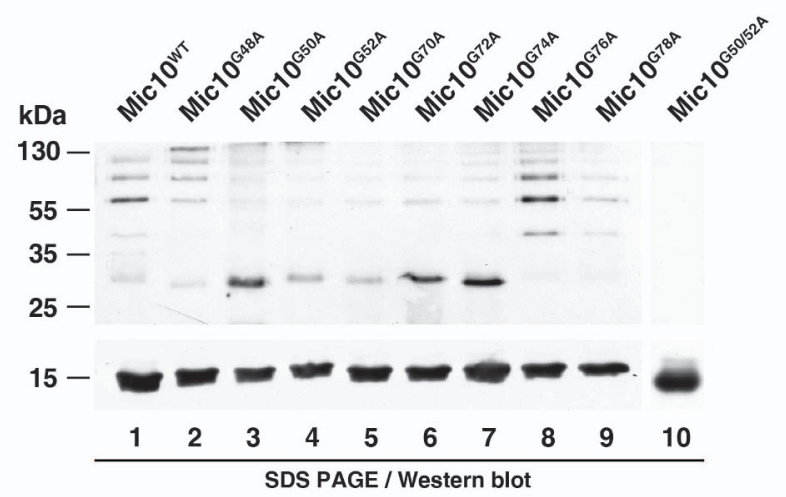

B

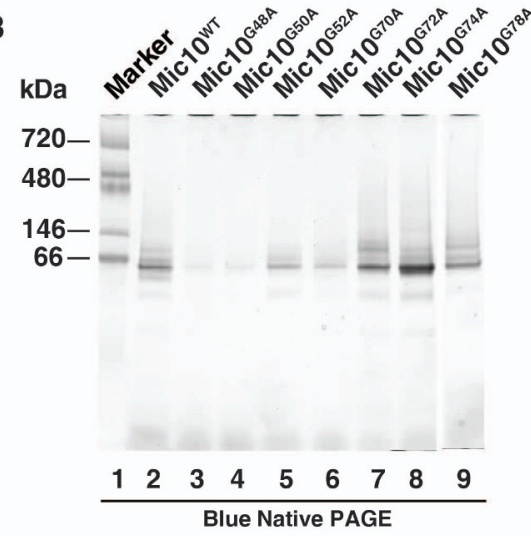

C

D

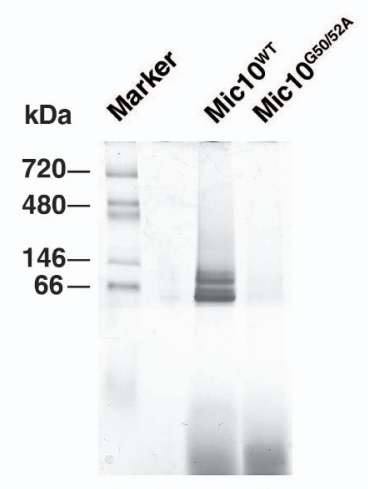

$\begin{array}{llll}1 & 2 & 3 & 4\end{array}$

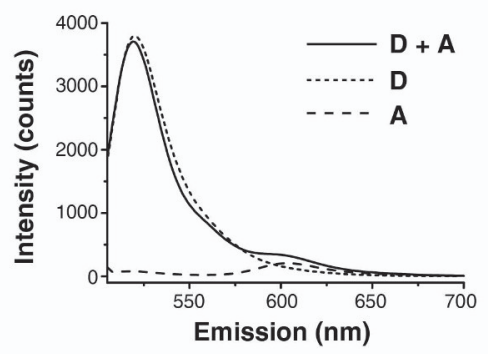

Figure 4.18: Mic10 forms oligomers via its glycine-rich motifs. A. SDS-PAGE and B. and C. BN-PAGE analysis of purified Mic10 glycine mutants as indicated, D. FRET spectra of fluorescently labeled Mic10 ${ }^{\mathrm{G} 50 / 52 \mathrm{~A}}$ incorporated into liposomes (descriped in section 4.3.2). The FRET measurement was carried out by Niels Denkert.

In order to further validate decreased oligomerization, mutants solubilized in mild detergents were analyzed by BN-PAGE. Interestingly, the single mutants Mic10 ${ }^{\mathrm{G} 48 \mathrm{~A}}$, Mic10 $10^{\mathrm{G} 50 \mathrm{~A}}$ and Mic10 ${ }^{\mathrm{G} 52 \mathrm{~A}}$ displayed significant, and the double mutant Mic10 $10^{\mathrm{G} 50 / \mathrm{G} 52 \mathrm{~A}}$ drastic loss of oligomers (Figure 4.18 B, lanes 3, 4 and 5; C, lane 4).

FRET experiments were performed for fluorescently labeled Mic10 G50/52A incorporated into liposomes (as for Mic10 ${ }^{\mathrm{WT}}$ in section 4.3.2). Almost no FRET signal 
was detected for this mutant, confirming that Mic10 $10^{\mathrm{G} 50 / 52 \mathrm{~A}}$ molecules are not in close proximity in biological membranes. Thus, Mic10 forms homo-oligomers via glycinerich motifs.

\subsection{Oligomerization of Mic10 is a prerequisite for membrane bending in vitro}

In order to test whether homo-oligomerization of Mic10 can be attributed to its membrane deformation ability, effects of Mic10 $0^{\mathrm{G} 50 / 52 \mathrm{~A}}$ on model membranes were analyzed.

A

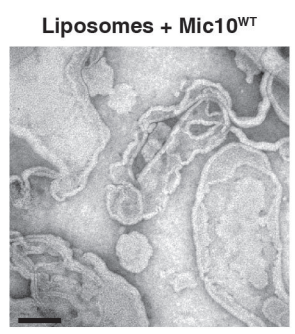

(1)
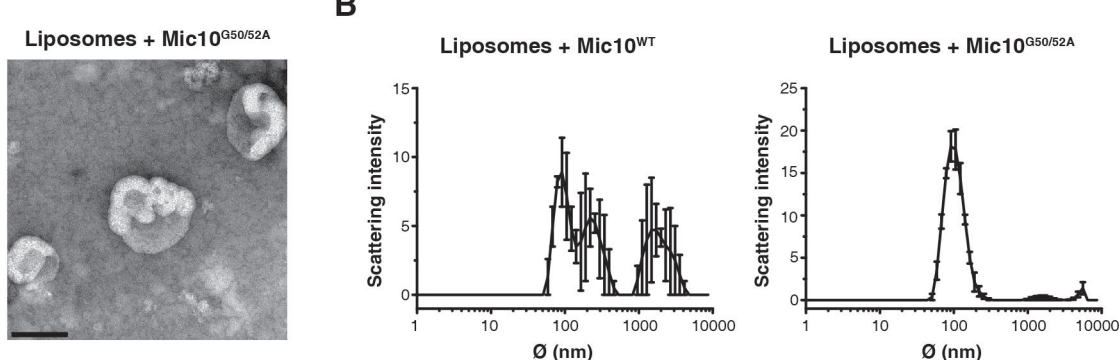

C

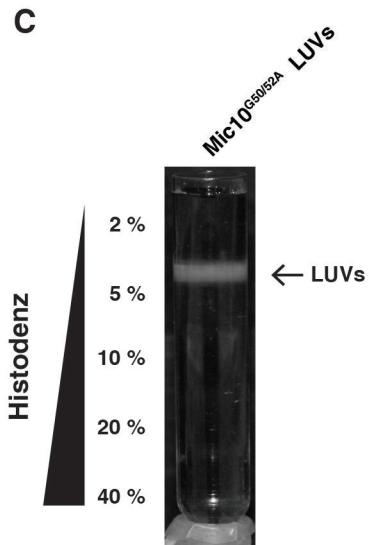

D

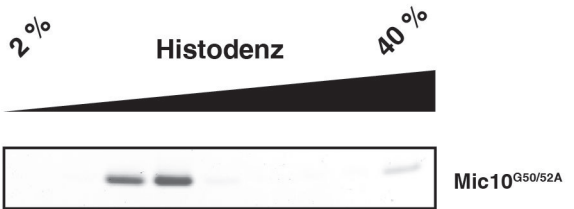

Figure 4.19: Mic10 ${ }^{\text {G50/52A }}$ fails to curve model membranes in vitro. A. Electron microscopy images of Mic10-contanining LUVs (left) (see also 4.6 A) and Mic10 ${ }^{\text {G50/52A }}$-containing LUVs (right), B. Average size-distribution profile of proteoliposomes from A measured by DLS, C. Flotation assay of Mic10 G50/52A -incorporated liposomes separated on a discrete Histodenz gradient (see Figure 4.5 A (left) for the wild type protein), D. SDS-PAGE analysis of fractionated gradient from $\mathbf{C}$. 
For this purpose, Mic10 $10^{\mathrm{G} 0 / 52 \mathrm{~A}}$ was incorporated into LUVs. Incorporation success was monitored by flotation assay, as for Mic10 WT. Interestingly, the flotation pattern of Mic10 $10^{\mathrm{G} 0 / 52 \mathrm{~A}}$-containing liposomes was very similar to that of the empty and Tim23-containing LUVs, indicating that the mutant has no apparent influence on the morphology of LUVs (Figure 4.19 $\mathrm{C}$ and $\mathrm{D}$ and Figure 4.5). To further validate the data, the proteoliposomes were analyzed by electron microscopy. EM revealed that liposomes had round shapes similar to empty or Tim23-incorporated vesicles, indicating that Mic10 $10^{\mathrm{G} 0 / 52 \mathrm{~A}}$ failed to induce membrane curvature in LUVs (Figure 4.19 A (right) and Figure 4.6 B, C and D). Moreover, DLS analysis of these proteoliposomes did not exhibit a wide size-distribution profile as was observed for Mic10and BAR domain-containing vesicles (Figure 4.19 B and Figure 4.7 A and E).

In conclusion, oligomerization of Mic10 is necessary for its membrane-bending activity in vitro.

\subsection{Mic10 oligomerization is essential for normal cristae junction formation in vivo}

Localization studies of the MICOS complex in mitochondria revealed that Mic10 is highly enriched at CJs (Harner et al., 2011). Saccharomyces cerevisiae mitochondria in the absence of Mic10 exhibit abnormal inner mitochondrial membrane architecture with a reduced number of cristae junctions (Hoppins et al., 2011; van der Laan et al., 2012). Moreover, our in vitro studies (see section 4.4) revealed that the oligomerization of Mic10 is important for its membrane-bending activity. In order to test that oligomerization of Mic10 is important for the maintenance of the inner mitochondrial membrane organization, the effects of the oligomerization-disruptive mutant Mic $10^{\mathrm{G} 50 / 52 \mathrm{~A}}$ were analyzed on yeast IM. To this end, a plasmid containing the MIC10 ${ }^{\text {G50/52A }}$ gene was introduced into mic10 $\Delta$ yeast cells and the cells were subjected to EM analysis. Interestingly, mitochondria containing Mic10 G50/52A displayed very similar phenotypes to the mitochondria of mic $10 \Delta$ cells with around $50 \%$ (60\% for mitochondria of mic $10 \Delta$ cells) altered cristae morphology and a highly decreased number of CJs (Figure 4.20). The steady-state level of Mic10 G50/52A was comparable to WT protein levels from WT cells as well as from mic10 $\Delta+$ WT cells (Figure 4.21 A). 


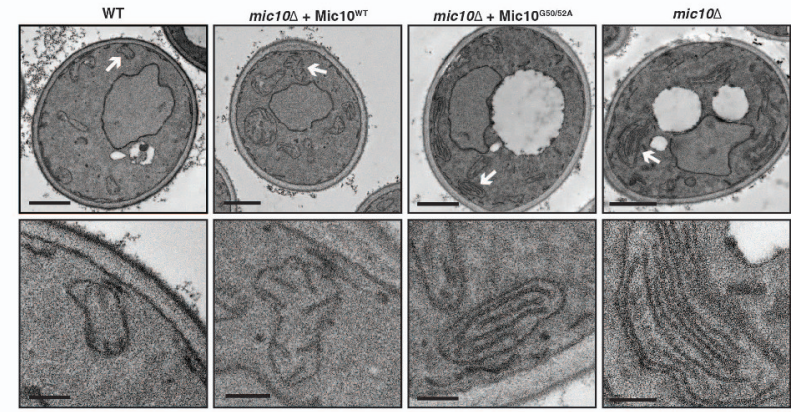

B
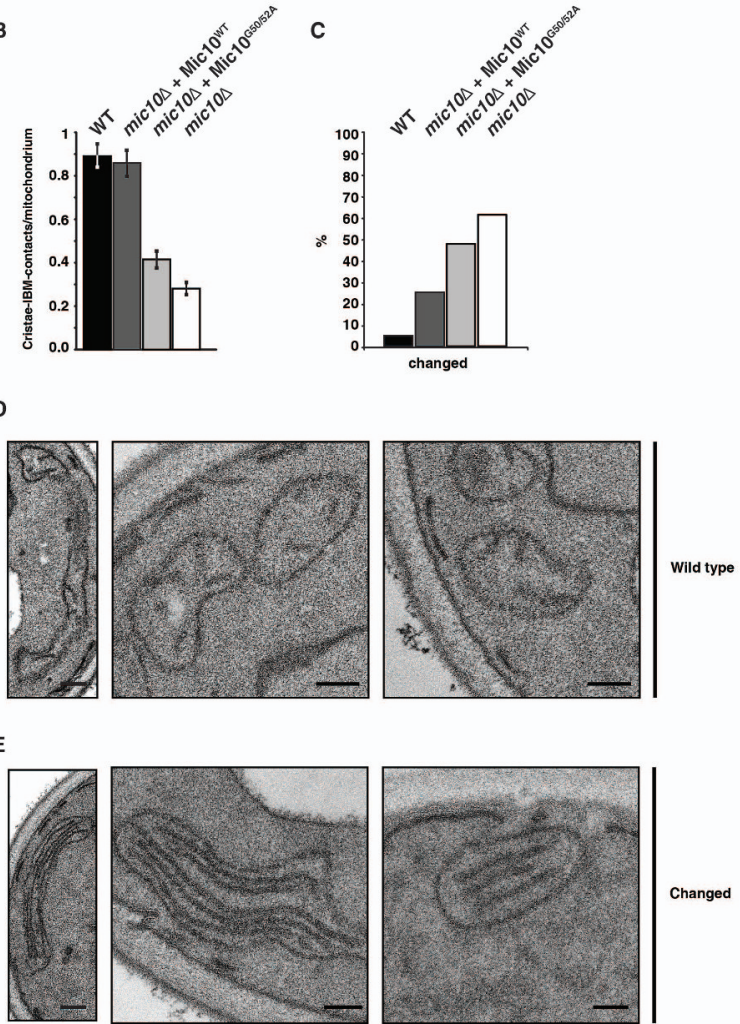

Figure 4.20: Mic10 oligomerization disruption causes the abnormal IM morphology in yeast. A. (From left to right) EM micrographs of mitochondria from WT S. cerevisiae, mic10 $\Delta$ supplemented with Mic10 ${ }^{\mathrm{WT}}$, mic10 $\Delta$ supplemented by Mic $10^{\mathrm{G} 50 / 52 \mathrm{~A}}$ and mic10 $\Delta$ cells, B. Quantification of CJs per mitochondrion as detected in cell section. Error bars represent SEM, C. quantitative evaluation of the inner mitochondrial membrane ultrastructure (see D), D. Images of mitochondria to distinguish between wild type (upper panel) and changed (lower panel) inner membrane morphology. The images were taken by Dr. Daniel C. Jans, Max Planck Institute for Biophysical Chemistry, Göttingen, Germany. 
A

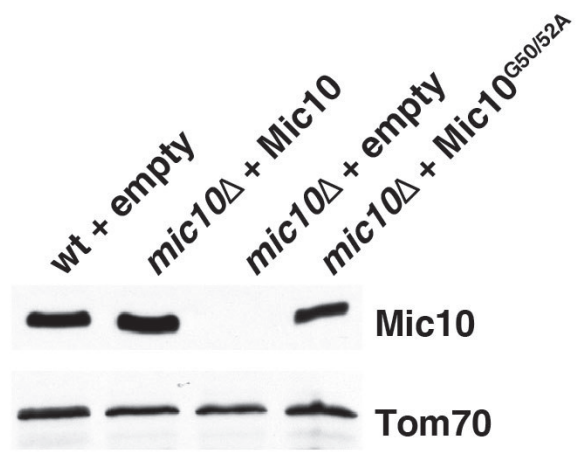

B

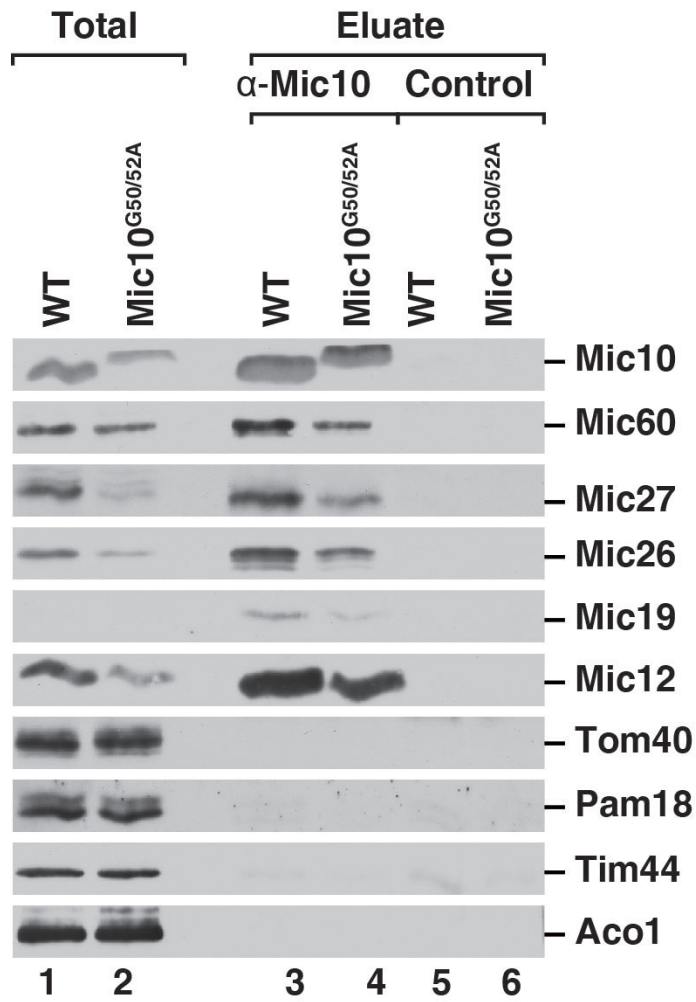

Figure 4.21: Mic10 ${ }^{\mathrm{G} 50 / 52 \mathrm{~A}}$ is a constituent of the MICOS complex. A. Steady-state levels of different Mic10 variants expressed in yeast cells as indicated, $\mathbf{B}$. Co-isolation of MICOS subunits with WT Mic10 (lane 3) and Mic10 G50/52A (lane4). The co-immunoprecipitation experiment B was performed by Dr. Christian Schulz.

Overall, our data suggest that Mic10 is essential for inner mitochondrial membrane formation and maintenance. Mitochondria from mic10 $\Delta$ cells expressing WT Mic10 showed a mild phenotype with $80 \%$ morphological similarity to the wild-type cells (Figure 4.20). To exclude the possibility that the morphological changes in the IM are not due to the Mic10 $0^{\mathrm{G} 50 / 52 \mathrm{~A}}$ mislocalization, the composition of the MICOS complex in mitochondria containing the Mic $10^{\text {G50/52A }}$ mutant was analyzed by co-immunoprecipitation. All MICOS subunits were specifically and efficiently precipitated as for the WT MICOS composition, demonstrating that Mic $10^{\mathrm{G} 50 / 52 \mathrm{~A}}$ is normally integrated into the MICOS complex (Figure 4.21 B, lanes 3 and 4). 


\subsubsection{Mic10 oligomerization disruption is not lethal}

Previous studies in yeast revealed that the absence of Mic10 is deleterious for cells when grown on a non-fermentable carbon source (Alkhaja et al., 2012). In order to examine whether expression of Mic10 $10^{\mathrm{G} 0 / 52 \mathrm{~A}}$ in yeast cells might have an effect on cell viability, we analyzed the growth phenotype of mic10 $\Delta$ cells expressing Mic10 ${ }^{\mathrm{G} 50 / 52 \mathrm{~A}}$ on fermentable and non-fermantable carbon sources at different temperatures. mic $10 \Delta+$ Mic10 ${ }^{\mathrm{G} 50 / 52 \mathrm{~A}}$ cells were grown, similar to mic10 $\Delta$ and WT cells, on glucose at all experimental temperatures. The growth on glycerol and lactate at low temperature was reduced but not as strongly as the growth of mic10 $\Delta$ (Figure 4.22).

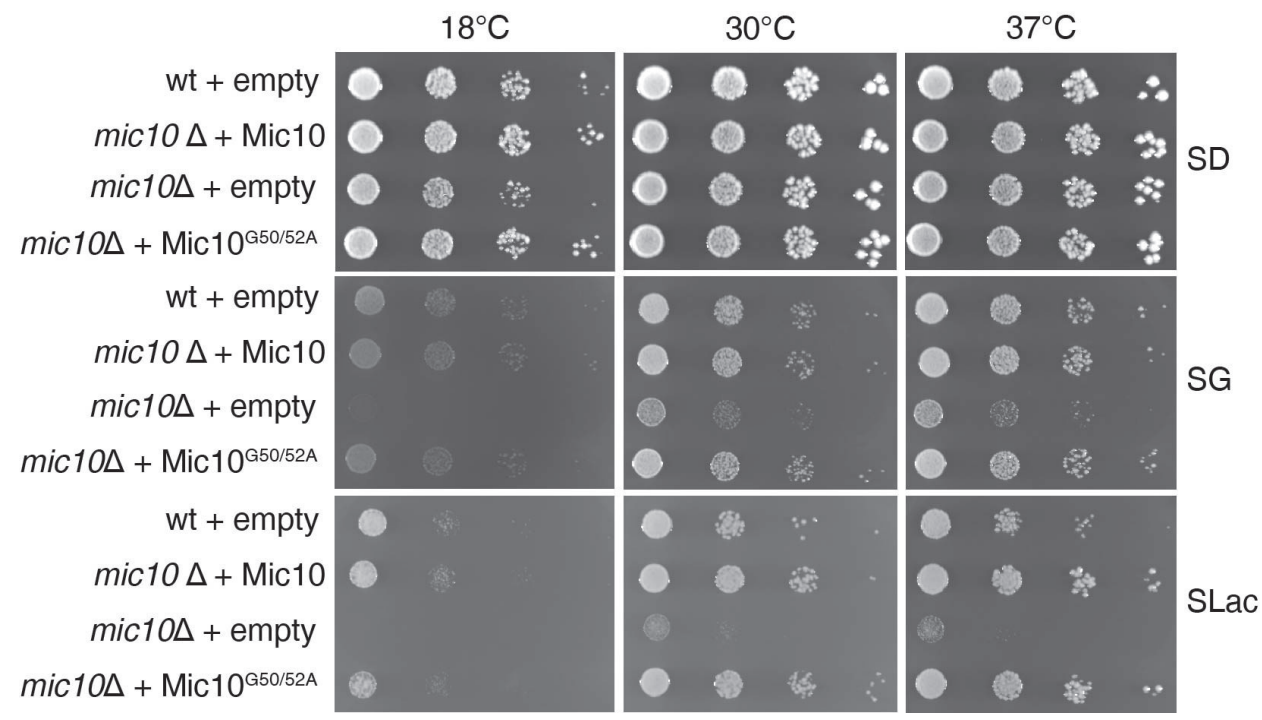

Figure 4.22: Mic10 oligomerization is not essential for cell viability - (wt + empty) - BY4741 yeast cells transformed with empty plasmid, (mic10 $\Delta+$ empty) - BY4741 yeast cells carrying a chromosomal deletion of MIC10 transformed with empty plasmid, (mic10 + Mic10) - BY4741 yeast cells carrying a chromosomal deletion of MIC10 transformed with Mic10encoding plasmid and (mic10 $\Delta+$ Mic10 ${ }^{\mathrm{G} 50 / 52 \mathrm{~A}}$ ) - BY4741 yeast cells carrying a chromosomal deletion of MIC10 transformed with Mic10 $0^{\mathrm{G} 50 / 52 \mathrm{~A}}$ encoding plasmid. These cells were spotted in 10-fold serial dilutions on selective glucose, glycerol or lactate plates and growth characteristics were monitored at $18^{\circ} \mathrm{C}, 30^{\circ} \mathrm{C}$ and $37^{\circ} \mathrm{C}$. 


\section{Discussion}

Mitochondrial inner membrane subcompartmentalization into different morphological regions is believed to be essential for proper functioning of the eukaryotic cell. In the last decade, a number of membrane proteins and protein complexes have emerged which are implicated to be involved in inner mitochondrial membrane morphology generation and maintenance. Although evidence suggests that these proteins play a role in IM organization, in vitro and structural data about their direct involvement in inner mitochondrial membrane shaping is still missing. Furthermore, it is not certain that all the molecular players involved in this process are already identified.

Interestingly, most of the proteins which are believed to sculpt the cristae membranes are integral membrane proteins. One of the main challenges the field constantly faces is the handling of the integral membrane proteins. This is also a reason for the current very limited understanding of the molecular mechanism of membrane sculpting by integral membrane proteins and thus cristae generation. In contrast, the modes of action of soluble membrane curvature inducing peripheral membrane proteins have been rather well-described.

In this study, I took a combination of in vitro bottom-up and in organello approaches to investigate the molecular players and the underlying mechanisms in cristae membrane shaping.

\subsection{Mic10 tubulates round liposomes}

Since the recognition of cristae junctions as structural organizers of the mitochondrial inner membrane (Mannella, 2006) and the discovery of their role in cellular physiology (Scorrano et al., 2002), more attention has been directed on unraveling the molecular players involved in CJ formation and stabilization. Discovery of the evolutionary conserved protein complex MICOS brought a new perspective in 
understanding this process (Hoppins et al., 2011; Harner et al., 2011; von der Malsburg et al., 2011; Alkhaja et al., 2012). Although a great deal of evidence obtained in recent years from cell biology and biochemical studies suggest the importance of MICOS in CJ maintenance, in vitro membrane deformation studies are necessary to unambiguously show the role of MICOS in CJ shaping.

In order to study the direct role of the MICOS complex in CJ formation, I investigated the function of the MICOS subunit, Mic10. Mic10 is one of the core components of the complex and along with Mic60, shows the strongest abnormal inner mitochondrial membrane phenotype upon depletion (Alkhaja et al., 2012; Barbot et al., 2015; Bohnert et al., 2015b). To this end, I recombinantly expressed S. cerevisiae Mic10 and purified it from E.coli cells. Using recombinantly expressed membrane proteins has an advantage with respect to obtaining enough sample. However, recombinantly expressed hydrophobic proteins, which require harsh detergents for purification, may provide misleading results when addressing their functional properties. Nonetheless, the purification protocol for Mic10 described in 3.2.3.6 was previously successfully applied for isolation of a number of mitochondrial membrane proteins, which were shown to be functionally competent after incorporation into liposomes (Hill et al., 1998; Kovermann et al., 2002; Meinecke et al., 2006; van der Laan et al., 2007).

As model membrane systems, large and giant unilamellar vesicles were chosen, simply because upon external stimuli, the morphological changes can be directly visualized.

The incorporation of Mic10 into LUVs led to morphological changes of liposomes. Spherical lipid vesicles were converted into tubular membrane structures as visualized by EM. The formation of tubules in vitro from relatively flat membranes is a common characteristic of many proteins that are involved in cellular membrane remodeling e.g. proteins involved in clathrin-mediated endocytosis and ER shaping proteins (Sackmann, 2014; McMahon and Boucrot, 2011). Interestingly, Mic10-containing membrane tubules depicted diameters between 10 and $30 \mathrm{~nm}$, which is very similar to the diameter of CJs (Frey and Mannella, 2000). Moreover, over the course of a day the Mic10-containing liposomes turned into larger vesicular structures with internal membrane structures. Similar time-dependent effects were also observed for other membrane curving proteins such as the ER shaping protein, Yop1p. However, Yop1p-containing vesicles were converted into longer tubules 
than the structures observed in this study ( $\mathrm{Hu}$ et al., 2008). Although there is no solid explanation for this effect, it is conceivable to speculate that the appearance of the heterogeneous membrane structure, over time, can be attributed to the different oligomeric state of Mic10. In fact, in our observations of Mic10 structure using EMmicroscopy, we continuously observed Mic10 molecules self-assembled into heterogeneous sized oligomers. Moreover, as recently shown by different experimental approaches MICOS is a highly dynamic complex. In addition to a holocomplex, MICOS can also occur as different sub-complexes and the amount of each subunit in the holocomplex and sub-complexes largely differ from each other (Harner et al., 2014; Friedman et al., 2015; Bohnert et al., 2015b; Guarani et al., 2015).

It has to be mentioned that the in vitro approach includes a detergent mediated reconstitution procedure, which could lead to experimental artifacts. To verify that the membrane morphology changes are indeed attributed to Mic10, rigorous control experiments were carried out. As negative controls mock- and Tim23-incorporated liposomes were used. Indeed, these preparations did not lead to any structural changes of liposomes. This was not surprising as Tim23 is a channel-forming subunit of the TIM23 translocase of the inner mitochondrial membrane and it is not involved in membrane remodeling processes (Truscott et al., 2001). In contrast, Endophilin BAR domain converted liposomes into tubules, an effect which is in accordance with the proteins action on membranes (Meinecke et al., 2013).

When the effect of Mic10 in membranes was examined in bulk, Mic10-containing liposomes showed large-scale shifts in size distribution, whereas mock- and Tim23incorporated vesicles retained their original size of $100 \mathrm{~nm}$ diameter. This further validated the observation that Mic10 has the ability to deform membranes. Mic10containing samples also revealed vesicles with the size of $100 \mathrm{~nm}$ diameter and the same was observed for liposomes in the presence of Endophilin BAR domain. Moreover, Mic10-containing vesicles were observed to be present at various density layers of the Histodenz gradient, including the density layer where the mock- and Tim23-incorporated liposomes solely accumulated as a result of flotation. Therefore, it was reasoned that Mic10-containing vesicles might acquire different morphologies and sizes. In order to test this assumption, the density layers of the floated Mic10containing vesicles were dissected and analyzed using EM. However, because of the strong Histodenz background, even after dialysis, it was not possible to visually examine the samples. Nonetheless, analysis of the vesicle size distribution profile 
from the various dissected layers revealed that Mic10-containing vesicles acquire different sizes. Even though it is debatable whether the observed heterogeneous size of Mic10-containing vesicles can be directly correlated to their distinct morphologies, the experimental evidence supports this concept. In particular, proteoliposomes from bottom layers (correlating to higher density) depicted pronounced size shifts, whereas proteoliposomes from upper layers (correlating to lower density, to which non-treated LUVs migrate) showed a fairly uniform size distribution profile, with an average $100 \mathrm{~nm}$ diameter. As the mock- and Tim23-incorporated vesicles were also found at the upper layers of the density gradient, it is reasonable to propose that the Mic10-incorporated liposomes from upper layers should also retain round shapes as do the controls. This is in agreement with the observation that non-floating Mic10containing vesicles also showed round membrane morphologies in addition to tubular structures when examined using EM. Importantly, the dissected layers analyzed by SDS-PAGE revealed that Mic10 was present in all liposome-containing layers. It was highly enriched in the middle and, to a lesser extent, bottom layers, whereas in the upper layers it was less pronounced. The precise amount of protein integrated into liposomes at the different layers is extremely difficult to examine, however, we observed that Mic10 membrane activity is concentration dependent (below $200 \mathrm{nM}$ no liposome morphological changes were observed). It is therefore tempting to propose that the liposomes from upper layers contain insufficient amounts of Mic10 and hence, this is the reason for the observed round membrane morphologies.

To further examine that liposome morphology changes are indeed attributed to membrane integrated Mic10 and are not depend on the model membrane type, the effects of Mic10 on GUVs were addressed. LUVs and GUVs have very different physico-chemical properties. Interestingly, the incorporation of Mic10 into GUVs also led to drastic morphological changes of liposomes as observed using confocal fluorescence microscopy. The GUVs depicted the internal tubular and vesicle structures. What is noteworthy here is that all internal membrane structures were evenly decorated by Mic10. The same characteristics was observed after analysis of immunogold decorated Mic10-containing LUVs. Therefore, it would be interesting to address if the IM lipid cardiolipin plays a role in clustering Mic10 at highly curved membrane regions. 


\subsection{Mic10 membrane curving mechanism}

As no high resolution structural information about Mic10 is available, to unravel the molecular mechanism by which Mic10 shapes membranes, I determined the membrane topology of Mic10 in mitochondria. Topology studies revealed that two transmembrane domains of Mic10 span the IM in a hairpin topology with the $\mathrm{C}$ - and $\mathrm{N}$-termini exposed towards the intramembrane space (Figure 4.13) (Barbot et al., 2015). Interestingly, this membrane topology was confirmed by parallel studies (Bohnert et al., 2015b). The N-terminal transmembrane domain of Mic10 is unusually long (24 amino acids, whereas the normal length is 20 amino acids). In order to avoid a hydrophobic mismatch, I propose that Mic10 spans the IM in a tilted orientation. The logical consequence would be an asymmetric wedge-like topology within the IM, that would lead the displacement of lipids at the IMS side of the lipid bilayer and thereby induce the curvature observed at CJs (Figure 5.1). The suggested model is similar to the membrane curving model for ER shaping proteins, reticulons and Yop1p/DP1, that have a similar membrane topology to Mic10 (Voeltz et al., 2006).

Protein induced membrane deformation is often a consequence of oligomerization or clustering of proteins. Mic10 contains highly conserved glycine-rich (GxxxG/ GxGxG) motifs in both transmembrane domains (Alkhaja et al., 2012; Zerbes et al., 2012b; Barbot et al., 2015; Bohnert et al., 2015b). Such glycine-rich motifs are important for transmembrane helix packing (Russ and Engelman, 2000). A number of proteins have been reported whose oligomerization depends on their glycinerich motifs (Arnold et al., 1998; Vonck et al., 2002; Wagner et al., 2009; Alavian et al., 2014; Demishtein-Zohary et al., 2015). As confirmed by SDS- and BN-PAGE analysis, detergent solubilized Mic10 indeed forms homo-oligomers mediated by its GxxxG motifs. Furthermore, Mic10 molecules cluster after reconstitution into model membranes as revealed by FRET studies (Barbot et al., 2015). The parallel studies also reported about the Mic10 oligomerization ability by analyzing the native yeast Mic10 by BN-PAGE (Bohnert et al., 2015b). Importantly, the glycine mutant variants of Mic10, lost the ability to oligomerize, failed to tubulate liposomes (Barbot et al., 2015) and, more importantly, after expressing in yeast cells, failed to maintain normal cristae morphology (Barbot et al., 2015; Bohnert et al., 2015b).

The observation that Mic10 oligomers generate highly curved membranes is in 


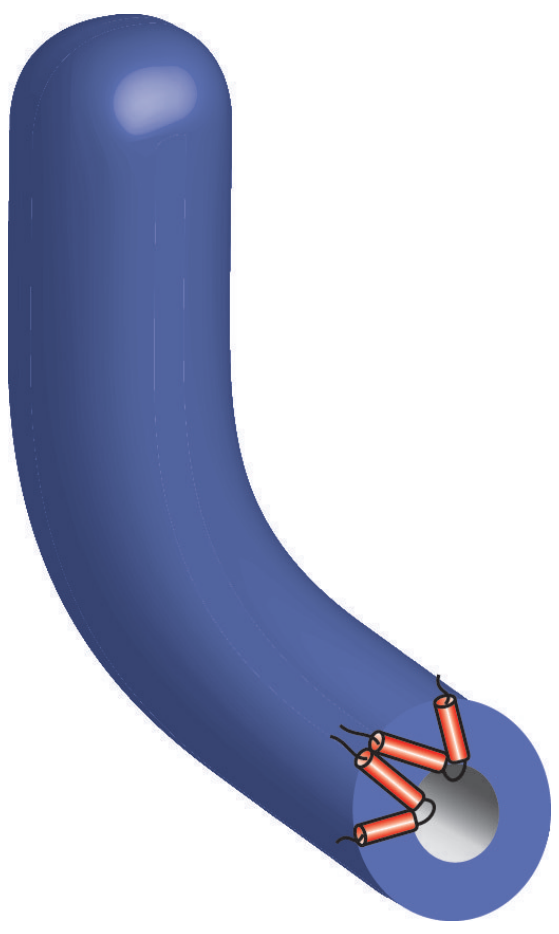

Figure 5.1: Model of tubular cristae junction formation by Mic10. Mic10 adopts an asymmetric wedge topology in the membrane. The highly conserved transmembrane regions lead to Mic10 homo-oligomerization. Mic10 homo-oligomers might form a scaffold around the membrane tubule. Adapted from (Barbot and Meinecke, 2016, in revision).

accordance with the energy demands to bend membranes. Using the classical Canham, Helfrich, and Evans theory, the energy cost per area of bending bilayer to create curved tubular structure can be estimated as $\mathrm{G}_{\text {bending }}=(8 \pi \kappa) /(2 \pi \mathrm{rl})$, where $\kappa$ is the bending rigidity of the membrane and $r$ and $I$ are the generated tubule radius and length, respectively (Canham, 1970; Helfrich, 1973; Stachowiak et al., 2013). The bending rigidity for soft fluid membranes was calculated to be $\sim 10 \mathrm{~K}_{B} \mathrm{~T}$ (Evans and Rawicz, 1990). The kinetic parameters of Mic10 as dissociation constant of transmembrane helix oligomerization are not known. However, the free energy of dimer dissociation for other glycine-rich motif containing proteins, like glycophorin A was measured to be $\sim 15 \mathrm{~K}_{\mathrm{B}} \mathrm{T}$ (or $9.0 \mathrm{kcal} / \mathrm{mol}$ ). If we assume a comparable value for Mic10, the energy cost of membrane deformation could be easily provided by Mic10 oligomer formation.

In conclusion, on the basis of the observations from this study regarding Mic10 
hairpin topology, oligomerization ability and necessity, it is reasonable to propose that Mic10 shapes the inner mitochondrial membrane at cristae junctions by a combinatorial mechanism of membrane wedging and scaffolding (Figure 5.1) (Barbot et al., 2015).

\subsection{Possible role of other MICOS subunits in cristae sculpting}

The MICOS complex consists of at least six different subunits and most of them appear to be crucial for inner mitochondrial membrane maintenance (Hoppins et al., 2011; Harner et al., 2011; von der Malsburg et al., 2011; Alkhaja et al., 2012; Barbot et al., 2015; Bohnert et al., 2015b). Nonetheless, with the exception of Mic10, their exact role in $\mathrm{CJ}$ formation has not been addressed.

Mic60 is a core subunit of the MICOS complex along with Mic10. Similar to Mic10, depletion of Mic60 leads to abnormal IM morphologies with a loss of CJs (van der Laan et al., 2012). Overexpression of Mic60 in yeast cells leads to highly branched cristae membranes (Rabl et al., 2009; Bohnert et al., 2015b). The effects on membrane morphology upon overexpression of MICOS single subunits were only observed for Mic60 and Mic10, leading to the assumption that, like Mic10, Mic60 might also directly shape the cristae membranes (Bohnert et al., 2015b). How could Mic60 shape CJs? Mic60, has also been reported to form homo-oligomers (John et al., 2005; Rabl et al., 2009; Herrmann, 2011), however unlike Mic10, it only contains one transmembrane domain. Caveolin, an integral membrane protein which is required for caveoli formation in the plasma membrane, also forms oligomers and is suggested to acquire a hairpin topology in the membrane with its single hydrophobic domain (Ostermeyer et al., 2004). However, the transmembrane domain of caveolin is 34 amino acids long and might induce membrane deformation in a similarly way to Mic10 or ER shaping proteins. The transmembrane domain of Mic60 is 20 amino acids long and therefore is unlikely to adopt an asymmetric wedge in cristae membranes. Earlier studies of Mic60 revealed that the absence of different regions of the IMS domain strongly affects MICOS complex integrity and IM morphology (Zerbes et al., 2012a) pointing to their possible role in CJ shaping. Based on these observations, Mic60 might directly bend cristae membranes with its IMS domain rather than with the transmemrbane domain. The generation of membrane curvature by the 
IMS domain of Mic60 could be achieved by a protein crowding mechanism. Protein crowding by protein-protein interactions has been shown to be sufficient for bending membranes regardless of how proteins are recruited to the membrane (Stachowiak et al., 2012). Thus, membrane bending by a crowding mechanism solely depends on the local protein concentration in the vicinity of membranes (Stachowiak et al., 2012). Taking this into account, as well as that Mic60 is highly enriched at CJs (Hoppins et al., 2011; Jans et al., 2013), the most likely mechanism of CJ curving by Mic60 is the protein crowding mechanism.

Overall, if above-discussed holds true, then CJs are curved by a combinatorial effort of two MICOS membrane proteins. Although the exact energetic contribution for membrane bending by each protein is not known, a computational assessment of curvature induction by crowding revealed that this mechanism has a lower curvature generation efficiency than the mechanism based on the shallow insertion of amphiphatic $\alpha$-helices (Kozlov et al., 2014). If we assume that the insertion of hydrophobic domains also has a higher curvature generation efficiency than protein crowding, then Mic10 molecules would more efficiently bend membranes than Mic60. Hence, Mic10 might play a main role in CJ formation by introducing the "main" curvature, whereas Mic60 adopts more a supportive role.

To date, the exact contribution of other MICOS subunits in physiological cristae maintenance is unclear. Based on the very limited information available they are probably indirectly involved in inner mitochondrial membrane biogenesis.

\subsection{Model of cristae membrane reconstitution}

Although there is still a long way before the mechanism of mitochondrial cristae biogenesis is thoroughly understand, based on the results obtained from this study and already identified factors, it is possible to propose a mechanistic model of cristae formation and stabilization.

The generation of mitochondrial cristae membranes requires bending of the inner mitochondrial membrane, which is a energy demanding process. According to the current findings and widely accepted models in the field of membrane biophysics and biochemistry, such bending energy can be obtained by a combinatorial effort of mitochondrial lipids and intergral membrane proteins. To date, three different pro- 
teins or protein complexes; the $\mathrm{F}_{1} \mathrm{~F}_{\mathrm{O}}$-ATP synthase, Mgm1/OPA1 and Mic10/MICOS, are believed to be designated for cristae formation. These proteins probably sculpt and maintain the highly curved cristae tips and cristae junctions in the inner mitochondrial membrane.

$F_{1} F_{O}$-ATP synthase dimers and dimer rows have been widely accepted to be involved in the formation and stabilization of the highly curved cristae tips. Conventionally, lipid monolayer bending in the direction from the heads to the tails, corresponds to a positive curvature, whereas bending in the opposite direction results in a negative curvature (Zimmerberg and Kozlov, 2006). Hence, the $F_{1} F_{O}$-ATP synthase oligomers form and stabilize the positive curvature of the inner membrane at the cristae tips when looked at from the matrix side. This is in agreement with the topology of $F_{1} F_{O}$-ATP synthase dimers on cristae tips (Strauss et al., 2008; Davies et al., 2012). Importantly, although the $F_{1} F_{O}$-ATP synthase is not only found in cristae tips, two striking findings strongly advocate its role solely in cristae tip generation. First, the dimers and dimer rows of the $F_{1} F_{O}$-ATP synthase are highly enriched at cristae tips and second, the absence of oligomerization subunits leads to the disappearance of cristae tips, whereas cristae junctions are retained (Figure 1.5) (Rabl et al., 2009; Davies et al., 2011).

In contrast to cristae tips, cristae junctions are most probably generated and maintained by two functionally and structurally different proteins or protein complexes, Mgm1/OPA1 and Mic10/MICOS (Frezza et al., 2006; Barbot et al., 2015; Bohnert et al., 2015b; van der Laan et al., 2012). This raises the question about their exact contribution to $\mathrm{CJ}$ formation. Studies from Rabl et al. suggested that cristae junctions exhibit topologically different regions with two types of membrane curvature: a negative curvature at the $\mathrm{CJ}$ base towards the inner boundary membrane and a positive curvature at the CJ neck (Figure 5.2) (Rabl et al., 2009). Based on the membrane topology of Mic10 determined in this study and by others, plus the observation that Mic10 sculpts model membranes to convert them into highly curved tubular structures, which exhibit similar dimensions as CJs, we propose that Mic10 generates the negative curvature at the base of CJs (Barbot et al., 2015; Bohnert et al., 2015b). Although there is very limited experimental evidence for direct membrane curvature induction by either forms of Mgm1/OPA1, based on its similarity to dynamin, it is tempting to speculate that Mgm1/OPA1 contributes to $\mathrm{CJ}$ formation by inducing a positive curvature at the tubular CJ necks, which are similar to the 
neck regions of to mature clathrin-coated pits (CCPs). Dynamin in CCPs is assembled into the surface of the tubule, whereas Mgm1/OPA1 is exposed to the inside of the neck region of the CJ tubule. Moreover, after pinching off the CCPs the donor membrane would ultimately adopt the initial flat morphology, hence the membrane deformation by dynamin is transient. Membrane shaping by Mgm1/OPA1 cannot be transient, as the CJs are the permanent structures. Therefore, we hypothesize that Mgm1/OPA1 stabilizes/generates CJs by forming hetero-oligomeric (complexes comprising of both soluble IMS and transmembrane domain containing variants), scaffolding complexes.

As already mentioned, mitochondria play a central role in apoptosis. The essential step in initiating mitochondrial apoptosis is the release of apoptotic proteins from the intracristae lumen to the cytosol. This requires the intensive remodeling of cristae membranes and ultimate widening of cristae junctions (Scorrano et al., 2002). The cellular consequences of OPA1 downregulation upon apoptotic stimuli resulting in the widening of cristae junctions and cytochrome $c$ release is well-known (Frezza et al., 2006), whereas the consequences of Mic10/MICOS downregulation is largely still missing. However, recent studies reported, that expression of mutant forms of the mammalian MICOS subunit, CHCHD10, lead to the disassembly of the MICOS complex and abnormal inner mitochondrial membrane architecture, with a reduced number of CJs. Furthermore, cells were protected from death by the prevention of cytochorome $c$ release upon induction of apoptosis (Genin et al., 2015). This data is in agreement with the previously reported observation that, upon permeabilization of the outer mitochondrial membrane in mic10 $\Delta$ and mic60 $\Delta$ yeast cells by the apoptotic protein Bax, the intermembrane space proteins were not released but remained trapped within the IM membrane stacks (von der Malsburg et al., 2011). Thus, two major players involved in cristae membrane generation by sculpting the CJs when absent exhibited antagonistic physiological responses upon apoptotic stimuli. From the morphological point of view, the above-discussed further emphasizes the striking differences between opa1 $\Delta$ and micos $\Delta$ IMM morphological phenotypes and supports the proposition that, both complexes are important for the formation and stabilization of CJs, which they achieve by sculpting tubular structures at their different topological regions. 

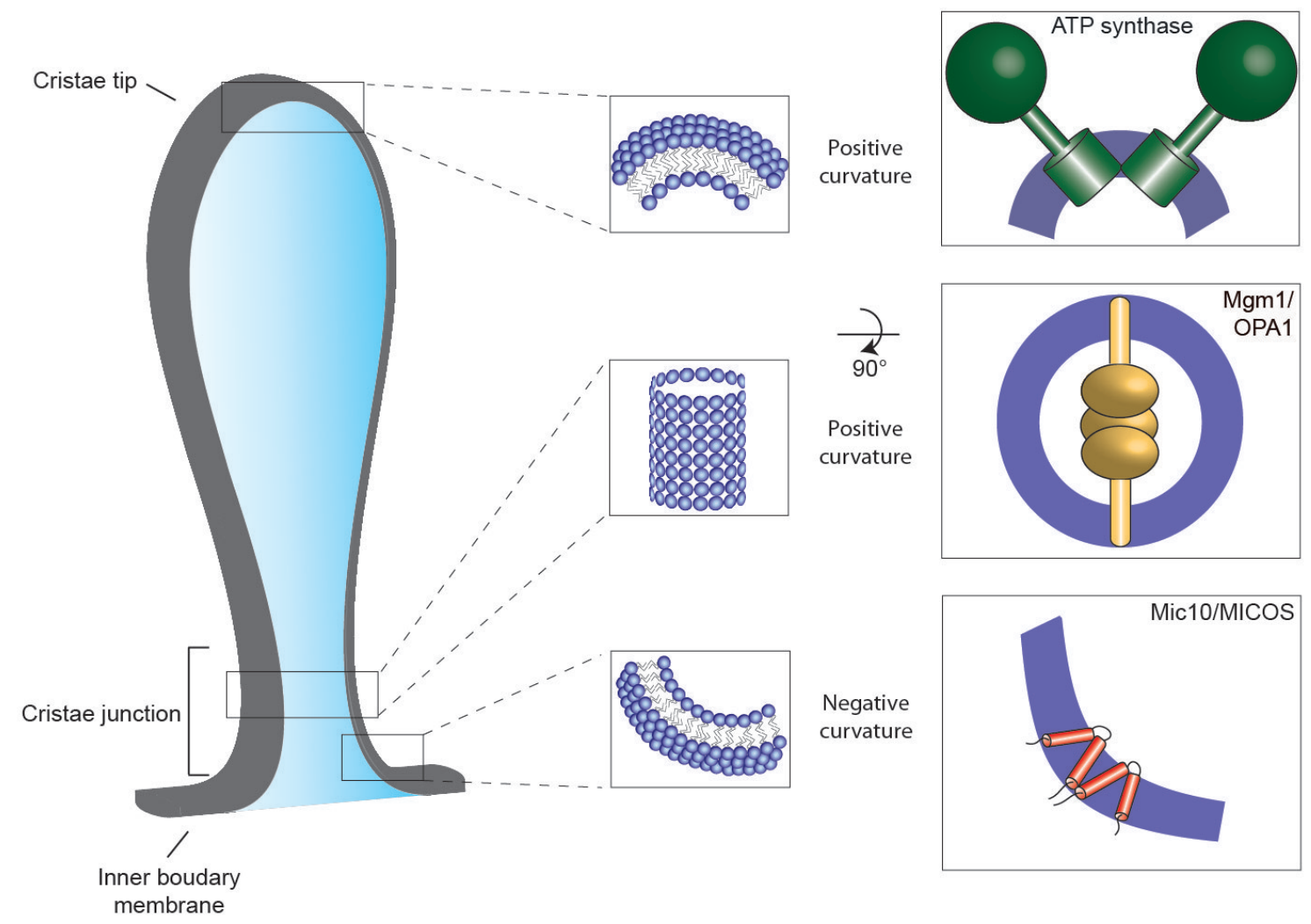

Figure 5.2: Model of cristae membrane reconsitution. The mitochondrial cristae membrane consists of different morphological regions that acquire specific high degrees of membrane curvature (depicted as side view). Cristae junctions are characterized by two different types of membrane curvature: negative, generated by Mic10/MICOS and positive, formed by Mgm1/OPA1 (shown as a cross section after $90^{\circ}$ rotation). Positive curvature of cristae tips are generated by $\mathrm{F}_{1} \mathrm{~F}_{\mathrm{O}}$-ATP synthase dimers. Adapted from (Barbot and Meinecke, 2016, in revision).

Overall, it appears that the interplay of all three complexes is necessary for normal cristae membrane formation and maintenance in eukaryotic cells. This is further underlined by the observation of genetic interactions between genes of MICOS and ATP synthase subunits, as well as OPA1 and ATP synthase subunits (Hoppins et al., 2011; Amutha et al., 2004). 



\section{Summary and Conclusion}

Within the eukaryotic cell mitochondria play a key role in many important cellular processes from energy metabolism to the regulation of apoptosis. Because of their endosymbiotic ancestry, mitochondria contain two morphologically and functionally distinct membranes. The highly convoluted inner mitochondrial membrane consists of structurally and functionally different regions, displaying different degrees of membrane curvature. To date it is widely accepted that the peculiar curved regions of the inner membrane are generated and stabilized by various inner mitochondrial membrane proteins and protein complexes.

In this thesis, one of the core subunits of the recently discovered MICOS complex, Mic10, was identified as an important molecular player in the generation and maintenance of highly curved cristae junctions. Using an in vitro membrane reconstitution bottom up approach, we unraveled Mic10's ability to directly sculpt model membranes of different physico-chemical properties.

To define the molecular mechanism of Mic10 curvature induction the topology of Mic10 in the inner mitochondrial membrane was determined. Based on this determination, it can be concluded that Mic10 contains two transmembrane helices that span the inner mitochondrial membrane in a hairpin topology, with the $C$ and $\mathrm{N}$-termini exposed towards the intermembrane space. The first transmembrane domain of the protein is unusually long. Therefore we reason that in order to avoid the hydrophobic mismatch, it spans the inner mitochondrial membrane in a tilted orientation. Such an insertion would allow the protein to occupy a larger surface at the IMS side of the inner membrane and thus, lead to membrane bending. However, structural information regarding the transmembrane helices are necessary to understand the exact molecular mechanism of membrane remodeling by Mic10.

We also showed that Mic10 forms homo-oligomers through highly conserved glycine-rich motifs, present in both transmembrane helices. The oligomerization of Mic10 is a prerequisite for membrane bending both in vitro and in vivo. 
Membrane structures generated by Mic10 display a similar dimension to the cristae junctions of the inner membrane, however they do not fully resemble the morphology of cristae membranes. Therefore, further work must be invested to determine the role of other MICOS subunits, as well as the $F_{1} F_{O}$-ATP synthase, Mgm1/OPA1 and mitochondrial lipids in the formation and maintenance of cristae structures.

In conclusion, we identified to the best of our knowledge, the first mitochondrial integral membrane protein, which sculpts the inner mitochondrial membrane at cristae junctions. The possible molecular mechanism of IM shaping at CJs by Mic10 is most probably a combination of membrane asymmetric wedging and scaffolding. 


\section{List of Figures}

1.1 The plasma membrane and membrane-bound organelles of the eukaryotic cell displaying different morphologies . . . . . . . . . . . . 2

1.2 Schematic representation of mitochondrial subcompartmentalization

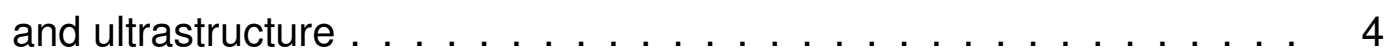

1.3 Diversity of the mitochondrial ultrastructure $\ldots \ldots \ldots \ldots$

1.4 Mitochondrial inner membrane protein organization and distribution. . 6

1.5 Role of various mitochondrial proteins in cristae morphology maintenance . . . . . . . . . . . . . . . . . 10

1.6 Composition of MICOS complex $\ldots \ldots \ldots \ldots \ldots$

4.1 Mic10 overexpression test from different $E$. coli vectors . . . . . . 50

4.2 Mic10 aggregation recovery in chaotropic agents . . . . . . . . . 50

4.3 Expression and purification of recombinant Mic10 . . . . . . . . . 51

4.4 Solubility test of Mic10 in mild detergents . . . . . . . . . . 52

4.5 Recombinantly expressed Mic10 can be reconstituted into LUVs . . . 53

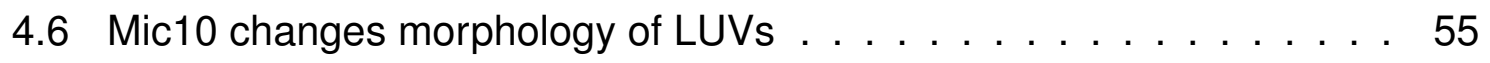

4.7 Mic10 changes the size of liposomes in bulk . . . . . . . . . 56

4.8 Size distribution profile of Mic10-containing liposomes after flotation . 57

4.9 Mic10 changes morphology of GUVs . . . . . . . . . . . 58

4.10 Mic10 is evenly distributed in liposomes . . . . . . . . . . . . 59

4.11 EM micrographs of Mic10 dissolved in detergent . . . . . . . . . 60

4.12 Sample preparation for solid state NMR . . . . . . . . . . . . 60

4.13 Putative topologies of Mic10 in the inner mitochondrial membrane . . 61

4.14 Topology mapping of Mic10 in inner mitochondrial membrane . . . . 62

4.15 In vitro Mic10 membrane topology mapping . . . . . . . . . . . 63

4.16 Mic10 forms homo oligomers . . . . . . . . . . . . . . . 65

4.17 Mic10 molecules are in close proximity in model membranes . . . . 66

4.18 Mic 10 forms oligomers via its glycine-rich motifs . . . . . . . . 67 
4.19 Mic10 $0^{\mathrm{G} 50 / 52 \mathrm{~A}}$ fails to curve model membranes in vitro . . . . . . . . . 68

4.20 Mic10 oligomerization disruption causes the abnormal IM morphology

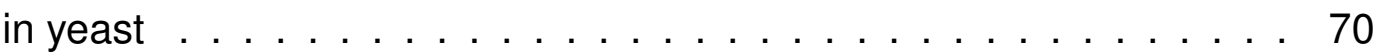

$4.21 \mathrm{Mic} 10^{\mathrm{G} 50 / 52 \mathrm{~A}}$ is a constituent of the MICOS complex . . . . . . . 71

4.22 Mic10 oligomerization is not essential for cell viability . . . . . . . . . 72

5.1 Model of tubular cristae junction formation by Mic10 $\ldots \ldots \ldots$. . 78

5.2 Model of cristae membrane reconsitution $\ldots \ldots \ldots \ldots$ 


\section{List of Tables}

3.1 Reagents and their suppliers used in this study . . . . . . . . . 23

3.2 Commercial kits and their suppliers . . . . . . . . . . . . . 25

3.3 Lipids and their manufacturers $\ldots \ldots \ldots \ldots \ldots$

3.4 Antibodies used in this study $\ldots \ldots \ldots \ldots \ldots$

3.5 E.coli strains . . . . . . . . . . . . . . . . . . . . . . . 27

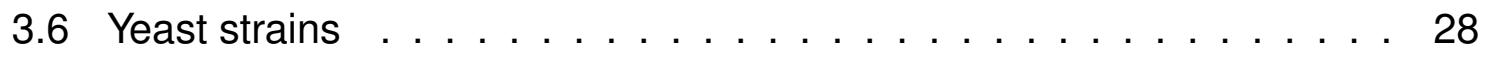

3.7 E.coli plasmids . . . . . . . . . . . . . . . . . . . . . . . . . . . . . 29

3.8 Yeast plasmids $\ldots \ldots \ldots \ldots \ldots$

3.9 Instruments and their manufacturers . . . . . . . . . . . . 30

3.10 Softwares and their manufacturers $\ldots \ldots \ldots \ldots$. . . . . . . 32 



\section{References}

Acehan, D., Malhotra, A., Xu, Y., Ren, M., Stokes, D. L., and Schlame, M. (2011). Cardiolipin affects the supramolecular organization of atp synthase in mitochondria. Biophys J, 100(9):2184-2192.

Alavian, K. N., Beutner, G., Lazrove, E., Sacchetti, S., Park, H. A., Licznerski, P., Li, H., Nabili, P., Hockensmith, K., Graham, M., Porter, G. A., J., and Jonas, E. A. (2014). An uncoupling channel within the c-subunit ring of the f1fo atp synthase is the mitochondrial permeability transition pore. Proc Natl Acad Sci U S A, 111(29):10580-10585.

Alkhaja, A. K., Jans, D. C., Nikolov, M., Vukotic, M., Lytovchenko, O., Ludewig, F., Schliebs, W., Riedel, D., Urlaub, H., Jakobs, S., and Deckers, M. (2012). Minos1 is a conserved component of mitofilin complexes and required for mitochondrial function and cristae organization. Mol Biol Cell, 23(2):247-257.

Allen, R. D. (1995). Membrane tubulation and proton pumps. Protoplasma, 189(1):1-8.

Allen, R. D., Schroeder, C. C., and Fok, A. K. (1989). An investigation of mitochondrial inner membranes by rapid-freeze deep-etch techniques. J Cell Biol, 108(6):2233-2240.

Amutha, B., Gordon, D. M., Gu, Y., and Pain, D. (2004). A novel role of mgm1p, a dynamin-related gtpase, in atp synthase assembly and cristae formation/maintenance. Biochem J, 381(Pt 1):19-23.

Anand, R., Wai, T., Baker, M. J., Kladt, N., Schauss, A. C., Rugarli, E., and Langer, T. (2014). The i-aaa protease yme1l and oma1 cleave opa1 to balance mitochondrial fusion and fission. J Cell Biol, 204(6):919-929.

Angelova, M. I. and Dimitrov, D. S. (1986). Liposome electroformation. Faraday Discuss. Chem. Soc., 81(0):303-311. 
Arnold, I., Pfeiffer, K., Neupert, W., Stuart, R. A., and Schagger, H. (1998). Yeast mitochondrial f1f0-atp synthase exists as a dimer: identification of three dimerspecific subunits. EMBO J, 17(24):7170-7178.

Arselin, G., Giraud, M. F., Dautant, A., Vaillier, J., Brethes, D., Coulary-Salin, B., Schaeffer, J., and Velours, J. (2003). The gxxxg motif of the transmembrane domain of subunit $e$ is involved in the dimerization/oligomerization of the yeast atp synthase complex in the mitochondrial membrane. Eur J Biochem, 270(8):1875-1884.

Baker, L. A., Watt, I. N., Runswick, M. J., Walker, J. E., and Rubinstein, J. L. (2012). Arrangement of subunits in intact mammalian mitochondrial atp synthase determined by cryo-em. Proc Natl Acad Sci U S A, 109(29):11675-11680.

Baker, M. J., Tatsuta, T., and Langer, T. (2011). Quality control of mitochondrial proteostasis. Cold Spring Harb Perspect Biol, 3(7):1-19.

Ban, T., Heymann, J. A., Song, Z., Hinshaw, J. E., and Chan, D. C. (2010). Opa1 disease alleles causing dominant optic atrophy have defects in cardiolipinstimulated gtp hydrolysis and membrane tubulation. Hum Mol Genet, 19(11):21132122.

Banci, L., Bertini, I., Ciofi-Baffoni, S., Janicka, A., Martinelli, M., Kozlowski, H., and Palumaa, P. (2008). A structural-dynamical characterization of human cox17. J Biol Chem, 283(12):7912-7920.

Barbot, M., Jans, D. C., Schulz, C., Denkert, N., Kroppen, B., Hoppert, M., Jakobs, S., and Meinecke, M. (2015). Mic10 oligomerizes to bend mitochondrial inner membranes at cristae junctions. Cell Metab, 21(5):756-763.

Bartlett, K. and Eaton, S. (2004). Mitochondrial beta-oxidation. Eur J Biochem, 271(3):462-469.

Bohnert, M., Pfanner, N., and van der Laan, M. (2015a). Mitochondrial machineries for insertion of membrane proteins. Curr Opin Struct Biol, 33:92-102.

Bohnert, M., Wenz, L. S., Zerbes, R. M., Horvath, S. E., Stroud, D. A., von der Malsburg, K., Muller, J. M., Oeljeklaus, S., Perschil, I., Warscheid, B., Chacinska, A., Veenhuis, M., van der Klei, I. J., Daum, G., Wiedemann, N., Becker, T., Pfanner, N., and van der Laan, M. (2012). Role of mitochondrial inner membrane organizing system in protein biogenesis of the mitochondrial outer membrane. Mol Biol Cell, 23(20):3948-3956. 
Bohnert, M., Zerbes, R. M., Davies, K. M., Muhleip, A. W., Rampelt, H., Horvath, S. E., Boenke, T., Kram, A., Perschil, I., Veenhuis, M., Kuhlbrandt, W., van der Klei, I. J., Pfanner, N., and van der Laan, M. (2015b). Central role of mic10 in the mitochondrial contact site and cristae organizing system. Cell Metab, 21(5):747755.

Bolanos-Garcia, V. M. and Miguel, R. N. (2003). On the structure and function of apolipoproteins: more than a family of lipid-binding proteins. Prog Biophys Mol Biol, 83(1):47-68.

Bornhovd, C., Vogel, F., Neupert, W., and Reichert, A. S. (2006). Mitochondrial membrane potential is dependent on the oligomeric state of f1f0-atp synthase supracomplexes. J Biol Chem, 281(20):13990-13998.

Boyle, G. M., Roucou, X., Nagley, P., Devenish, R. J., and Prescott, M. (1999). Identification of subunit $g$ of yeast mitochondrial $\mathrm{f} 1 \mathrm{fO}$-atp synthase, a protein required for maximal activity of cytochrome c oxidase. Eur J Biochem, 262(2):315-323.

Bustos, D. M. and Velours, J. (2005). The modification of the conserved gxxxg motif of the membrane-spanning segment of subunit $g$ destabilizes the supramolecular species of yeast atp synthase. J Biol Chem, 280(32):29004-29010.

Canham, P. B. (1970). The minimum energy of bending as a possible explanation of the biconcave shape of the human red blood cell. J Theor Biol, 26(1):61-81.

Cheng, W. C., Leach, K. M., and Hardwick, J. M. (2008). Mitochondrial death pathways in yeast and mammalian cells. Biochim Biophys Acta, 1783(7):12721279.

Cipolat, S., Martins de Brito, O., Dal Zilio, B., and Scorrano, L. (2004). Opa1 requires mitofusin 1 to promote mitochondrial fusion. Proc Natl Acad Sci U S A, 101(45):15927-15932.

Cipolat, S., Rudka, T., Hartmann, D., Costa, V., Serneels, L., Craessaerts, K., Metzger, K., Frezza, C., Annaert, W., D’Adamio, L., Derks, C., Dejaegere, T., Pellegrini, L., D'Hooge, R., Scorrano, L., and De Strooper, B. (2006). Mitochondrial rhomboid parl regulates cytochrome $\mathrm{c}$ release during apoptosis via opa1-dependent cristae remodeling. Cell, 126(1):163-175.

Civiletto, G., Varanita, T., Cerutti, R., Gorletta, T., Barbaro, S., Marchet, S., Lamperti, C., Viscomi, C., Scorrano, L., and Zeviani, M. (2015). Opa1 overexpres- 
sion ameliorates the phenotype of two mitochondrial disease mouse models. Cell Metab, 21(6):845-854.

Claypool, S. M. and Koehler, C. M. (2012). The complexity of cardiolipin in health and disease. Trends Biochem Sci, 37(1):32-41.

Daley, D. O., Adams, K. L., Clifton, R., Qualmann, S., Millar, A. H., Palmer, J. D., Pratje, E., and Whelan, J. (2002). Gene transfer from mitochondrion to nucleus: novel mechanisms for gene activation from cox2. Plant $J, 30(1): 11-21$.

Danino, D. and Hinshaw, J. E. (2001). Dynamin family of mechanoenzymes. Curr Opin Cell Biol, 13(4):454-460.

Darshi, M., Mendiola, V. L., Mackey, M. R., Murphy, A. N., Koller, A., Perkins, G. A., Ellisman, M. H., and Taylor, S. S. (2011). Chchd3, an inner mitochondrial membrane protein, is essential for maintaining crista integrity and mitochondrial function. J Biol Chem, 286(4):2918-2932.

Darshi, M., Trinh, K. N., Murphy, A. N., and Taylor, S. S. (2012). Targeting and import mechanism of coiled-coil helix coiled-coil helix domain-containing protein 3 (chchd3) into the mitochondrial intermembrane space. J Biol Chem, 287(47):39480-39491.

Davies, K. M., Anselmi, C., Wittig, I., Faraldo-Gomez, J. D., and Kuhlbrandt, W. (2012). Structure of the yeast f1fo-atp synthase dimer and its role in shaping the mitochondrial cristae. Proc Natl Acad Sci U S A, 109(34):13602-13607.

Davies, K. M., Strauss, M., Daum, B., Kief, J. H., Osiewacz, H. D., Rycovska, A., Zickermann, V., and Kuhlbrandt, W. (2011). Macromolecular organization of atp synthase and complex $\mathrm{i}$ in whole mitochondria. Proc Natl Acad Sci USA, 108(34):14121-14126.

Delettre, C., Griffoin, J. M., Kaplan, J., Dollfus, H., Lorenz, B., Faivre, L., Lenaers, G., Belenguer, P., and Hamel, C. P. (2001). Mutation spectrum and splicing variants in the opa1 gene. Hum Genet, 109(6):584-591.

Demishtein-Zohary, K., Marom, M., Neupert, W., Mokranjac, D., and Azem, A. (2015). Gxxxg motifs hold the tim23 complex together. FEBS J, 282(11):21782186. 
DeVay, R. M., Dominguez-Ramirez, L., Lackner, L. L., Hoppins, S., Stahlberg, H., and Nunnari, J. (2009). Coassembly of mgm1 isoforms requires cardiolipin and mediates mitochondrial inner membrane fusion. J Cell Biol, 186(6):793-803.

Ding, C., Wu, Z., Huang, L., Wang, Y., Xue, J., Chen, S., Deng, Z., Wang, L., Song, Z., and Chen, S. (2015). Mitofilin and chchd6 physically interact with sam50 to sustain cristae structure. Sci Rep, 5:1-11.

Dolezal, P., Likic, V., Tachezy, J., and Lithgow, T. (2006). Evolution of the molecular machines for protein import into mitochondria. Science, 313(5785):314-318.

Dudek, J., Rehling, P., and van der Laan, M. (2013). Mitochondrial protein import: Common principles and physiological networks. Biochim Biophys Acta, 1833(2):274-285.

Dyall, S. D., Brown, M. T., and Johnson, P. J. (2004). Ancient invasions: from endosymbionts to organelles. Science, 304(5668):253-257.

Eble, K. S., Coleman, W. B., Hantgan, R. R., and Cunningham, C. C. (1990). Tightly associated cardiolipin in the bovine heart mitochondrial atp synthase as analyzed by 31p nuclear magnetic resonance spectroscopy. J Biol Chem, 265(32):1943419440.

Erdmann, R., Veenhuis, M., Mertens, D., and Kunau, W. H. (1989). Isolation of peroxisome-deficient mutants of saccharomyces cerevisiae. Proc Natl Acad Sci U $S A, 86(14): 5419-5423$.

Esser, K., Tursun, B., Ingenhoven, M., Michaelis, G., and Pratje, E. (2002). A novel two-step mechanism for removal of a mitochondrial signal sequence involves the maaa complex and the putative rhomboid protease pcp1. J Mol Biol, 323(5):835843.

Evans, E. and Rawicz, W. (1990). Entropy-driven tension and bending elasticity in condensed-fluid membranes. Phys Rev Lett, 64(17):2094-2097.

Farsad, K., Ringstad, N., Takei, K., Floyd, S. R., Rose, K., and De Camilli, P. (2001). Generation of high curvature membranes mediated by direct endophilin bilayer interactions. J Cell Biol, 155(2):193-200.

Fawcett, D. W. (1981). Mitochondria, volume 2, pages 410-485. Philadelphia, 2 edition. 
Frey, T. G. and Mannella, C. A. (2000). The internal structure of mitochondria. Trends Biochem Sci, 25(7):319-324.

Frey, T. G., Renken, C. W., and Perkins, G. A. (2002). Insight into mitochondrial structure and function from electron tomography. Biochim Biophys Acta, 1555(13):196-203.

Frezza, C., Cipolat, S., Martins de Brito, O., Micaroni, M., Beznoussenko, G. V., Rudka, T., Bartoli, D., Polishuck, R. S., Danial, N. N., De Strooper, B., and Scorrano, L. (2006). Opa1 controls apoptotic cristae remodeling independently from mitochondrial fusion. Cell, 126(1):177-189.

Friedman, J. R., Mourier, A., Yamada, J., McCaffery, J. M., and Nunnari, J. (2015). Micos coordinates with respiratory complexes and lipids to establish mitochondrial inner membrane architecture. Elife, 4.

Gallop, J. L., Jao, C. C., Kent, H. M., Butler, P. J., Evans, P. R., Langen, R., and McMahon, H. T. (2006). Mechanism of endophilin n-bar domain-mediated membrane curvature. EMBO J, 25(12):2898-2910.

Galluzzi, L., Kepp, O., Trojel-Hansen, C., and Kroemer, G. (2012). Mitochondrial control of cellular life, stress, and death. Circ Res, 111(9):1198-1207.

Genin, E. C., Plutino, M., Bannwarth, S., Villa, E., Cisneros-Barroso, E., Roy, M., Ortega-Vila, B., Fragaki, K., Lespinasse, F., Pinero-Martos, E., Auge, G., Moore, D., Burte, F., Lacas-Gervais, S., Kageyama, Y., Itoh, K., Yu-Wai-Man, P., Sesaki, H., Ricci, J. E., Vives-Bauza, C., and Paquis-Flucklinger, V. (2015). Chchd10 mutations promote loss of mitochondrial cristae junctions with impaired mitochondrial genome maintenance and inhibition of apoptosis. EMBO Mol Med, 8(1):58-72.

Gieffers, C., Korioth, F., Heimann, P., Ungermann, C., and Frey, J. (1997). Mitofilin is a transmembrane protein of the inner mitochondrial membrane expressed as two isoforms. Exp Cell Res, 232(2):395-399.

Gietz, R. D. and Woods, R. A. (2002). Transformation of yeast by lithium acetate/single-stranded carrier dna/polyethylene glycol method. Methods Enzymol, 350:87-96.

Girard, P., Pecreaux, J., Lenoir, G., Falson, P., Rigaud, J. L., and Bassereau, P. (2004). A new method for the reconstitution of membrane proteins into giant unilamellar vesicles. Biophys J, 87(1):419-429. 
Giraud, M. F., Paumard, P., Soubannier, V., Vaillier, J., Arselin, G., Salin, B., Schaeffer, J., Brethes, D., di Rago, J. P., and Velours, J. (2002). Is there a relationship between the supramolecular organization of the mitochondrial atp synthase and the formation of cristae? Biochim Biophys Acta, 1555(1-3):174-180.

Guarani, V., McNeill, E. M., Paulo, J. A., Huttlin, E. L., Fröhlich, F., Gygi, S. P., Van Vactor, D., and Harper, J. W. (2015). Qil1 is a novel mitochondrial protein required for micos complex stability and cristae morphology. eLife, 4.

Hanahan, D. (1983). Studies on transformation of escherichia coli with plasmids. $J$ Mol Biol, 166(4):557-580.

Hardwick, J. M. and Cheng, W. C. (2004). Mitochondrial programmed cell death pathways in yeast. Dev Cell, 7(5):630-632.

Harner, M., Körner, C., Walther, D., Mokranjac, D., Kaesmacher, J., Welsch, U., Griffith, J., Mann, M., Reggiori, F., and Neupert, W. (2011). The mitochondrial contact site complex, a determinant of mitochondrial architecture. The EMBO Journal, 30(21):4356-4370.

Harner, M. E., Unger, A. K., Izawa, T., Walther, D. M., Ozbalci, C., Geimer, S., Reggiori, F., Brugger, B., Mann, M., Westermann, B., and Neupert, W. (2014). Aim24 and micos modulate respiratory function, tafazzin-related cardiolipin modification and mitochondrial architecture. Elife, 3.

Head, B. P., Zulaika, M., Ryazantsev, S., and van der Bliek, A. M. (2011). A novel mitochondrial outer membrane protein, moma-1, that affects cristae morphology in caenorhabditis elegans. Mol Biol Cell, 22(6):831-841.

Helfrich, W. (1973). Elastic properties of lipid bilayers: theory and possible experiments. Z Naturforsch C, 28(11):693-703.

Herlan, M., Vogel, F., Bornhovd, C., Neupert, W., and Reichert, A. S. (2003). Processing of mgm1 by the rhomboid-type protease pcp1 is required for maintenance of mitochondrial morphology and of mitochondrial dna. J Biol Chem, 278(30):27781-27788.

Herrmann, J. M. (2011). Minos is plus: a mitofilin complex for mitochondrial membrane contacts. Dev Cell, 21(4):599-600. 
Hill, K., Model, K., Ryan, M. T., Dietmeier, K., Martin, F., Wagner, R., and Pfanner, N. (1998). Tom40 forms the hydrophilic channel of the mitochondrial import pore for preproteins. Nature, 395(6701):516-521.

Hinnah, S. C., Hill, K., Wagner, R., Schlicher, T., and Soll, J. (1997). Reconstitution of a chloroplast protein import channel. EMBO J, 16(24):7351-7360.

Hoppins, S., Collins, S. R., Cassidy-Stone, A., Hummel, E., DeVay, R. M., Lackner, L. L., Westermann, B., Schuldiner, M., Weissman, J. S., and Nunnari, J. (2011). A mitochondrial-focused genetic interaction map reveals a scaffold-like complex required for inner membrane organization in mitochondria. J Cell Biol, 195(2):323340.

Horvath, S. E., Rampelt, H., Oeljeklaus, S., Warscheid, B., van der Laan, M., and Pfanner, N. (2015). Role of membrane contact sites in protein import into mitochondria. Protein Sci, 24(3):277-297.

Hu, J., Shibata, Y., Voss, C., Shemesh, T., Li, Z., Coughlin, M., Kozlov, M. M., Rapoport, T. A., and Prinz, W. A. (2008). Membrane proteins of the endoplasmic reticulum induce high-curvature tubules. Science, 319(5867):1247-1250.

Huang, K. C. and Ramamurthi, K. S. (2010). Macromolecules that prefer their membranes curvy. Mol Microbiol, 76(4):822-832.

Huh, W. K., Falvo, J. V., Gerke, L. C., Carroll, A. S., Howson, R. W., Weissman, J. S., and O'Shea, E. K. (2003). Global analysis of protein localization in budding yeast. Nature, 425(6959):686-691.

Huynen, M. A., Muhlmeister, M., Gotthardt, K., Guerrero-Castillo, S., and Brandt, U. (2016). Evolution and structural organization of the mitochondrial contact site (micos) complex and the mitochondrial intermembrane space bridging (mib) complex. Biochim Biophys Acta, 1863(1):91-101.

Jans, D. C., Wurm, C. A., Riedel, D., Wenzel, D., Stagge, F., Deckers, M., Rehling, P., and Jakobs, S. (2013). Sted super-resolution microscopy reveals an array of minos clusters along human mitochondria. Proc Natl Acad Sci U S A, 110(22):89368941.

Jiko, C., Davies, K. M., Shinzawa-Itoh, K., Tani, K., Maeda, S., Mills, D. J., Tsukihara, T., Fujiyoshi, Y., Kuhlbrandt, W., and Gerle, C. (2015). Bovine f1fo atp synthase monomers bend the lipid bilayer in $2 d$ membrane crystals. Elife, 4. 
John, G. B., Shang, Y., Li, L., Renken, C., Mannella, C. A., Selker, J. M. L., Rangell, L., Bennett, M. J., and Zha, J. (2005). The mitochondrial inner membrane protein mitofilin controls cristae morphology. Mol Biol Cell, 16(3):1543-1554.

Koob, S., Barrera, M., Anand, R., and Reichert, A. S. (2015). The non-glycosylated isoform of mic26 is a constituent of the mammalian micos complex and promotes formation of crista junctions. Biochim Biophys Acta, 1853(7):1551-1563.

Koob, S. and Reichert, A. S. (2014). Novel intracellular functions of apolipoproteins: the apoo protein family as constituents of the mitofilin/minos complex determines cristae morphology in mitochondria. Biol Chem, 395(3):285-296.

Kovermann, P., Truscott, K. N., Guiard, B., Rehling, P., Sepuri, N. B., Müller, H., Jensen, R. E., Wagner, R., and Pfanner, N. (2002). Tim22, the essential core of the mitochondrial protein insertion complex, forms a voltage-activated and signal-gated channel. Mol Cell, 9(2):363-373.

Kozlov, M. M., Campelo, F., Liska, N., Chernomordik, L. V., Marrink, S. J., and McMahon, H. T. (2014). Mechanisms shaping cell membranes. Curr Opin Cell Biol, 29:53-60.

Krebs, J. J., Hauser, H., and Carafoli, E. (1979). Asymmetric distribution of phospholipids in the inner membrane of beef heart mitochondria. J Biol Chem, 254(12):5308-5316.

Körner, C., Barrera, M., Dukanovic, J., Eydt, K., Harner, M., Rabl, R., Vogel, F., Rapaport, D., Neupert, W., and Reichert, A. S. (2012). The c-terminal domain of fcj1 is required for formation of crista junctions and interacts with the tob/sam complex in mitochondria. Mol Biol Cell, 23(11):2143-2155.

Kuhlbrandt, W. (2015). Structure and function of mitochondrial membrane protein complexes. BMC Biol, 13(89).

Labbe, K., Murley, A., and Nunnari, J. (2014). Determinants and functions of mitochondrial behavior. Annu Rev Cell Dev Biol, 30:357-391.

Lamant, M., Smih, F., Harmancey, R., Philip-Couderc, P., Pathak, A., Roncalli, J., Galinier, M., Collet, X., Massabuau, P., Senard, J. M., and Rouet, P. (2006). Apoo, a novel apolipoprotein, is an original glycoprotein up-regulated by diabetes in human heart. J Biol Chem, 281(47):36289-36302. 
Li, H., Ruan, Y., Zhang, K., Jian, F., Hu, C., Miao, L., Gong, L., Sun, L., Zhang, X., Chen, S., Chen, H., Liu, D., and Song, Z. (2015). Mic60/mitofilin determines micos assembly essential for mitochondrial dynamics and mtdna nucleoid organization. Cell Death Differ, 23(3):380-392.

Lill, R., Hoffmann, B., Molik, S., Pierik, A. J., Rietzschel, N., Stehling, O., Uzarska, M. A., Webert, H., Wilbrecht, C., and Muhlenhoff, U. (2012). The role of mitochondria in cellular iron-sulfur protein biogenesis and iron metabolism. Biochim Biophys Acta, 1823(9):1491-1508.

Mannella, C. A. (2006). The relevance of mitochondrial membrane topology to mitochondrial function. Biochim Biophys Acta, 1762(2):140-147.

Mannella, C. A., Marko, M., Penczek, P., Barnard, D., and Frank, J. (1994). The internal compartmentation of rat-liver mitochondria: tomographic study using the high-voltage transmission electron microscope. Microsc Res Tech, 27(4):278-283. McMahon, H. T. and Boucrot, E. (2011). Molecular mechanism and physiological functions of clathrin-mediated endocytosis. Nat Rev Mol Cell Biol, 12(8):517-533.

Meeusen, S., DeVay, R., Block, J., Cassidy-Stone, A., Wayson, S., McCaffery, J. M., and Nunnari, J. (2006). Mitochondrial inner-membrane fusion and crista maintenance requires the dynamin-related gtpase mgm1. Cell, 127(2):383-395.

Meinecke, M., Boucrot, E., Camdere, G., Hon, W. C., Mittal, R., and McMahon, H. T. (2013). Cooperative recruitment of dynamin and bin/amphiphysin/rvs (bar) domain-containing proteins leads to gtp-dependent membrane scission. $J$ Biol Chem, 288(9):6651-6661.

Meinecke, M., Wagner, R., Kovermann, P., Guiard, B., Mick, D. U., Hutu, D. P., Voos, W., Truscott, K. N., Chacinska, A., Pfanner, N., and Rehling, P. (2006). Tim50 maintains the permeability barrier of the mitochondrial inner membrane. Science, 312(5779):1523-1526.

Meisinger, C., Pfanner, N., and Truscott, K. N. (2006). Isolation of yeast mitochondria. Methods Mol Biol, 313:33-39.

Merkwirth, C., Dargazanli, S., Tatsuta, T., Geimer, S., Lower, B., Wunderlich, F. T., von Kleist-Retzow, J. C., Waisman, A., Westermann, B., and Langer, T. (2008). Prohibitins control cell proliferation and apoptosis by regulating opa1-dependent cristae morphogenesis in mitochondria. Genes Dev, 22(4):476-488. 
Merkwirth, C. and Langer, T. (2009). Prohibitin function within mitochondria: essential roles for cell proliferation and cristae morphogenesis. Biochim Biophys Acta, 1793(1):27-32.

Mileykovskaya, E. and Dowhan, W. (2000). Visualization of phospholipid domains in escherichia coli by using the cardiolipin-specific fluorescent dye 10-n-nonyl acridine orange. J Bacteriol, 182(4):1172-1175.

Muench, S. P., Trinick, J., and Harrison, M. A. (2011). Structural divergence of the rotary atpases. $Q$ Rev Biophys, 44(3):311-356.

Mun, J. Y., Lee, T. H., Kim, J. H., Yoo, B. H., Bahk, Y. Y., Koo, H. S., and Han, S. S. (2010). Caenorhabditis elegans mitofilin homologs control the morphology of mitochondrial cristae and influence reproduction and physiology. J Cell Physiol, 224(3):748-756.

Munoz-Gomez, S. A., Slamovits, C. H., Dacks, J. B., Baier, K. A., Spencer, K. D., and Wideman, J. G. (2015). Ancient homology of the mitochondrial contact site and cristae organizing system points to an endosymbiotic origin of mitochondrial cristae. Curr Biol, 25(11):1489-1495.

Neupert, W. (2012). Snapshot: Mitochondrial architecture. Cell, 149(3):722.

Neupert, W. and Herrmann, J. M. (2007). Translocation of proteins into mitochondria. Annu Rev Biochem, 76:723-749.

Nishibori, A., Kusaka, J., Hara, H., Umeda, M., and Matsumoto, K. (2005). Phosphatidylethanolamine domains and localization of phospholipid synthases in bacillus subtilis membranes. J Bacteriol, 187(6):2163-2174.

Odgren, P. R., Toukatly, G., Bangs, P. L., Gilmore, R., and Fey, E. G. (1996). Molecular characterization of mitofilin (hmp), a mitochondria-associated protein with predicted coiled coil and intermembrane space targeting domains. J Cell Sci, 109 ( Pt 9):2253-2264.

Olichon, A., Baricault, L., Gas, N., Guillou, E., Valette, A., Belenguer, P., and Lenaers, G. (2003). Loss of opa1 perturbates the mitochondrial inner membrane structure and integrity, leading to cytochrome c release and apoptosis. J Biol Chem, 278(10):7743-7746. 
Olichon, A., Emorine, L. J., Descoins, E., Pelloquin, L., Brichese, L., Gas, N., Guillou, E., Delettre, C., Valette, A., Hamel, C. P., Ducommun, B., Lenaers, G., and Belenguer, P. (2002). The human dynamin-related protein opa1 is anchored to the mitochondrial inner membrane facing the inter-membrane space. FEBS Lett, 523(1-3):171-176.

Ortiz, A., Killian, J. A., Verkleij, A. J., and Wilschut, J. (1999). Membrane fusion and the lamellar-to-inverted-hexagonal phase transition in cardiolipin vesicle systems induced by divalent cations. Biophys J, 77(4):2003-2014.

Osman, C., Merkwirth, C., and Langer, T. (2009). Prohibitins and the functional compartmentalization of mitochondrial membranes. J Cell Sci, 122(Pt 21):38233830 .

Ostermeyer, A. G., Ramcharan, L. T., Zeng, Y., Lublin, D. M., and Brown, D. A. (2004). Role of the hydrophobic domain in targeting caveolin-1 to lipid droplets. $J$ Cell Biol, 164(1):69-78.

Ott, C., Dorsch, E., Fraunholz, M., Straub, S., and Kozjak-Pavlovic, V. (2015). Detailed analysis of the human mitochondrial contact site complex indicate a hierarchy of subunits. PLoS One, 10(3):1-15.

Ott, C., Ross, K., Straub, S., Thiede, B., Gotz, M., Goosmann, C., Krischke, M., Mueller, M. J., Krohne, G., Rudel, T., and Kozjak-Pavlovic, V. (2012). Sam50 functions in mitochondrial intermembrane space bridging and biogenesis of respiratory complexes. Mol Cell Biol, 32(6):1173-1188.

Paumard, P., Vaillier, J., Coulary, B., Schaeffer, J., Soubannier, V., Mueller, D. M., Brethes, D., di Rago, J. P., and Velours, J. (2002). The atp synthase is involved in generating mitochondrial cristae morphology. EMBO J, 21(3):221-230.

Pellegrini, L. and Scorrano, L. (2007). A cut short to death: Parl and opa1 in the regulation of mitochondrial morphology and apoptosis. Cell Death Differ, 14(7):12751284.

Perkins, G., Goodenough, D., and Sosinsky, G. (1997). Three-dimensional structure of the gap junction connexon. Biophys J, 72(2 Pt 1):533-544.

Perkins, G. A., Scott, R., Perez, A., Ellisman, M. H., Johnson, J. E., and Fox, D. A. (2012). Bcl-xl-mediated remodeling of rod and cone synaptic mitochondria after 
postnatal lead exposure: electron microscopy, tomography and oxygen consumption. Mol Vis, 18:3029-3048.

Pfanner, N., van der Laan, M., Amati, P., Capaldi, R. A., Caudy, A. A., Chacinska, A., Darshi, M., Deckers, M., Hoppins, S., Icho, T., Jakobs, S., Ji, J., Kozjak-Pavlovic, V., Meisinger, C., Odgren, P. R., Park, S. K., Rehling, P., Reichert, A. S., Sheikh, M. S., Taylor, S. S., Tsuchida, N., van der Bliek, A. M., van der Klei, I. J., Weissman, J. S., Westermann, B., Zha, J., Neupert, W., and Nunnari, J. (2014). Uniform nomenclature for the mitochondrial contact site and cristae organizing system. $J$ Cell Biol, 204(7):1083-1086.

Rabl, R., Soubannier, V., Scholz, R., Vogel, F., Mendl, N., Vasiljev-Neumeyer, A., Körner, C., Jagasia, R., Keil, T., Baumeister, W., Cyrklaff, M., Neupert, W., and Reichert, A. S. (2009). Formation of cristae and crista junctions in mitochondria depends on antagonism between fcj1 and su e/g. J Cell Biol, 185(6):1047-1063.

Rizzuto, R., De Stefani, D., Raffaello, A., and Mammucari, C. (2012). Mitochondria as sensors and regulators of calcium signalling. Nat Rev Mol Cell Biol, 13(9):566578.

Rujiviphat, J., Wong, M. K., Won, A., Shih, Y.-I., Yip, C. M., and McQuibban, G. A. (2015). Mitochondrial genome maintenance 1 (mgm1) protein alters membrane topology and promotes local membrane bending. J Mol Biol, 427(16):2599-2609.

Russ, W. P. and Engelman, D. M. (2000). The gxxxg motif: a framework for transmembrane helix-helix association. J Mol Biol, 296(3):911-919.

Sackmann, E. (2014). Endoplasmatic reticulum shaping by generic mechanisms and protein-induced spontaneous curvature. Adv Colloid Interface Sci, 208:153160.

Sakowska, P., Jans, D. C., Mohanraj, K., Riedel, D., Jakobs, S., and Chacinska, A. (2015). The oxidation status of mic19 regulates micos assembly. Mol Cell Biol, 35(24):4222-4237.

Schagger, H., de Coo, R., Bauer, M. F., Hofmann, S., Godinot, C., and Brandt, U. (2004). Significance of respirasomes for the assembly/stability of human respiratory chain complex i. J Biol Chem, 279(35):36349-36353.

Schauble, S., King, C. C., Darshi, M., Koller, A., Shah, K., and Taylor, S. S. (2007). Identification of chchd3 as a novel substrate of the camp-dependent protein kinase 
(pka) using an analog-sensitive catalytic subunit. J Biol Chem, 282(20):1495214959.

Schlame, M. (2013). Cardiolipin remodeling and the function of tafazzin. Biochim Biophys Acta, 1831(3):582-588.

Scorrano, L., Ashiya, M., Buttle, K., Weiler, S., Oakes, S. A., Mannella, C. A., and Korsmeyer, S. J. (2002). A distinct pathway remodels mitochondrial cristae and mobilizes cytochrome c during apoptosis. Dev Cell, 2(1):55-67.

Sesaki, H., Southard, S. M., Yaffe, M. P., and Jensen, R. E. (2003). Mgm1p, a dynamin-related gtpase, is essential for fusion of the mitochondrial outer membrane. Mol Biol Cell, 14(6):2342-2356.

Sherman, F. (2002). Getting started with yeast. Methods Enzymol, 350:3-41.

Shibata, Y., Hu, J., Kozlov, M. M., and Rapoport, T. A. (2009). Mechanisms shaping the membranes of cellular organelles. Annu Rev Cell Dev Biol, 25:329-354.

Sikorski, R. S. and Hieter, P. (1989). A system of shuttle vectors and yeast host strains designed for efficient manipulation of dna in saccharomyces cerevisiae. Genetics, 122(1):19-27.

Stachowiak, J. C., Brodsky, F. M., and Miller, E. A. (2013). A cost-benefit analysis of the physical mechanisms of membrane curvature. Nat Cell Biol, 15(9):1019-1027.

Stachowiak, J. C., Schmid, E. M., Ryan, C. J., Ann, H. S., Sasaki, D. Y., Sherman, M. B., Geissler, P. L., Fletcher, D. A., and Hayden, C. C. (2012). Membrane bending by protein-protein crowding. Nat Cell Biol, 14(9):944-949.

Stoldt, S., Wenzel, D., Hildenbeutel, M., Wurm, C. A., Herrmann, J. M., and Jakobs, S. (2012). The inner-mitochondrial distribution of oxa1 depends on the growth conditions and on the availability of substrates. Mol Biol Cell, 23(12):2292-2301.

Strauss, M., Hofhaus, G., Schroder, R. R., and Kuhlbrandt, W. (2008). Dimer ribbons of atp synthase shape the inner mitochondrial membrane. EMBO J, 27(7):1154-1160.

Truscott, K. N., Kovermann, P., Geissler, A., Merlin, A., Meijer, M., Driessen, A. J., Rassow, J., Pfanner, N., and Wagner, R. (2001). A presequence- and voltagesensitive channel of the mitochondrial preprotein translocase formed by tim23. Nat Struct Biol, 8(12):1074-1082. 
van der Laan, M., Bohnert, M., Wiedemann, N., and Pfanner, N. (2012). Role of minos in mitochondrial membrane architecture and biogenesis. Trends Cell Biol, 22(4):185-192.

van der Laan, M., Meinecke, M., Dudek, J., Hutu, D. P., Lind, M., Perschil, I., Guiard, B., Wagner, R., Pfanner, N., and Rehling, P. (2007). Motor-free mitochondrial presequence translocase drives membrane integration of preproteins. Nat Cell Biol, 9(10):1152-1159.

Varabyova, A., Topf, U., Kwiatkowska, P., Wrobel, L., Kaus-Drobek, M., and Chacinska, A. (2013). Mia40 and minos act in parallel with ccs1 in the biogenesis of mitochondrial sod1. FEBS J, 280(20):4943-4959.

Varanita, T., Soriano, M. E., Romanello, V., Zaglia, T., Quintana-Cabrera, R., Semenzato, M., Menabo, R., Costa, V., Civiletto, G., Pesce, P., Viscomi, C., Zeviani, M., Di Lisa, F., Mongillo, M., Sandri, M., and Scorrano, L. (2015). The opa1dependent mitochondrial cristae remodeling pathway controls atrophic, apoptotic, and ischemic tissue damage. Cell Metab, 21(6):834-844.

Voeltz, G. K., Prinz, W. A., Shibata, Y., Rist, J. M., and Rapoport, T. A. (2006). A class of membrane proteins shaping the tubular endoplasmic reticulum. Cell, 124(3):573-586.

Vogel, F., Bornhövd, C., Neupert, W., and Reichert, A. S. (2006). Dynamic subcompartmentalization of the mitochondrial inner membrane. J Cell Biol, 175(2):237247.

von der Malsburg, K., Müller, J. M., Bohnert, M., Oeljeklaus, S., Kwiatkowska, P., Becker, T., Loniewska-Lwowska, A., Wiese, S., Rao, S., Milenkovic, D., Hutu, D. P., Zerbes, R. M., Schulze-Specking, A., Meyer, H. E., Martinou, J.-C., Rospert, S., Rehling, P., Meisinger, C., Veenhuis, M., Warscheid, B., van der Klei, I. J., Pfanner, N., Chacinska, A., and van der Laan, M. (2011). Dual role of mitofilin in mitochondrial membrane organization and protein biogenesis. Dev Cell, 21(4):694-707.

Vonck, J., von Nidda, T. K., Meier, T., Matthey, U., Mills, D. J., Kuhlbrandt, W., and Dimroth, P. (2002). Molecular architecture of the undecameric rotor of a bacterial na+-atp synthase. J Mol Biol, 321(2):307-316. 
Wagner, K., Perschil, I., Fichter, C. D., and van der Laan, M. (2010). Stepwise assembly of dimeric $f(1) f(0)$-atp synthase in mitochondria involves the small $f(0)$ subunits k and i. Mol Biol Cell, 21(9):1494-1504.

Wagner, K., Rehling, P., Sanjuan Szklarz, L. K., Taylor, R. D., Pfanner, N., and van der Laan, M. (2009). Mitochondrial f1fo-atp synthase: the small subunits e and $\mathrm{g}$ associate with monomeric complexes to trigger dimerization. $\mathrm{J} \mathrm{Mol} \mathrm{Biol,}$ 392(4):855-861.

Walker, J. E. (2013). The atp synthase: the understood, the uncertain and the unknown. Biochem Soc Trans, 41(1):1-16.

Webb, C. T., Gorman, M. A., Lazarou, M., Ryan, M. T., and Gulbis, J. M. (2006). Crystal structure of the mitochondrial chaperone tim9.10 reveals a six-bladed alpha-propeller. Mol Cell, 21(1):123-133.

Weber, T. A., Koob, S., Heide, H., Wittig, I., Head, B., van der Bliek, A., Brandt, U., Mittelbronn, M., and Reichert, A. S. (2013). Apool is a cardiolipin-binding constituent of the mitofilin/minos protein complex determining cristae morphology in mammalian mitochondria. PLOS ONE, 8(5):e63683.

Westermann, B. (2010). Mitochondrial fusion and fission in cell life and death. Nat Rev Mol Cell Biol, 11(12):872-884.

Williams, R. J. (2000). Mitochondria and chloroplasts: localized and delocalized bioenergetic transduction. Trends Biochem Sci, 25(10):479.

Wong, E. D., Wagner, J. A., Scott, S. V., Okreglak, V., Holewinske, T. J., CassidyStone, A., and Nunnari, J. (2003). The intramitochondrial dynamin-related gtpase, mgm1p, is a component of a protein complex that mediates mitochondrial fusion. $J$ Cell Biol, 160(3):303-311.

Wrede, C., Walbaum, U., Ducki, A., Heieren, I., and Hoppert, M. (2013). Localization of methyl-coenzyme $\mathrm{m}$ reductase as metabolic marker for diverse methanogenic archaea. Archaea, 2013:920241.

Wurm, C. A. and Jakobs, S. (2006). Differential protein distributions define two sub-compartments of the mitochondrial inner membrane in yeast. FEBS Letters, 580(24):5628-5634. 
Xie, J., Marusich, M. F., Souda, P., Whitelegge, J., and Capaldi, R. A. (2007). The mitochondrial inner membrane protein mitofilin exists as a complex with sam50, metaxins 1 and 2, coiled-coil-helix coiled-coil-helix domain-containing protein 3 and 6 and dnajc11. FEBS Lett, 581(18):3545-3549.

Zerbes, R. M., Bohnert, M., Stroud, D. A., von der Malsburg, K., Kram, A., Oeljeklaus, S., Warscheid, B., Becker, T., Wiedemann, N., Veenhuis, M., van der Klei, I. J., Pfanner, N., and van der Laan, M. (2012a). Role of minos in mitochondrial membrane architecture: cristae morphology and outer membrane interactions differentially depend on mitofilin domains. J Mol Biol, 422(2):183-191.

Zerbes, R. M., Hoss, P., Pfanner, N., van der Laan, M., and Bohnert, M. (2016). Distinct roles of mic12 and mic27 in the mitochondrial contact site and cristae organizing system. J Mol Biol, page 10.1016/j.jmb.2016.02.031.

Zerbes, R. M., van der Klei, I. J., Veenhuis, M., Pfanner, N., van der Laan, M., and Bohnert, M. (2012b). Mitofilin complexes: conserved organizers of mitochondrial membrane architecture. Biol Chem, 393(11):1247-1261.

Zick, M., Duvezin-Caubet, S., Schafer, A., Vogel, F., Neupert, W., and Reichert, A. S. (2009a). Distinct roles of the two isoforms of the dynamin-like gtpase mgm1 in mitochondrial fusion. FEBS Lett, 583(13):2237-2243.

Zick, M., Rabl, R., and Reichert, A. S. (2009b). Cristae formation-linking ultrastructure and function of mitochondria. Biochim Biophys Acta, 1793(1):5-19.

Zimmerberg, J. and Kozlov, M. M. (2006). How proteins produce cellular membrane curvature. Nat Rev Mol Cell Biol, 7(1):9-19.

Zinser, E., Sperka-Gottlieb, C. D., Fasch, E. V., Kohlwein, S. D., Paltauf, F., and Daum, G. (1991). Phospholipid synthesis and lipid composition of subcellular membranes in the unicellular eukaryote saccharomyces cerevisiae. J Bacteriol, 173(6):2026-2034. 



\section{Acknowledgments}

First of all I would like to thank my supervisor Prof. Dr. Michael Meinecke for giving me the opportunity to work on this exciting and challenging project, for his outstanding guidance, support and encouragement. Thanks for many fruitful scientific discussions and a pleasant working atmosphere throughout these four years.

There are a number of other people I would like to thank ...

... Prof. Dr. Stefan Jakobs and Prof. Dr. Tobias Moser for being members of my thesis committee and for their helpful comments during thesis committee meetings. Additionally, I would like to thank Prof. Dr. Stefan Jakobs for the collaborative work on this project.

... Dr. Daniel C. Jans for providing the electron microscopy pictures of yeast mitochondria.

... Dr. Christian Schulz for his contributions in yeast biochemical and molecular biology assays, for many important and valuable comments and suggestions.

... Niels Denkert for the FRET measurements and for many interesting scientific discussions.

... Benjamin Kroppen for the EM control experiments and for his support during the paper revision.

... Dr. Michael Hoppert who introduced me to electron microscopy and spent a great deal of time with me in front of the instrument.

... Katharina Krohn for her help and support in purifying proteins from 30 liter bacterial culture.

... Dr. Miriam Grace, Dr. Sylvie Callegari, Daryna Tarasenko and Dr. David Pacheu Grau for their helpful comments and suggestions on this thesis as well as for proof-reading it.

... Klaus Neifer for refreshing my knowledge about the HPLC and for his help in protein purification at the initial steps of this work.

... Göttingen graduate school GGNB.

Finally, I would like to thank ...

... my friends for their love and support.

... my German-French family, especially Steffi and Claude. Danke schön für eure Liebe und Unterstützung! Merci beaucoup! 
... my brother Ivane, his lovely wife Anna and their adorable daughters, Barbare and Lizi.

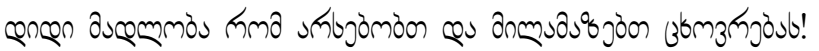

... my parents Tina and Jimi. Without their support, trust, help and love I would not have made it this far.

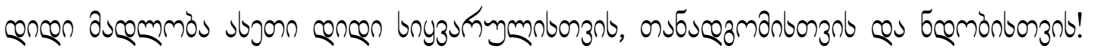

... my husband, Yann. Danke schön für deine uneingeschränkte Liebe, deine Unterstützung und dein Verständnis, besonderes in den letzten Monaten meiner Doktorarbeit. Ich bin sehr glücklich dich in meinem Leben zu haben! 